NUREG-0383

Volume 3

Revision 16

\title{
Directory of \\ Certificates of Compliance for \\ Radioactive Materials Packages
}

Report of NRC Approved Quality Assurance Programs for Radioactive Materials Packages

Manuscript Completed: October 1996

Date Published: October 1996

Spent Fuel Project Office

Office of Nuclear Material Safety and Safeguards

U.S. Nuclear Regulatory Commission

Washington, DC 20555-0001

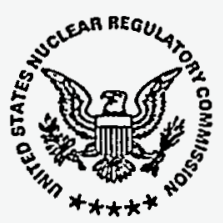




\section{DISCLAIMER}

Portions of this document may be illegible in electronic image products. Images are produced from the best available original document. 


\section{DISCLAIMER}

This report was prepared as an account of work sponsored by an agency of the United States Government. Neither the United States Government nor any agency thereof, nor any of their employees, makes any warranty, express or implied, or assumes any legal liability or responsibility for the accuracy, completeness, or usefulness of any information, apparatus, product, or process disclosed, or represents that its use would not infringe privately owned rights. Reference herein to any specific commercial product, process, or service by trade name, trademark, manufacturer, or otherwise does not necessarily constitute or imply its endorsement, recommendation, or favoring by the United States Government or any agency thereof. The views and opinions of authors expressed herein do not necessarily state or reflect those of the United States Government or any agency thereof. 


\section{FOREWORD}

The purpose of this directory is to make available a convenient source of information on packagings approved by the U.S. Nuclear Regulatory Commission. To assist in identifying packaging, an index by Model Number and corresponding Certificate of Compliance Number is included at the front of Volumes 1 and 2 . An alphabetical listing by user name is included in the back of Volume 3 for approved Quality Assurance programs. The reports include a 1 isting of al1 users of each package design and approved Quality Assurance programs prior to the publication date of the directory.

Comments to make future revisions of this directory more useful are invited and should be directed to the Spent Fuel Project Office, U.S. Nuclear Regulatory Commission. 
DOCKETH: 07100001

USER NAME: ANEFCO, INC,

MOODUS

ATTN: MR. JOHN D. MURPHY
REVISION\# : 4

CT 06469
EXPIRATION DATE: $03 / 31 / 2000$
ORIG. ISSUE DATE: $10 / 15 / 1979$

APPROVAL TYPE: NORMAL AND SPECIAL FORM

REGULATION: SUBPART H TO 10 CFR PART 71
USE(S)

DESIGN

FABRICATION

ASSEMBLY

TESTING

PROCUREMENT

MAINTENANCE

MODIFICATION

USE

COMMENTS:

DOCKETH : 07100003

REVISION\# : 13

USER NAME: SIEMENS POWER CORPORATION 2101 HORN RAPIDS ROAD, P. O. BOX 130 RICHLAND ATTN: MR. JAMES B. EDGAR
WA 993520130
EXPIRATION DATE: $11 / 30 / 1999$
ORIG. ISSUE DATE : $06 / 13 / 1979$

APPROVAL TYPE: NORMAL AND SPECIAL FORM

REGULATION: SUBPART H TO 10 CFR PART 71
$\operatorname{USE}(S)$

DESIGN

FABRICATION

ASSEMBLY

PROCUREMENT

MAINTENANCE

REPAIR

MOD IFICATION

USE

\section{COMMENTS :}

DOCKETH : 07100004

REVISION\# : 7

USER NAME: GENERAL ELECTRIC COMPANY, MORRIS OPERATION 7555 EAST COLLINS ROAD

MORRIS

ATTN: MR. L. L. DENIO
IL 604509740
EXPIRATION DATE: $05 / 31 / 2000$
ORIG. I SSUE DATE: $10 / 05 / 1979$

APPROVAL TYPE: SPECIAL FORM

REGULATION: APPENDIX B TO 10 CFR PART 50
USE(S)

PROCUREMENT MAINTENANCE REPAIR USE

COMMENTS : 
EXPIRATION DATE: $08 / 31 / 1999$

APPROVAL TYPE: NORMAL AND SPECIAL FORM

COMMONWEALTH EDISON
1400 OPUS PLACE, SUITE 500

DOWNERS GROVE

ATTN: MS. MARY M. VINCENT
IL 60515 REGULATION: APPENDIX B TO 10 CFR PART 50
FEE CATEGORY: $1 O B(2)$

\section{REVISION\#: 4} USER NAME: ARROW TANK \& ENGINEERING CO.
$650 \mathrm{~N}$. EMERSON

CAMBRIDGE

ATTN: MRS. LYNN HANSON
MN 55008

COMMENTS :

DOCKET\#: 07100015

REVISION\#: 6

USER NAME: CHICAGO BRIDGE \& IRON COMPANY 8900 FAIRBANKS NORTH HOUSTON ROAD HOUSTON TX 77064

ATTN: MR. HUGH K. HOWERTON
USE (S)

PROCUREMENT MAINTENANCE REPAIR
EXP IRATION DATE: 09/30/1999
ORIG: I SSUE DATE: $06 / 11 / 1979$

APPROVAL TYPE: SPECIAL FORM

REGULATION: SUBPART H TO 10 CFR PART 71 FEE CATEGORY: $10 B(2)$
USE (S)

PROCUREMENT MAINTENANCE REPAIR USE

COMMENTS :

EXPIRATION DATE: 08/31/1999

ORIG. ISSUE DATE: 06/29/1979

APPROVAL TYPE: SPECIAL FORM

REGULATION: SUBPART H TO 10 CFR PART 71

\section{$\operatorname{USE}(S)$}

PROCUREMENT MAINTENANCE MAINTENAN
REPAIR USE 
DOCKET\# : 07100016

REVISION\# : 4

USER NAME: NORTHWEST AIRLINES

5101 NORTHWEST DRIVE

$M / S$ C8B40

ST. PAUL JEFF A. REGISTER MN 551113034

COMMENTS :

DOCKETH : 07100017

REVISION\# : 4

USER NAME: RHODE ISLAND ATOMIC ENERGY COMMISSION NUCLEAR SCIENCE CENTER SOUTH FERRY ROAD ATTN: MR. WAYNE SIMONEAU

RI 028821197
EXP IRATION DATE: $09 / 30 / 1999$

APPROVAL TYPE: SPECIAL FORM REGULATION: SUBPART H TO 10 CFR PART 71
FEE CATEGORY: $10 B(2)$
USE(S)

PROCUREMENT

MAINTENANCE

REPAIR

USE

\section{COMMENTS :}

DOCKETH : 07100018

REVISION\# : 5

USER NAME: NAC INTERNATIONAL, INC. 655 ENGINEERING DRIVE SUITE 200 NORCROSS GARY TJERSLAND

GA 30092

NAC INTERNATIONAL, INC. 655 ENGINEERING DŔIVE SUITE 200 NORCROSS ATTN: MR. JOHN J. STOBBS

GA 30092
EXPIRATION DATE: $02 / 28 / 2001$

ORIG. ISSUE DATE: 08/29/1980

APPROVAL TYPE: SPECIAL FORM

REGULATION: SUBPART H TO 10 CFR PART 71
USE(S)

PROCUREMENT MAINTENANCE REPAIR
EXPIRATION DATE: $12 / 31 / 1999$

APPROVAL TYPE: NORMAL AND SPECIAL FORM

REGULATION: SUBPART H TO 10 CFR PART 71 FEE CATEGORY: $10 B(1)$
USE(S)

DESIGN FABR ICATION ASSEMBLY TESTING PRO MAINTEMENT REPAIR MOD IF ICATION

COMMENTS : 
DOCKETH : 07100019

REVISION\#: 6 USER NAME: $\begin{aligned} & \text { ROCHESTER GAS \& ELECTRIC CORP., GINNA STATION } \\ & \text { 89 EAST AVENUE }\end{aligned}$

ROCHESTER

ATTN: MR. R. C. MECREDY

NY 146490001

\section{COMMENTS :}

\section{DOCKETH : 07100023}

REVISION\# : 4

USER NAME: TENNESSEE GAS PIPELINE COMPANY 5510 SOUTH RICE AVE.

HOUSTON
ATTN: MR. DAVID L. CULBERTSON
77081

COMMENTS :

DOCKETH : 07100025

REVISION\# : 5

ROUTE 1, BOX 275
USER NAME: DAIRYLAND POWER COOPERATIVE
EXPIRATION DATE: 06/30/1999

APPROVAL TYPE: NORMAL AND SPECIAL FORM

REGULATION: APPENDIX B TO 10 CFR PART 50
FEE CATEGORY: $10 B(2)$ $\cdots$ USE (S)

PROCUREMENT REPAIR USE MAINTENANCE

\footnotetext{
GENOA RADIATION SAFETY OFFICER 54632

GENOA
}

\begin{abstract}
COMMENTS :
\end{abstract}

\begin{abstract}
EXP IRATION DATE: $11 / 30 / 1999$
ORIG. ISSUE DATE: 09/17/1979

APPROVAL TYPE: SPECIAL FORM

REGULATION: SUBPART H TO 10 CFR PART 71
FEE CATEGORY: $10 B(2)$
\end{abstract}

USE (S)

PROCUREMENT
MAINTENANCE MAINTENAN USE

\section{COMMENTS:}

\author{
EXPIRATION DATE: 06/30/1999 \\ ORIG. I SSUE DATE: 03/29/1979 \\ APPROVAL TYPE: NORMAL AND SPECIAL FORM \\ REGULATION: APPENDIX B TO 10 CFR PART 50
FEE CATEGORY: 1 OB $(2)$
}

USE (S) MAINTENANCE REPAIR
PROCUREMENT 


\section{DOCKET\# : 07100026}

REVISION\# : 4

EXPIRATION DATE: $02 / 28 / 2000$
ORIG. ISSUE DATE: $12 / 04 / 1979$

APPROVAL TYPE: SPECIAL FORM EASTERN TESTING \& INSPECTION, INC.
P. O. BOX 447

THOROFARE

ATTN: MR. JOSEPH BADIALI

NJ 08086

REGULATION: SUBPART H TO 10 CFR PART 71
FEE CATEGORY: $10 B(2)$ USE(S)

PROCUREMENT MAINTENANCE REPAIR USE

COMMENTS:

DOCKET\# : 07100030

REVISION\#: 6

USER NAME: GENERAL ATOMICS

0. BOX 85608

3550 GENERAL ATOMICS COURT

SAN DIEGO

ATTN: MR. KEITH E. ASMUSSEN
CA 92186

\section{REVISION\# : 5}

GRINNELL CORPORATION
16O FRENCHTOWN ROAD

NORTH KINGSTOWN

ATTN: MR. WILLIAM GOLINI
RI 02852
EXPIRATION DATE: $06 / 30 / 2001$

APPROVAL TYPE: NORMAL AND SPECIAL FORM

REGULATION: APPENDIX B TO 10 CFR PART 50
FEE CATEGORY: $10 B(1)$
USE $(S)$

DESIGN

FABRICATION

ASSEMBLY

TESTING

MATUREMENT

MAINTE

MODIFICATION

USE
USER NAME: COMMENTS :
EXPIRATION DATE: 09/30/1999

APPROVAL TYPE: SPECIAL FORM

REGULATION: SUBPART H TO 10 CFR PART 71
USE (S)

PROCUREMENT

MAINTENANCE

REPAIR

USE 
REVISION\#: 8

USER NAME: AMERSHAM CORPORATION

40 NORTH AVENUE

BURLINGTON MA 01803

ATTN: MS. CATHLEEN ROUGHAN
EXPIRATION DATE: $10 / 31 / 1999$

APPROVAL TYPE: SPECIAL FORM

REGULATION: SUBPART H TO 10 CFR PART 71 FEE CATEGORY: $1 O B(1)$
$\operatorname{USE}(S)$

DESIGN

FABRICATION

ASSEMBLY

PROCUREMENT

MAINTENANCE

USE

COMMENTS:

DOCKET\# : 07100044

REVISION\#: 4

USER NAME: UNITED AIRLINES, SFOIQ - RSO

SAN FRANCISCO INTERNATIONAL AIRPORT SAN FRANCISCO

ATTN: MR LARRY MILLS

CA 94128

COMMENTS :

DOCKET\# : 07100045

REVISIONA: 5

USER NAME: NIAGARA MOHAWK POWER CORP .

\section{P. 0. BOX 32 LYCOMING \\ P. O. BOX
LYCOMING}

EXP IRATION DATE : 03/31/2000

APPROVAL TYPE: SPECIAL FORM

REGULATION: SUBPART H TO 10 CFR PART 71 FEE CATEGORY: $1 O B(2)$
$\operatorname{USE}(S)$

PROCUREMENT MAINTENANCE REPAIR USE
EXPIRATION DATE: 06/30/1999

APPROVAL TYPE: NORMAL AND SPECIAL FORM

REGULATION: APPENDIX B TO 10 CFR PART 50 FEE CATEGORY: $10 \mathrm{~B}(2)$

\section{USE(S)}

PROCUREMENT PROCUREMENT REPAIR USE

\section{COMMENTS :}




\section{DOCKETH : 07100051}

REVISIONA : 4

USER NAME: HARRISON STEEL CASTINGS CO.

ATTICA $\quad$ IN 47918

ATTN: MR. WILLIAM D. HOLLANDER

COMMENTS:

DOCKETH : 07100055

REVISION\# : 4

USER NAME: H. R. INSPECTION SERVICE

P.O. BOX 3280

SHAWNEE MISSION ${ }_{\text {ATTN: MR. ROBERT WILLIAMSON }}^{\text {KS } 66218}$

COMMENTS :
DOCKETH: 07100056

REVISION\# : 5

USER NAME: PECO ENERGY COMPANY

965 CHESTERBROOK BLVD.

WAYNE MR, G. A. HUNGER, JR, 190875691

COMMENTS:

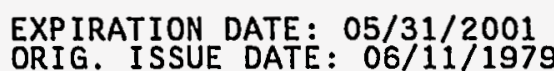

APPROVAL TYPE: SPECIAL FORM

FEE CATEGORY: $1 O B(2)$

EXPIRATION DATE: 09/30/1999

APPROVAL TYPE: SPECIAL FORM

REGULATION: SUBPART H TO 10 CFR PART 71

FEE CATEGORY: $1 O B(2)$
REGULATION: SUBPART H TO 10 CFR PART 71
USE $(S)$

USE

PROCUREMENT

REPAIR
MAINTENANCE

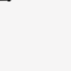

EXPIRATION DATE: $06 / 30 / 1999$

APPROVAL TYPE: NORMAL AND SPECIAL FORM

REGULATION: APPENDIX B TO 10 CFR PART 50

FEE CATEGORY: $10 B(2)$
USE(S)

MAINTENANCE

REPAIR

USE
PROCUREMENT

\section{COMMENTS:}


EXPIRATION DATE: $10 / 31 / 1999$

ORIG. ISSUE DATE: $01 / 15 / 1980$

USER NAME: INDUSTRIAL NUCLEAR COMPANY 2515 WILLIAMS STREE

SANTN: EANDRO W. HUDDLESTON

CA 94577
APPROVAL TYPE: NORMAL AND SPECIAL FORM

REGULATION: SUBPART H TO 10 CFR PART 71 FEE CATEGORY: $1 O B(1)$
USE(S)

ASSEMBLY ASSEMBLYTIOATION FABRICATION TESTING PROCUREMENT MAINTENANCE REPA

\section{COMMENTS :}

\section{DOCKET\# : 07100066}

REVISION\# : 4

USER NAME: CERTIFIED TESTING LABORATORIES, INC. 155 U.S. ROUTE 130

BORDENTOWN ATTN: MR. JOSEPH CUOZZO
EXPIRATION DATE: $09 / 30 / 1999$

ORIG. ISSUE DATE: 06/11/1979

APPROVAL TYPE: SPECIAL FORM

REGULATION: SUBPART H TO 10 CFR PART 71 FEE CATEGORY: $10 B(2)$

\begin{tabular}{l} 
USE(S) \\
\hline PROCUREMENT \\
MAINTENANCE \\
REPAIR \\
USE
\end{tabular}

COMMENTS:

DOCKETH : 07100068

\section{REVISION\#: 6} USER NAME: YANKEE ATOMIC ELECTRIC COMPANY
5BO MAIN STREET
BOLTON
ATTN: MR. JAY K. THAYER
MA 017401398

EXPIRATION DATE: $09 / 30 / 1999$

APPROVAL TYPE: NORMAL AND SPECIAL FORM REGULATION: APPENDIX B TO 10 CFR PART 50
FEE CATEGORY: $10 B(1)$
USE(S)

ASSEMBLY MODIFICATION FABRICATION TESTING DESIGN PROCUREMENT MAINTENANCE REPAIR USE

COMMENTS: 
DOCKETH : 07100069

REVISION\#: 5

USER NAME: CONSOLIDATED EDISON COMPANY BROADWAY \& BLEAKLEY AVE.

BUCHANAN

ATTN: MR. STEPHEN QUINN

NY 10511

COMMENTS:

DOCKETH : 07100075

REVISION\# : 4

USER NAME: NORFOLK SHIPBUILDING \& DRYDOCK COMPANY P.0. BOX 2100 NORFOLK ATTN: MR. CARL A. CHERRY

VA 235012100

COMMENTS :

DOCKETH : 07100079

REVISIONH : 4

USER NAME: BABCOCK \& WILCOX CO., NUCLEAR EQUIPMENT DIV. Si STIRLING AVENUE BÁRBERTON ATTN: MR. JAMES F. DRAIN
EXPIRATION DATE:
ORIG. I I SSUE DATE : $03 / 29 / 1999$
$03 / 1979$

APPROVAL TYPE: NORMAL AND SPECIAL FORM REGULATION: APPENDIX B TO 10 CFR PART 50
FEE CATEGORY: $108(2)$
USE (S)

PROCUREMENT MAINTENANCE REPAIR
EXPIRATION DATE: $09 / 30 / 1999$

APPROVAL TYPE: SPECIAL FORM

REGULATION: SUBPART H TO 10 CFR PART 71
FEE CATEGORY: $10 B(2)$
USE(S)

PROCUREMENT

MAINTENANCE

REPAIR
EXPIRATION DATE: $03 / 31 / 2000$
ORIG. ISSUE DATE: $11 / 26 / 1979$

APPROVAL TYPE: SPECIAL FORM

REGULATION: SUBPART H TO 10 CFR PART 71
FEE CATEGORY: $10 B(2)$
USE (S)

PROCUREMENT MAINTENANCE REPAIR USE

COMMENTS: 
EXPIRATION DATE : $03 / 31 / 2000$

ORIG. ISSUE DATE: 06/11/1979

USER NAME: TRANS WORLD AIRLINES

9200 N.W. 112 TH STREET ROOM 1 - $47 \dot{6}$

KANSAS CITY MO 641532003

ATTN: MR. ROBERT C. BAKER

COMMENTS :

DOCKETH : 07100083

\section{REVISION\# : 4}

USER NAME: NORTHERN STATES POWER CO

\section{WEST COUNTY ROAD 75
MONTICELLO \\ 2807 WEST COUNTY ROAD 75
MONTICELLO ATTN: MR. MARVIN ENGEN}

APPROVAL TYPE: SPECIAL FORM

REGULATION: SUBPART H TO 10 CFR PART 71 FEE CATEGORY: $10 B(2)$
USE (S)

USE MAINTENANCE PROCUREMENT REPAIR
EXPIRATION DATE : 06/30/1999 ORIG. ISSUE DATE: 04/02/1979

APPROVAL TYPE: NORMAL AND SPECIAL FORM

REGULATION: APPENDIX B TO 10 CFR PART 50 FEE CATEGORY: $10 B(1)$

\begin{tabular}{l} 
USE (S) \\
\hline DESIGN \\
FABRICATION \\
ASSEMBLY \\
TESTING \\
PROCUREMENT \\
MAINTENANCE \\
REPAIR \\
MODIFICATION \\
USE
\end{tabular}

COMMENTS :

DOCKET\# : 07100086

REVISION\# : 5

USER NAME: ALPHA-OMEGA SERVICES, INC. 9156 ROSE STREET, P. BOX 789

ATTN: MR. BRUCE HEDGER
CA 90706
EXPIRATION DATE : $08 / 31 / 1999$
ORIG. ISSUE DATE: $09 / 07 / 1979$

APPROVAL TYPE: SPECIAL FORM

REGULATION: SUBPART H TO 10 CFR PART 71
USE (S)

\section{DESIGN}

FABRICATION ASSEMBLY TESTING PROCUREMENT MAINTENANCE MOD IFICATION

COMMENTS : 
EXP IRATION DATE: $12 / 31 / 1999$

ORIG. ISSUE DATE: $12 / 10 / 1979$

APPROVAL TYPE: NORMAL AND SPECIAL FORM

REGULATION: SUBPART H TO 10 CFR PART 71
FEE CATEGORY: 1 OB(1)
USE (S)

DESIGN

ABRICATION

ASSEMBLY

TESTING

PROCUREMEN

MAINTENANCE

REPAIR

MODIFICATION

USE

COMMENTS :

DOCKETH : 07100090

REVISION\# : 4

USER NAME: COMBUSTION ENGINEER ING CO. . INC P. O. BOX 107

HEMATITE

ATTN: MR. IAN C. RICKARD

MO 63047

COMBUSTION ENGINEERING, INC. 2000 DAY HILL ROAD

WINDSOR

ATTN: MR. IAN C. RICKARD

CT 060950500
EXPIRATION DATE: $12 / 31 / 1998$
ORIG. ISSUE DATE: $05 / 02 / 1979$

APPROVAL TYPE: NORMAL AND SPECIAL FORM REGULATION: SUBPART H TO 10 CFR PART 71
FEE CATEGORY: $10 B(1)$

\section{$\operatorname{USE}(S)$}

\section{DESIGN}

FABRICATION

ASSEMBLY

TESTING

MAINTENANCE

REPAIR

MODIF ICATION

USE

COMMENTS :

DOCKETH : 07100093

REVISION\# : 4

USER NAME: HUNTINGTON TESTING INC.

HUNTINGTON

ATTN: MR. CLYDE W. MAY
WV 25717

\section{EXPIRATION DATE: $09 / 30 / 1999$}

APPROVAL TYPE: SPECIAL FORM

REGULATION: SUBPART H TO 10 CFR PART 71
USE ( $S$ )

PROCUREMENT MAINTENANCE REPAIR USE COMMENTS: 
DOCKET\# : 07100096

REVISION\#: 5

USER NAME: EG\&G FLORIDA, INC.

KENNEDY SPACE CENTER

ÁTTN: DR. PAUL V. HUMBERT

FL 32815

COMMENTS :

DOCKET\#: 07100097

REVISION\# : 5

USER NAME: NONDESTRUCTIVE INSPECTION SERVICE

P.0. BOX 220

HURRICANE WV 25526

ATTN: MR. H. M. HAULDREN

COMMENTS :

DOCKET\#： 07100098

REVISION\#: 4

USER NAME: NEWPORT NEWS SHIPBUILDING

4101 WASHINGTON AVENUE
EXPIRATION DATE: $06 / 30 / 2000$

ORIG. ISSUE DATE. 04/01/1980

APPROVAL TYPE: NORMAL AND SPECIAL FORM REGULATION: SUBPART H TO 10 CFR PART 71
FEE CATEGORY: 1 OB (2)
$\operatorname{USE}(S)$

PROCUREMENT MAINTENANCE USE

\section{NEWPORT NEWS
ATTN: MR. JOHN M. FLOWE VA 23607 \\ NEWPORT NEWS
ATTN: MR. JOHN M. FLOWE VA 23607}

COMMENTS :

EXPIRATION DATE: $06 / 30 / 2000$
ORIG. ISSUE DATE: $04 / 01 / 1980$

APPROVAL TYPE: SPECIAL FORM

REGULATION:
FEE CATEGORY: $10 B(2)$

\section{USE (S)}

PROCUREMENT MAINTENANCE REPAIR USE
EXPIRATION DATE: $01 / 31 / 2000$
ORIG. ISSUE DATE: $11 / 05 / 1979$

APPROVAL TYPE: SPECIAL FORM

REGULATION: SUBPART H TO 10 CFR PART 71
FEE CATEGORY: $10 B(2)$
USE(S)

PROCUREMENT MAINTENANCE REPAIR

USE 
EXPIRATION DATE: $09 / 30 / 1999$
ORIG. ISSUE DATE: $06 / 29 / 1979$

APPROVAL TYPE: SPECIAL FORM USER NAME: FOSTER WHEELER ENERGY CORPORATION
5900 STATE ROUTE 36

DANSVILLE NY 14437

COMMENTS :
REGULATION: SUBPART H TO 10 CFR PART 71
$\operatorname{USE}(S)$

PROCUREMENT

MAINTENANCE

REPAIR

USE

\section{DOCKETH: 07100100 \\ REVISION\#: 4 \\ USER NAME: VENEGAS IND. TEST LABS, INC. 13 PROGRESS AVE. \\ NASHUA ATTN: MR. MANUEL VENEGAS \\ NH 03060 \\ COMMENTS :}

DOCKET\# : 07100102

REVISION\# : 6

USER NAME: SOURCE PRODUCTION AND EQUIPMENT CO., INC. 113 TEAL STREET

ST. ROSE

R. DICHARRY
LA 700879691
EXPIRATION DATE: $09 / 30 / 1999$
ORIG. ISSUE DATE: $06 / 11 / 1979$

APPROVAL TYPE: SPECIAL FORM

REGULATION: SUBPART H TO 10 CFR PART 71
USE (S)

PROCUREMENT MAINTENANCE REPAIR

USE
EXPIRATION DATE: $07 / 31 / 2001$
ORIG. ISSUE DATE: $12 / 18 / 1980$

APPROVAL TYPE: NORMAL AND SPECIAL FORM

REGULATION: SUBPART H TO 10 CFR PART 71 FEE CATEGORY: 1OB(1)

COMMENTS: 
NRC-1265006-006

U.S. NUCLEAR REGULATORY COMMISSION

QUAL ITY ASSURANCE PROGRAMS REPORT

$10 / 01 / 1996$

DOCKETH : 07100103

REVISION\# : 6

USER NAME: MAXIM TECHNOLOGIES, INC.

662 CROMWELL AVE.

ST. PAUL

COMMENTS :

DOCKETH : 07100108

REVISION\# : 4

USER NAME: UNIVERSITY OF MISSOURI-COLUMBIA ROOM 402 RESEARCH REACTOR FACILITY

ATTN: MR. WALT A. MEYER, JR.

MO 65211

COMMENTS :

DOCKETH : 07100110

REVISIONH : 4

USER NAME: BUCKEYE STEEL CASTINGS COMPANY 2211 PARSONS AVENUE

COLUMBUS

ATTN: MR. DELNO SEITZ
EXPIRATION DATE: 04/30/1999

APPROVAL TYPE: SPECIAL FORM

REGULATION: SUBPART H TO 10 CFR PART 71
FEE CATEGORY: $10 B(2)$
USE (S)

PROCUREMENT

MAINTENANCE

REPAIR

USE
EXPIRATION DATE: $03 / 31 / 1999$
ORIG. ISSUE DATE:

APPROVAL TYPE: SPECIAL FORM

REGULATION: SUBPART H TO 10 CFR PART 71 FEE CATEGORY: EDUC
USE(S)

PROCUREMENT MAINTENANCE REPAIR

USE

\section{COMMENTS:}

EXPIRATION DATE: $09 / 30 / 1999$
ORIG. I SSUE DATE: $04 / 27 / 1979$

APPROVAL TYPE: SPECIAL FORM

REGULATION: SUBPART H TO 10 CFR PART 71 REGULATION: SUBPART
FEE CATEGORY: $1 O B(2)$

\section{USE (S)}

PROCUREMENT MAINTENANCE REPAIR 
EXPIRATION DATE: $10 / 31 / 1999$

ORIG. ISSUE DATE: 08/03/1979

APPROVAL TYPE: SPECIAL FORM

MATERIAL TESTING LABORATORIES, INC.

1531 EARLY STREET

NORFOLK ATTN: MR. JAMES R. CANNON VA 235021603

FEE CATEGORY: $1 O B(2)$

REGULATION: SUBPART H TO 10 CFR PART 71

\begin{tabular}{l} 
USE $(S)$ \\
\hline PROCUREMENT \\
MAINTENANCE \\
REPAIR \\
USE
\end{tabular}

\section{COMMENTS:}

DOCKET\#: 07100113

REVISION\# : 4

EXPIRATION DATE : $10 / 31 / 1999$

ORIG. ISSUE DATE: 06/11/1979

APPROVAL TYPE: SPECIAL FORM

NOOTER CORPORATION
1400 SOUTH THIRD STREET

ATTIN: MR. DENNIS L. FRAZIER MO 63104

REGULATION: SUBPART H TO 10 CFR PART 71

FEE CATEGORY: $1 O B(2)$

\section{USE (S) \\ PROCUREMENT MAINTENANCE REPAIR}

\section{COMMENTS:}

DOCKETH : 07100114

REVISIONA :

USER NAME: COLBY-THIELMEIER TESTING CO IN 10627 LIBERTY STREET

ATTIN! MOUIS. L.F. THIELMEIER MO 63132

COMMENTS:

EXPIRATION DATE: $03 / 31 / 2000$

ORIG. I SSUE DATE: $12 / 17 / 1979$

APPROVAL TYPE: SPECIAL FORM

REGULATION: SUBPART H TO 10 CFR PART 71
USE(S)

PROCUREMENT

MAINTENANCE

REPAIR
USE 


\section{DOCKET\# : 07100115}

REVISION\#: 8

EXP IRATION DATE: 08/31/2001 USER NAME: FRAMATOME COGEMA FUELS
P.O. BOX 11646

LYNCHBURG VA

APPROVAL TYPE: NORMAL AND SPECIAL FORM

REGULATION: SUBPART H TO 10 CFR PART 71 ATTN: MS. GAYLE F. ELLIOTT FEE CATEGORY: 1OB(1)

COMMENTS :

DOCKET\# : 07100119

REVISION\#: 4

USER NAME: INGERSOLL-RAND CO

942 MEMORIAL PARKWIAY

PHILLIPSBURG
ATTN: MR. MATTHEW M. MORGANELL 08865

ATTN: MR. MATTHEW M. MORGANELL

COMMENTS :

DOCKET\# : 07100121

REVISION\#: 6

USER NAME: NEUTRON PRODUCTS, INC. 22301 MT. EPHRAIM ROAD

DICKERSON

ATTN: MR. FRANK SCHWOERER
MD 20842
EXPIRATION DATE : 09/30/1999

ORIG. ISSUE DATE: 06/11/1979

APPROVAL TYPE: SPECIAL FORM

REGULATION: SUBPART H TO 10 -CFR PART 71 FEE CATEGORY: $10 B(2)$
$\operatorname{USE}(S)$

DESIGN

FABRICATION

ASSEMBLY

TESTING

PROCUREMENT

MAINTENANCE

REPAIR

MODIFICATION

USE
EXP IRATION DATE: $01 / 31 / 2001$
ORIG.- ISSUE DATE: $06 / 06 / 1979$

APPROVAL TYPE: NORMAL AND SPECIAL FORM

REGULATION: SUBPART H TO 10 CFR PART 71
FEE CATEGORY: $10 B(1)$
$\operatorname{USE}(S)$

ASSEMBLY

FABRICATION

TESTING

PESIGN

MAINTENANCE

REPAIR

COMMENTS 
DOCKET\# : 07100122

REVISION\#: 5

USER NAME: J. L. SHEPHERD \& ASSOCIATES 1010 ARROYO ST.

SAN FERNANDO

ATTN: MR. J. L. SHEPHERD

CA 91340
EXPIRATION DATE: $01 / 31 / 2001$

APPROVAL TYPE: NORMAL AND SPECIAL FORM

REGULATION: SUBPART H TO 10 CFR PART 71 FEE CATEGORY: 1OB(1)

COMMENTS :

DOCKETH : 07100131

\section{REVISION\#: 4}

USER NAME: TESTING INSTITUTE OF ALASKA, INC. TEST RAILROAD AVE.

ANCHORAGE
ATTN: MR. DONALD M. LOCKMAN AK 99501
EXPIRATION DATE: $04 / 30 / 2000$ ORIG. ISSUE DATE : 05/21/1979

APPROVAL TYPE: SPECIAL FORM

REGULATION: SUBPART H TO 10 CFR PART 71
FEE CATEGORY: $10 B(2)$
USE(S)

DESIGN

FABRICATION

ASSEMBLY

TESTING

PROCUREMENT

MAINTENANCE

REPAIR

MODIFICATION

USE

\section{COMMENTS :}

DOCKET\# : 07100135

REVISION\#: 6

USER NAME: B\&W NUCLEAR ENVIRONMENTAL SERVICES, INC. R.D. 1, BOX 355 VANDERGRIFT ATTN: MR. D. K. SGARLATA

PA 15690
EXPIRATION DATE: $12 / 31 / 2000$

APPROVAL TYPE: SPECIAL FORM

REGULATION: SUBPART H TO 10 CFR PART 71
USE(S)

PROCUREMENT MAINTENANCE REPAIR

\section{COMMENTS:}




\section{DOCKETH: 07100138}

REVISION\# : 4

USER NAME: BATTELLE COLUMBUS LABORATORY 505 KING AVENUE

COLUMBUS DTTN: MR. DENNIS CLUM
$\mathrm{OH} 43201$
EXPIRATI ION DATE: $08 / 31 / 1996$
ORIG. ISSUE DATE : $02 / 12 / 1981$

APPROVAL TYPE: NORMAL AND SPECIAL FORM

REGULATION: SUBPART H TO 10 CFR PART 71 FEE CATEGORY: 1OB(1)
USE (S)

USE

MAINTENANCE

ASSEMBLY

MODIFICATION

PROCUREMENT

FABRICATION

REPAIR

TESTING

\section{COMMENTS :}

DOCKETH : 07100139

REVISION\# : 4

USER NAME: TULSA GAMMA RAY, INC.

1127 SOUTH LEWI

TULSA MR. JAMES MOSS

OK 74104

COMMENTS:

DOCKETH : 07100146

REVISIONH : 4

USER NAME: GLOBE X-RAY SERVICES, INC 8441 SOUTH UNION

TULSA MR. KENNETH SWANSON
OK 74132
EXP IRATION DATE: $12 / 31 / 2000$

ORIG. I SSUE DATE: $07 / 15 / 1980$

APPROVAL TYPE: SPECIAL FORM

REGULATION: SUBPART H TO 10 CFR PART 71 FEE CATEGORY: $10 B(2)$
USE (S)

PROCUREMENT MAINTENANCE REPAIR

USE

\section{COMMENTS:}

EXPIRATION DATE: $10 / 31 / 1999$
ORIG. ISSUE DATE : $07 / 19 / 1979$

APPROVAL TYPE: SPECIAL FORM

REGULATION: SUBPART H TO 10 CFR PART 71
FEE CATEGORY: $10 B(2)$

\begin{tabular}{l} 
USE(S) \\
\hline PROCUREMENT \\
MAINTENANCE \\
REPAIR \\
USE
\end{tabular}

MAINTENANCE

USE 


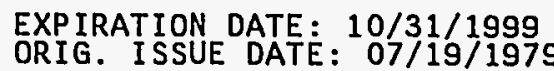

APPROVAL TYPE: SPECIAL FORM

USER NAME: BATH IRON WORKS CORP.
700 WASHINGTON STREET

BATH ME 04530
ATTN: MR. JOHN H. BERGER

COMMENTS:
REVISIONA : 4

USER NAME: FROEHLING \& ROBERTSON, INC 3015 DUMBARTON ROAD, BOX 27524

RICHMOND ATTN: MR. WILLIAM BRIODY

VA 232617524

REGULATION: SUBPART H TO 10 CFR PART 71 USE(S)

PROCUREMENT MAINTENANCE REPAIR USE

\section{COMMENTS :}

DOCKETH : 07100158

REVISION\#: 4

USER NAME: X-RAY INDUSTRIES, INC.

1961 THUNDERBIRD' STREET

TROY MTTN: MR. LAWRENCE H. REDHAGE 48084
EXPIRATION DATE: $08 / 31 / 1999$
ORIG. I SSUE DATE: $06 / 28 / 1979$

APPROVAL TYPE: SPECIAL FORM

REGULATION: SUBPART H TO 10 CFR PART 71
USE (S)

PROCUREMENT MAINTENANCE REPAIR

COMMENTS:

EXPIRATION DATE: $01 / 31 / 2000$
ORIG. ISSUE DATE: $07 / 19 / 1979$

APPROVAL TYPE: SPECIAL FORM

REGULATION: SUBPART H TO 10 CFR PART 71
USE(S)

USE

MAINTENANCE PROCUREMENT REPAIR 


\section{DOCKETH : 07100162}

\section{REVISION\#: 5}

USER NAME: RAYTHEON ENGINEERS AND CONSTRUCTORS, INC., EBASCO 210 CLAY AVENUE

LYNDHURST

ATTN: MR. HAROLD BULLEN

COMMENTS :

\section{REVISION\#: 6}

USER NAME: MASSACHUSETTS INSTITUTE OF TECHNOLOGY 138 ALBANY STREET

CAMBRIDGE
ATTN: MR. THOMAS H. NEWTON, MA 02139

COMMENTS:

DOCKETH : 07100168

\section{REVISION\# : 5}

USER NAME: QUALITY ASSURANCE LABS

BO PLEASANT AVENUE

\section{PLEASANT AV
SOUTH PORTLAND \\ ATTN: MR. ROBERT PARECHANIANE 04106}

EXPIRATION DATE: $12 / 31 / 1999$

APPROVAL TYPE: SPECIAL FORM

REGULATION: SUBPART H TO 10 CFR PART 71

FEE CATEGORY: $10 B(2)$
USE (S)

PROCUREMEN MAINTENANCE

REPAIR

USE
EXPIRATION DATE: 06/30/2001

APPROVAL TYPE: SPECIAL FORM

REGULATION: SUBPART H TO 10 CFR PART 71

FEE CATEGORY: EDUC

\section{USE(S)}

PROCUREMENT

MAINTENANCE

REPAIR

USE

\section{COMMENTS :}

EXPIRATION DATE: $11 / 30 / 1999$
ORIG. I SSUE DATE: $06 / 29 / 1979$

APPROVAL TYPE: SPECIAL FORM

REGULATION: SUBPART H TO 10 CFR PART 71

FEE CATEGORY: $10 B(2)$

\begin{tabular}{l} 
USE (S) \\
\hline USE \\
MAINTENANCE \\
PROCUREMENT \\
REPAIR
\end{tabular}

MAINTENANCE REPAIR 


\section{DOCKET\#：07100169}

REVISION\#: 5

USER NAME: FLORIDA POWER \& LIGHT COMPANY

P. 0. BOX 14000

JUNO BEACH

ATTN: MR. ROBERT ACOSTA
FL 334080420
EXPIRATION DATE: $08 / 31 / 1999$
ORIG. ISSUE DATE: $05 / 04 / 1979$

APPROVAL TYPE: NORMAL AND SPECIAL FORM REGULATION: APPENDIX B TO 10 CFR PART 50
FEE CATEGORY: $10 B(2)$
$\operatorname{USE}(S)$

PROCUREMENT MAINTENANCE REPAIR

COMMENTS:

DOCKET\# : 07100170

\section{REVISION\#: 6}

USER NAME: GENERAL ELECTRIC COMPANY P. 0 BOX 460 , VALLECITOS ROAD VALLECITOS NUCLEAR CENTER PLEASANTON ATTN: MR. G. E. CUNNINGHAM
CA 94566
EXPIRATION DATE : $11 / 30 / 2000$

ORIG. ISSUE DATE: $04 / 30 / 1980$

APPROVAL TYPE: NORMAL AND SPECIAL FORM

REGULATION: SUBPART H TO 10 CFR PART 71 FEE CATEGORY: IOB(1)

\begin{tabular}{l} 
USE(S) \\
\hline DESIGN \\
FABRICATION \\
ASSEMBLY \\
TESTING \\
PROCUREMENT \\
MAINTENANCE \\
REPAIR \\
MODIFICATION \\
USE
\end{tabular}

COMMENTS :

DOCKET\# : 07100171

REVISION\# : 5

USER NAME: GLITSCH FIELD SERVICES/NDE, INC. 5250 MAYFAIR ROAD. P. O. BOX 2908

NORTH CANTON
EXPIRATION DATE: $11 / 30 / 1999$

APPROVAL TYPE: SPECIAL FORM

REGULATION: SUBPART H TO 10 CFR PART 71
FEE CATEGORY: $10 B(2)$
USE( $(S)$

\section{USE}

MAINTENANCE PROCUREMENT REPAIR

COMMENTS : 
DOCKETH : 07100174

REVISION\#: 5

USER NAME: SOUTHERN CALIFORNIA EDISON COMPANY 23 PARKER STREET

IRVINE ATTN: MR, WALTER C. MARSH CA 92718

COMMENTS :

DOCKETH : 07100179

REVISION\# : 5

USER NAME: NUCLEAR CONTAINERS, INC. 125 IODENT WAY, SUITE B

\section{ELIZABETHTON
ATTN: MR. WILLIAM M. ARNOLD TN 37643}

ORIG. I SSUE DATE: $05 / 08 / 1979$

APPROVAL TYPE: NORMAL AND SPECIAL FORM

REGULATION: APPENDIX B TO 10 CFR PART 50 FEE CATEGORY: 1OB(2)
USE (S)

PROCUREMENT

MAINTENANCE

REPAIR

USE

ATTN: MR. WILLIAM M. ARNOLO

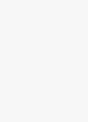

COMMENTS:

EXPIRATION DATE: $05 / 31 / 2001$

APPROVAL TYPE: NORMAL AND SPECIAL FORM

REGULATION: SUBPART H TO 10 CFR PART 71 FEE CATEGORY: 1OB(1)

\begin{tabular}{l} 
USE(S) \\
\hline DESIGN \\
FABRICATION \\
ASSEMBLY \\
TESTING \\
PROCUREMENT \\
MAINTENANCE \\
REPAIR \\
MODIFICATION \\
USE
\end{tabular}

DOCKETH : 07100181

REVISIONH : 4

EXPIRATION DATE: $10 / 31 / 1999$
ORIG. ISSUE DATE : $06 / 29 / 1979$

USE(S)

USER NAME: CENTURY INSPECTION, INC.

P.0. BOX 59126

APPROVAL TYPE: SPECIAL FORM

DALLAS ATTN: MR. DREW H. MCCORKLE TX 752291126

REGULATION: SUBPART H TO 10 CFR PART 71
FEE CATEGORY: $10 B(2)$

PROCUREMENT

MAINTENANCE

REPAIR

USE

COMMENTS: 
DOCKETH : 07100185

REVISION\#: 4

USER NAME: FBF, INC.

1201 HILTON RD.

KNOXVILLE
ATTN: MR. H. STRINGFIELD TN 37921
USE (S)

EXPIRATION DATE: $08 / 31 / 2000$

APPROVAL TYPE: NORMAL AND SPECIAL FORM

REGULATION: SUBPART H TO 10 CFR PART 71 FEE CATEGORY: 1OB (1)
DESIGN

FABRICATION

ASSEMBLY

ASSEMBLY

PROCUREMENT

MAINTENANCE

REPAIR

MOPATCATION

USE

\section{COMMENTS:}

DOCKETH : 07100186

REVISION\# : 4 USER NAME: HIGH STEEL STRUCTURES, INC.

LANCASTER ATTN: MR. JERE LONG

PA 17605

COMMENTS :

DOCKET\# : 07100192

REVISION\# : 6

USER NAME: VECTRA FUEL SERVICES 6203 SAN IGNACIO AVENUE SUITE 100 ATTN: MR. JACK ROLLINS

CA 95119

VECTRA TECHNOLOGIES, INC. BROOKSIDE OFFICE PARK NO. 1, HARBISON WAY, \#209 COLUMBIA ANTHONY L PATKO SC 29212
EXPIRATION DATE: $10 / 31 / 1999$ ORIG. ISSUE DATE: 07/19/1979

APPROVAL TYPE: SPECIAL FORM

REGULATION: SUBPART H TO 10 CFR PART 71
FEE CATEGORY: $10 B(2)$

\begin{tabular}{l} 
USE(S) \\
\hline PROCUREMENT \\
MAINTENANCE \\
REPAIR \\
USE
\end{tabular}

N NTENANCE REPAIR USE

COMMENTS:

EXPIRATION DATE: $09 / 30 / 2000$ ORIG. I SSUE DATE: 09/10/1980

APPROVAL TYPE: NORMAL AND SPECIAL FORM REGULATION: SUBPART H TO 10 CFR PART 71
FEE CATEGORY: $10 B(1)$
USE(S)

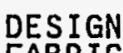

FABRICATION ASSEMBLY TESTING PROCUREMENT MAINTENANCE REPAIR MODIFICATION 


\section{DOCKET\# : 07100194}

REVISION\#: 6

USER NAME: VIRGINIA ELECTRIC AND POWER COMPANY P. O. BOX 26666

RICHMOND M. STEWART VA 23261

COMMENTS :
DOCKET\# : 07100195

\section{REVISION\#: 5}

USER NAME: TOLEDO EDISON COMPANY, DAVIS-BESSE UNIT NO. 1 P. O. BOX 94661 ALEVELAND MR. DONALD C. SHELTON ${ }^{\text {OH } 441014661}$
EXPIRATION DATE: 05/31/1999

APPROVAL TYPE: SPECIAL FORM FEE CATEGORY: $10 B(2)$
REGULATION: APPENDIX B TO 10 CFR PART 50
USE (S)

PROCUREMENT

MAINTENANCE

REPAIR

USE
COMMENTS :
DOCKET\# : 07100201

REVISION\# : 4

USER NAME: DURALDY TECHNOLOGIES, INC
EXPIRATION DATE : $08 / 31 / 1999$

ORIG. ISSUE DATE: 04/30/1979

APPROVAL TYPE: NORMAL AND SPECIAL FORM

REGULATION: APPENDIX B TO 10 CFR PART 50

FEE CATEGORY: $10 B(2)$
USE (S)

PROCUREMENT MAINTENANCE REPAIR USE

\section{BRIDGE STREET, P.O. BOXX 81
SCOTTDALE \\ 120 BRIDGE STREET, P.O. BOX 81
SCOTTDALE
ATTN: MR. MARK DOVE, III PA 15683 \\ 120 BRIDGE STREET, P.O. BOX 81
SCOTTDALE}

COMMENTS:

\section{EXPIRATION DATE: $12 / 31 / 1999$ \\ ORIG. ISSUE DATE : 08/09/1979 \\ APPROVAL TYPE: SPECIAL FORM \\ REGULATION: SUBPART H TO 10 CFR PART 71 \\ FEE CATEGORY: $10 B(2)$}

\section{USE (S)}

PROCUREMENT REPAIR USE MAINTENANCE 
PACIFIC GAS \& ELECTRIC CO
77 BEALE STREET, ROOM $145 \mathrm{i}$

SAN FRANCISCO

ATTN: MR. GREGORY M. RUEGER ${ }^{\text {CA } 94177}$
EXP IRATION DATE: 06/30/1999

ORIG. ISSUE DATE: 05/02/1979

APPROVAL TYPE: NORMAL AND SPECIAL FORM

REGULATION: APPENDIX B TO 10 CFR PART 50
FEE CATEGORY: $10 B(2)$

USE (S)

PROCUREMENT MAINTENANCE REPAIR

COMMENTS:

DOCKETH: 07100206

REVISION\# : 5 USER NAME: GREDE-PRYOR, INC., C/O GREDE FOUNDRIES, INC.
P.0. BOX 26499

MILWAUKEE ATTN: MR. JAMES 0 . WHITE

WI 532260499

COMMENTS :

DOCKET\# : 07100207

REVISION\# : 8 USER NAME: CONNECTICUT YANKEE ATOMIC POWER COMPANY

EAST HAMPTON

NORTHEAST NUCLEAR ENERGY COMPANY
P. O. BOX 270

HARTFORD

ATTN: MR. J. F. OPEKA

CT 061410270
EXPIRATION DATE: $03 / 31 / 1999$
ORIG. ISSUE DATE: $12 / 17 / 1979$

APPROVAL TYPE: SPECIAL FORM

REGULATION: SUBPART H TO 10 CFR PART 71
USE(S)

PROCUREMENT MAINTENANCE REPAIR 


\section{DOCKETH : 07100211}

REVISION\# : 5

USER NAME: BOSTON EDISON COMPANY PILGRIM NUCLEAR POWER STA. ROCKY HILL ROAD PLYMOUTH MA 023605599 ATTN: REGULATORY AFFAIRS MGR.
EXPIRATION DATE: $11 / 30 / 1999$

APPROVAL TYPE: NORMAL AND SPECIAL FORM

REGULATION: APPENDIX B TO 10 CFR PART 50

FEE CATEGORY: $1 O B(2)$

PROCUREMENT MAINTENANCE REPAIR USE

\section{COMMENTS :}

DOCKET\#： 07100213

REVISION\# : 4

USER NAME: INTERMOUNTAIN TESTING CO 2965 SOUTH SHOSHONE STREE ENGLEWOOD ATTN: MR. GARY BOLLERUD
CO 80110
EXP IRATION DATE: $01 / 31 / 2000$

ORIG. ISSUE DATE: 06/29/1979

APPROVAL TYPE: SPECIAL FORM

REGULATION: SUBPART H TO 10 CFR PART 71 FEE CATEGORY: $10 B(2)$

COMMENTS :

DOCKET\#： 07100215

REVISION\# : 6

USER NAME: SPACE SCIENCE SERVICES, INC. ROUTE. 6, BOX 210

NAPIER FIELD

ATTN: MS. NINA WALKER
EXPIRATION DATE: $03 / 31 / 2000$

APPROVAL TYPE: SPECIAL FORM

REGULATION: SUBPART H TO 10 CFR PART 71 FEE CATEGORY: 1OB(2)

\section{USE(S)}

PROCUREMENT MAINTENANCE MEPAIR USE

\section{COMMENTS :}




\section{DOCKET\#：07100216 REVISION\#: 6}

USER NAME: CONAM INSPECTION INC.
1245 W. NORWOOD AVENUE

ATASCA ATR. ROBERT J. SLACK IL 60143

COMMENTS :

DOCKET\# : 07100220

REVISIONA: 5

USER NAME: ELECTRIC BOAT CORPORATION 75 EASTERN POINT ROAD

GROTN: MR. GARRY BALESTRACCI ${ }^{\text {CT } 063404989}$ \\ GROTON \\ GARY CT 063404989}

EXP IRATION DATE: 09/30/1999

APPROVAL TYPE: SPECIAL FORM

REGULATION: SUBPART H TO 10 CFR PART 71 FEE CATEGORY: $10 B(2)$
USE (S)

PROCUREMENT PROCUREMENT MAINTENANC USE

COMMENTS:

DOCKET\# : 07100221

REVISIONH : 4

USER NAME: PRECISION COMPONENTS CORP. P. O. BOX 15101

YORK ATTN: MR. FRANCIS G. KARCHNAK 17405

COMMENTS:

EXPIRATION DATE: $02 / 28 / 2001$
ORIG. I SSUE DATE: $06 / 29 / 1979$

APPROVAL TYPE: SPECIAL FORM

REGULATION: SUBPART H TO 10 CFR PART 71

FEE CATEGORY: $10 B(2)$

\section{USE (S)}

PROCUREMENT MAINTENANCE REPAIR USE

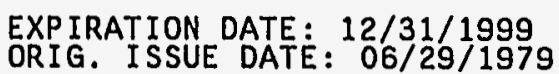

APPROVAL TYPE: SPECIAL FORM

REGULATION: SUBPART H TO 10 CFR PART 71
USE (S)

PROCUREMENT MAINTENANCE REPAIR 
EXPIRATION DATE: $08 / 31 / 1999$
ORIG. ISSUE DATE : $06 / 29 / 1979$

APPROVAL TYPE: SPECIAL FORM

ST. LOUIS

ATTN: MR. ROBIN SINN
MO 63103

USER NAME: ST, LOUIS TESTING LABORATORIES, INC.
2810 CLARK AVE.

COMMENTS : REGULATION: SUBPART H TO 10 CFR PART 71
FEE CATEGORY: $10 B(2)$

$\operatorname{USE}(S)$

\section{PROCUREMENT} MAINTENANCE REPAIR

\section{DOCKET\# : 07100226 \\ REVISION\# : 5}

USER NAME: STATE UNIVERSITY OF NEW YORK AT BUFFALO ROTARY ROAD 3435 MAIN STREET BUFFALO NY 14214

ATTN: MR, DANIEL W. SULLIVAN
EXPIRATION DATE: $06 / 30 / 2000$

ORIG. ISSUE DATE:

APPROVAL TYPE: SPECIAL FORM

REGULATION: SUBPART H TO 10 CFR PART 71

FEE CATEGORY: EDUC

\section{$\operatorname{USE}(S)$}

PROCUREMENT MAINTENANCE REPAIR USE

COMMENTS :

DOCKET\#: 07100227

REVISION\#: 10

USER NAME: TENNESSEE VALLEY AUTHORITY

1101 MARKET STREET

CHATTANOOGA

ATTN: MR. RAUL R. BARON
TN 374022801
EXP IRAT ION DATE : 08/31/1999

APPROVAL TYPE: NORMAL AND SPECIAL FORM

REGULATION: APPENDIX B TO 10 CFR PART 50 FEE CATEGORY: $10 B(1)$

\begin{tabular}{l} 
USE (S) \\
\hline ASSEMBLY \\
MODIFICATION \\
FABRICATION \\
TESTING \\
DESIGN \\
PROCUREMENT \\
MAINTENANCE \\
REPAIR \\
USE
\end{tabular}

COMMENTS : 
EXPIRATION DATE : $11 / 30 / 1999$

ORIG. ISSUE DATE: 09/10/1979

APPROVAL TYPE: SPECIAL FORM

CAPITAL X-RAY
P.0. BOX 3264

ATTSA MR. GEORGE W. JOHNSON OK 74101

COMMENTS :

\section{REVISION\#: 6}

USER NAME : CHEM-NUCLEAR SYSTEMS, INC. 140 STONERIDGE DRIVE

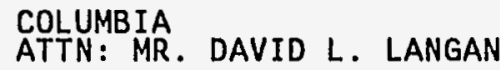

SC 29210
REGULATION: SUBPART H TO 10 CFR PART 71
$\operatorname{USE}(S)$

PROCUREMENT MAINTENANC REPAIR

USE
EXPIRATION DATE: $08 / 31 / 1999$
ORIG. ISSUE DATE: $10 / 25 / 1979$

APPROVAL TYPE: NORMAL AND SPECIAL FORM REGULATION: SUBPART H TO 10 CFR PART 71
FEE CATEGORY: $1 O B(1)$

COMMENTS :

DOCKETH : 07100234

REVISION\# : 4

USER NAME: X-RAY INC.

7500 PERIMETER RD. SOUTH

SEATTLE
EXPIRATION DATE: $11 / 30 / 1999$
ORIG. ISSUE DATE: $06 / 29 / 1979$

APPROVAL TYPE: SPECIAL FORM

REGULATION: SUBPART H TO 10 CFR PART 71
FEE CATEGORY: $10 B(2)$
USE(S)

\section{DESIGN}

FABRICATION

ASSEMBLY

TESTING

MAINTENANCE REPAIR

MODIFICATION

USE

COMMENTS : 


\section{DOCKET\# : 07100236}

REVISION\#: 4

USER NAME: AMERICAN AIRLINES, MAINTENANCE \& ENGR. CENTER P.0.BOX 582809

TULSA MR. BURL W. NETHERCUTT 741582809

COMMENTS:
REVISION\# : 4

USER NAME: DEPARTMENT OF AGRICULTURE 6303 IVY LANE

GREENBELT JOHN T. JENSEN

MD 207701433
EXPIRATION DATE: $01 / 31 / 2000$

ORIG. ISSUE DATE: 09/07/1979

APPROVAL TYPE: SPECIAL FORM

REGULATION: SUBPART H TO 10 CFR PART 71 FEE CATEGORY: $1 O B(2)$
USE ( $S$ )

PROCUREMENT MAINTENANCE MAPATEN USE
EXPIRATION DATE: $02 / 28 / 2000$

APPROVAL TYPE: NORMAL AND SPECIAL FORM

REGULATION: SUBPART H TO 10 CFR PART 71 FEE CATEGORY: IOB(1)

\begin{tabular}{l} 
USE(S) \\
\hline ASSEMBLY \\
MODIFICATION \\
FABRICATION \\
TESTING \\
DESIGN \\
PROCUREMENT \\
MAINTENANCE \\
REPAIR \\
USE
\end{tabular}

COMMENTS :

DOCKETH: 07100240

REVISION\# : 6

USER NAME: NEW YORK POWER AUTHORITY

123 MAIN STREET

WHITE PLAINS

ATTN: MR. W. A. JOSIGER

NY 10601

NEW YORK POWER AUTHORITY, INDIAN POINT UNIT. \# 3

BUCHANAN

ATTN: MR. PAUL SAUNDERS

NY 10511
EXPIRATION DATE: $10 / 31 / 1999$

APPROVAL TYPE: NORMAL AND SPECIAL FORM

REGULATION: APPENDIX B TO 10 CFR PART 50 FEE CATEGORY: $1 O B(2)$

\section{USE(S)}

PROCUREMENT MAINTENANCE REPAIR

USE

COMMENTS : 
REVISION\# : 4

USER NAME: LAW ENGINEERING INC.

P.O. BOX 888013

3 RAVINIA DRIVE, SUITE 1830

ATLANTA A DAVID A. RUMRILL GA 30346

COMMENTS :

DOCKET\# : 07100249

REVISIONH : 5

USER NAME: NUCLEAR FUEL SERVICES, INC.
$P .0$. BOX 337, MS 123
ERWIN ATTN: MR. ANDREW M. MAXIN
TN 37650

EXPIRATION DATE: $01 / 31 / 2000$

ORIG. ISSUE DATE: 06/29/1979

APPROVAL TYPE: SPECIAL FORM

REGULATION: SUBPART H TO 10 CFR PART 71
FEE CATEGORY: $10 B(2)$
EXPIRATION DATE: $01 / 31 / 2001$
ORIG. ISSUE DATE: $07 / 10 / 1980$

APPROVAL TYPE: NORMAL AND SPECIAL FORM

REGULATION: SUBPART H TO 10 CFR PART 71

\begin{tabular}{l} 
USE(S) \\
\hline PROCUREMENT \\
MAINTENANCE \\
REPAIR \\
USE
\end{tabular}

DOCURENT

\section{COMMENTS :}

DOCKET\# : 07100250

REVISIONH : 4

USER NAME: TRANSNUCLEAR, INC.

HAWTHORNE

ATTN: MR. JOHN MANGUSI
NY 105322120
EXPIRATION DATE: $06 / 30 / 2000$
ORIG. ISSUE DATE: $03 / 05 / 1980$

APPROVAL TYPE: NORMAL AND SPECIAL FORM

REGULATION: SUBPART H TO 10 CFR PART 71
USE (S)

ASSEMBLY

MOD IFICATION

FABRICATION

TESTING

DESIGN

USE

REPAIR

PROCUREMENT

MAINTENANCE 
DOCKET\# : 07100252

\section{REVISION\#: 3}

USER NAME: DAYTON X-RAY COMPANY

705 ALBANY STREET

DAYTON RICHARD J SAMMONS 45408

COMMENTS :
APPROVAL TYPE: SPECIAL FORM

REGULATION: SUBPART H TO 10 CFR PART 71

\section{EXPIRATION DATE: 03/31/1997}

$\operatorname{USE}(S)$

PROCUREMENT MAINTENANCE REPAIR USE

\section{DOCKETH : 07100253}

REVISION\# : 4

USER NAME: TWIN PORTS TESTING, INC. 1301 NORTH THIRD STREET SUPERIOR
ATTN: MR. JERRY RUSSELL

COMMENTS :
REVISION\# : 4

USER NAME: GENERAL ELECTRIC COMPANY, NUCLEAR ENERGY P. 0. BOX 780

WILMINGTON

ATTN: DR. RALPH REDA
NC 28402
EXPIRATION DATE: $11 / 30 / 1999$
ORIG. ISSUE DATE: $06 / 29 / 1979$

APPROVAL TYPE: SPECIAL FORM

REGULATION: SUBPART H TO 10 CFR PART 71 FEE CATEGORY: 1 OB (2)

\begin{tabular}{l} 
USE (S) \\
\hline PROCUREMENT \\
MAINTENANCE \\
REPAIR \\
USE
\end{tabular}

\section{DOCKET\# : 07100254}

NC 28402

FEE CATEGORY: $10 B(2)$

FEE CATEGORY: 1OB(2) 


\section{DOCKET\# : 07100256}

REVISIONA: 5

USER NAME: OMAHA PUBLIC POWER DISTRICT 444 SOUTH $16 T H$ STREET MALL

OMAHA

ATTN: MR. W. G. GATES

NE 68102247
EXPIRATION DATE: 07/31/1999

APPROVAL TYPE: NORMAL AND SPECIAL FORM

REGULATION: APPENDIX B TO 10 CFR PART 50 FEE CATEGORY: $10 B(2)$

\section{$\operatorname{USE}(S)$}

PROCUREMENT MAINTENANCE REPAIR

USE

COMMENTS:

DOCKETH : 07100258

REVISION\#: 9

USER NAME: AMERICAN ECOLOGY CORPORATION 109 FLINT ROAD

DAK RIDGE

ATTN: MR. ART PALMER

TN 37830
EXPIRAT ION DATE: $04 / 30 / 2000$

$01 / 14 / 1980$

APPROVAL TYPE: NORMAL AND SPECIAL FORM

REGULATION: SUBPART H TO 10 CFR PART 71 FEE CATEGORY: $10 B(2)$

COMMENTS:

DOCKETH : 07100260

REVISION\#: 4

USER NAME : ASTROTECH, INCORPORATED 7801 ALLENTOWN BLVD. HARRISBURG ATTN: MR. L. MYKYTA
PA 17112
EXPIRATION DATE: $01 / 31 / 2000$ ORIG. ISSUE DATE : 09/26/1979 APPROVAL TYPE: SPECIAL FORM

REGULATION: SUBPART H TO 10 CFR PART 71
USE(S)

PROCUREMENT MAINTENANCE REPAIR

COMMENTS: 
REVISION\# : 3

USER NAME: WISCONSIN INDUSTRIAL TESTING, INC. $4250 \mathrm{~N}$. 126TH STREET BROOKFIELD
ATTN: MR. TERRY WALLANDER WI 53005

REVISION\# : 4

DOCKET\# : 07100263

USER NAME: YUBA HEAT TRANSFER DIVISION P.0. BOX 3158

TULSA MR. JACK G. FEDERLINE ${ }^{\text {OK } 741013158}$
EXPIRATION DATE:
ORIG. ISSUE DATE: $08 / 31 / 1998$
$08 / 1979$

APPROVAL TYPE: SPECIAL FORM

REGULATION: SUBPART $H$ TO 10 CFR PART 71 FEE CATEGORY: $10 B(2)$

\section{COMMENTS :}

EXPIRATION DATE: $11 / 30 / 1999$
ORIG. I SSUE DATE : $09 / 07 / 1979$

APPROVAL TYPE: SPECIAL FORM

REGULATION: SUBPART H TO 10 CFR PART 71
FEE CATEGORY: $10 B(2)$

COMMENTS:

\section{DOCKET\#: 07100266}

REVISION\# : 5

USER NAME: DUKE POWER COMPANY

P. O. BOX 1006

CHARLOTTE M. S, TUCKMAN
NC 282011006
EXPIRATION DATE: 07/31/1999

APPROVAL TYPE: NORMAL AND SPECIAL FORM

REGULATION: APPENDIX B TO 10 CFR PART 50 FEE CATEGORY: $10 B(2)$
USE (S)

PROCUREMENT MAINTENANCE REPAIR USE

COMMENTS: 
DOCKETH : 07100269

REVISION\#: 5

USER NAME: LEHIGH TESTING LABORATORIES, INC. 308 WEST BASIN ROAD NEW CASTLE
EXPIRATION DATE: $10 / 31 / 1999$

APPROVAL TYPE: SPECIAL FORM

REGULATION: SUBPART H TO 10 CFR PART 71 FEE CATEGORY: $10 B(2)$
USE(S)

PROCUREMENT MAINTENANCE MAPATENAM USE

COMMENTS:

\section{DOCKETH : 07100275}

REVISION\#: 6

USER NAME: DUQUESNE LIGHT COMPANY BEAVER VALLEY PWR STATION PO BOX 4 ATTN: MR. R. K. BROSI

PA 150770004
EXPIRATION DATE: 08/31/1999 ORIG. ISSUE DATE: 05/07/1979

APPROVAL TYPE: NORMAL AND SPECIAL FORM

REGULATION: APPENDIX B TO 10 CFR PART 50
FEE CATEGORY: $10 B(2)$
USE (S)

PROCUREMENT

MAINTENANCE REPAIR USE

COMMENTS:

DOCKETH : 07100296

REVISION\# : 5

USER NAME: IES UTILITIES, INC.

CEDAR RAPIDS
EXPIRATION DATE:
ORIG. ISSUE DATE:
$04 / 10 / 1999$

APPROVAL TYPE: NORMAL AND SPECIAL FORM

REGULATION: APPENDIX B TO 10 CFR PART 50
USE (S)

PROCUREMENT MAINTENANCE REPAIR

\section{COMMENTS :}


DOCKETH : 07100300

REVISIONH : 5

USER NAME: INDEPENDENT TESTING LABORATORIES

19424 PARK ROW. NO. 110

HOUSTON WX 77084

COMMENTS :
EXPIRATION DATE: $11 / 30 / 1999$

APPROVAL TYPE: SPECIAL FORM

REGULATION: SUBPART H TO 10 CFR PART 71

FEE CATEGORY: $10 B(2)$
USE(S)

PROCUREMENT

MAINTENANCE

REPAIR

USE

\section{DOCKET\#: 07100301}

REVISION\#: 4

USER NAME: CTL ENGINEERING INC.

2860 F I SHER ROAD

P. O. BOX 44469

COLUMBUS 340 OH 43204

ATTN: MR. J. L. CROWLEY, RSO
EXPIRATION DATE: 04/30/2000

APPROVAL TYPE: SPECIAL FORM

REGULATION: SUBPART -H TO 10 CFR PART 71

FEE CATEGORY: $10 B(2)$
USE (S)

USE

MAINTENANCE

PROCUREMENT

REPAIR

COMMENTS :

DOCKET\# : 07100308

REVISION\# : 4

USER NAME: ALASKA INDUSTRIAL X-RAY INC. 4047 KINGSTON DRIVE

ANCHORAGE

ATTN: MS. C. A. MILLAR

AK 99504
EXPIRATION DATE: $03 / 31 / 2000$

APPROVAL TYPE: SPECIAL FORM

REGULATION: SUBPART H TO 10 CFR PART 71 FEE CATEGORY: 1OB(2)
USE(S)

PROCUREMENT MAINTENANCE REPAIR

USE

COMMENTS : 
EXPIRATION DATE: $12 / 31 / 1999$

ORIG. ISSUE DATE: 09/04/1979

APPROVAL TYPE: SPECIAL FORM

MASON \& HANGER-SILAS MASON
IOWA ARMY AMMUNITION PLANT

MIDDLETOWN

ATTN: MR. J. E. SHANNON

IA 52638

COMMENTS :

DOCKETH : 07100317

\section{REVISION\# : 4}

USER NAME: HUDSON PRODUCTS CORPORATION

BEASLEY
ATTN: MR. LESTER SRALLA, JR.

COMMENTS :

DOCKETH : 07100319

REVISION\# : 4

USER NAME: GENERAL TESTING AND INSPECTION COMPANY

\section{HIGH STREET
WASHINGTONVILLE
ATTN: MR. R. E. KRUEGEL OH 44490 \\ 780 HIGH STREET
WASHINGTONVILLE
ATTN: MR. R. E. KRUEGEL OH 44490}

REGULATION: SUBPART H TO 10 CFR PART 71
FEE CATEGORY: $10 B(2)$
$\operatorname{USE}(S)$

PROCUREMEN

MAINTENANC

REPAIR

USE

COMMENTS:

EXPIRATION DATE: $02 / 28 / 2000$
ORIG. ISSUE DATE: $07 / 19 / 1979$

APPROVAL TYPE: SPECIAL FORM

REGULATION: SUBPART H TO 10 CFR PART 71

\section{USE (S)}

PROCUREMENT

MAINTENANCE

REPAIR
EXPIRATION DATE: $01 / 31 / 2000$
ORIG. ISSUE DATE : $06 / 20 / 1979$

APPROVAL TYPE: SPECIAL FORM

REGULATION: SUBPART H TO 10 CFR PART 71
FEE CATEGORY: $10 B(2)$
$\operatorname{USE}(S)$

PROCUREMENT MAINTENANCE REPAIR
USE 
DOCKET\# : 07100325

REVISION\# : 5 USER NAME: CONSUMERS POWER COMPANY 27780 BLUE STAR HWY. ATTN: MR. THOMAS P. NEAL
EXPIRATION DATE: $11 / 30 / 1999$
ORIG. ISSUE DATE : 06/01/1979

APPROVAL TYPE: NORMAL AND SPECIAL FORM REGULATION: APPENDIX B TO 10 CFR PART 50
FEE CATEGORY: $10 B(2)$
$\operatorname{USE}(S)$

PROCUREMENT MAINTENANCE REPAIR USE

COMMENTS :
DOCKETH : 07100326

REVISION\# : 5

USER NAME: INDUSTRIAL NDT SERVICES DIVISION 2124 WENDELL AVENUE,

INDIANAPOLIS P O BOX 2245
EXPIRATION DATE: $11 / 30 / 1999$
ORIG. ISSUE DATE: $06 / 20 / 1979$

APPROVAL TYPE: SPECIAL FORM

REGULATION: SUBPART H TO 10 CFR PART 71

FEE CATEGORY: 1OB(2)

\section{USE (S)}

PROCUREMENT MAINTENANCE REPAIR USE
COMMENTS :

DOCKET\# : 07100327

REVISION\#: 7

EXPIRATION DATE: 03/31/1999

APPROVAL TYPE: NORMAL AND SPECIAL FORM

PORTLAND GENERAL ELECTRIC CO
71760 COLUMBIA RIVER HIGHWAY

PORTLAND
ATTN: MR. STEPHEN M. QUENNOZ 97048
REGULATION: APPENDIX B TO 10 CFR PART 50 FEE CATEGORY: $1 O B(1)$

\begin{tabular}{l} 
USE(S) \\
\hline ASSEMBLY \\
MODIFICATION \\
FABRICATION \\
TESTING \\
DESIGN \\
PROCUREMENT \\
MAINTENANCE \\
REPAIR \\
USE
\end{tabular}

COMMENTS: 
DOCKET\# : 07100328

REVISION\# : 4

USER NAME: PUBLIC SERVICE ELECTRIC AND GAS COMPANY

HANCOCKS BRIDGE

ATTN: MR. RUSSELL AVESON

NJ 08038

COMMENTS:

DOCKETH : 07100330

REVISION\# : 4

USER NAME: ALLIED INSPECTION SERVICES, INC.

P. 0. BOX 268

STTYN: CLAIR MR. THOMAS D. GRASHAW 48079

COMMENTS :

DOCKETH : 07100331

REVISIONH : 4

USER NAME: N.C. STATE UNIVERSITY DEPTं. OF NUCLEAR ENGINEERING RALEIGH 7909

ATTN: MR. GERALD WICKS

NC 27695
EXP IRATION DATE: $12 / 31 / 1999$

ORIG. ISSUE DATE: 06/01/1979

APPROVAL TYPE: NORMAL AND SPECIAL FORM

REGULATION: APPENDIX B TO 10 CFR PART 50
FEE CATEGORY: $1 O B(2)$
USE (S)

PROCUREMENT MAINTENANCE REPAIR

USE
EXPIRATION DATE: $01 / 31 / 2000$

APPROVAL TYPE: SPECIAL FORM

REGULATION: SUBPART H TO 10 CFR PART 71 FEE CATEGORY: $1 O B(2)$
USE (S)

USE MAINTENANCE PROCUREMENT REPAIR

\section{COMMENTS:}

EXP IRATION DATE:
ORIG. ISSUE DATE:

APPROVAL TYPE: SPECIAL FORM

REGULATION: SUBPART H TO 10 CFR PART 71

FEE CATEGORY: EDUC
USE (S)

PROCUREMENT MAINTENANCE REPAIR 
EXP IRATION DATE: $10 / 31 / 1999$
ORIG. ISSUE DATE : $06 / 29 / 1979$

APPROVAL TYPE: NORMAL AND SPECIAL FORM

FLORIDA POWER CORPORATION
$15760 \mathrm{~W}$. POWER LINE STREET

CRYSTAL RIVER

ATTN: MR. P. M. BEARD, JR.
FL 344286708 REGULATION: APPENDIX B TO 10 CFR PART 50
FEE CATEGORY: $10 \mathrm{~B}(2)$
USE(S)

PROCUREMENT

MAINTENANCE

REPAIR

USE

COMMENTS:

DOCKET\# : 07100333

REVISIONH : 6

USER NAME: SOUTHERN NUCLEAR OPERATING COMPANY, FARLEY PROJECT P. O. BOX 1295

BIRMINGHAM

COMMENTS :
REVISION\# : 6

USER NAME: GPU NUCLEAR CORPORATION, TMI

P. 0. BOX 480, ROUTE 441 SOUTH

MIDDLETOWN

PA 170570480
EXPIRATION DATE: $12 / 31 / 1999$
ORIG. ISSUE DATE: $07 / 31 / 1979$

APPROVAL TYPE: NORMAL AND SPECIAL FORM

REGULATION: APPENDIX B TO 10 CFR PART 50 FEE CATEGORY: $1 O B(2)$
$\operatorname{USE}(S)$

PROCUREMENT MAINTENANCE

REPAIR

USE

$$
\text { ATTN: MR. JOHN S. SCHORK }
$$

EXPIRATION DATE: $10 / 31 / 1999$

APPROVAL TYPE: SPECIAL FORM

REGULATION: APPENDIX B TO 10 CFR PART 50

FEE CATEGORY: $10 B(2)$
USE (S)

PROCUREMENT

MAINTENANCE

REPAIR

USE

COMMENTS : 
DOCKET\# : 07100337

\section{REVISION\#: 4}

USER NAME: CALUMET TESTING SERVICE INC. P. 0. BOX 1510 HIGHLAND ATTN: MR. T. J. KEILMAN

IN 46322

COMMENTS :
REVISION\# : 4

USER NAME: WISCONSIN CENTRIFUGAL, INC 905 EAST ST. PAUL AVENUE WAUKESHA

ATTN: MR THOMAS L. PENDER WI 531883898
EXPIRATION DATE: $02 / 28 / 2001$ APPROVAL TYPE: SPECIAL FORM

REGULATION: SUBPART H TO 10 CFR PART 71 FEE CATEGORY: $10 B(2)$
USE (S)

PROCUREMENT MAINTENANCE REPAIR USE
EXPIRATION DATE : $11 / 30 / 1999$

ORIG. I SSUE DATE: $08 / 15 / 1979$

APPROVAL TYPE: SPECIAL FORM

REGULATION: SUBPART H TO 10 CFR PART 71 FEE CATEGORY: 1 OB (2)

\begin{tabular}{l} 
USE (S) \\
\hline PROCUREMENT \\
MAINTENANCE \\
REPAIR \\
USE
\end{tabular}
REPAIR

COMMENTS :

DOCKET\#： 07100341

REVISION\#: 6

USER NAME: ENTERGY OPERATIONS, INC 1448 S.R. 333

RUSSELLVILLE

ATTN: MR. DWIGHT C. MIMS
AR 72801
EXPIRAT ION DATE: $01 / 31 / 2000$
ORIG. ISSUE DATE: $09 / 17 / 1979$

APPROVAL TYPE: NORMAL AND SPECIAL FORM

REGULATION: APPENDIX B TO 10 CFR PART 50 FEE CATEGORY: 1 OB (2)

COMMENTS: 
DOCKET\# : 07100345

REVISION\#: 7

USER NAME: CAROLINA POWER AND LIGHT COMPANY 411 FAYETTEVILLE STREET MALL. P. 0 BOX 1551

ATTN: MR. R. E. ROGAN

NC 276021551
EXPIRATION DATE: $11 / 30 / 1999$

APPROVAL TYPE: NORMAL AND SPECIAL FORM

REGULATION: APPENDIX B TO 10 CFR PART 50 FEE CATEGORY: $10 B(1)$
$\operatorname{USE}(S)$

DESIGN FABRICATION

ASSEMBLY

TESTING

PROCUREMENT

MAINTENANCE

REPAIR

MOD IFICATION

USE

COMMENTS :

DOCKET\# : 07100346

REVISION\#: 7

EXPIRATION DATE: $08 / 31 / 2000$

ORIG. ISSUE DATE: 09/17/1979

USER NAME: PUBLIC SERVICE COMPANY OF COLORADO

16805 WELD COUNTY ROAD $19-1 / 2$

PLATTEVILLE
ATTN: MR. FREDERICK J. BORSTO 80651 .

APPROVAL TYPE: NORMAL AND SPECIAL FORM

REGULATION: APPENDIX 8 TO 10 CFR PART 50 FEE CATEGORY: $1 O B(1)$

\begin{tabular}{l} 
USE(S) \\
\hline DESIGN \\
FABRICATION \\
ASSEMBLY \\
TESTING \\
PROCUREMENT \\
MAINTENANCE \\
REPAIR \\
MODIFICATION \\
USE
\end{tabular}

COMMENTS:

DOCKET\# : 07100347

REVISION\# : 4

EXP IRATION DATE : $12 / 31 / 1996$

APPROVAL TYPE: SPECIAL FORM

STONE \& WEB STER
P.O. BOX 23255
245 SUMMER STREE

$245^{\circ}$ SUMMER STREET MA 02107

ATTN: MS. CLAIRE DOHERTY

COMMENTS :
REGULATION: SUBPART H TO 10 CFR PART 71 FEE CATEGORY: $10 B(2)$
USE(S)

US

MAINTENANCE PROCUREMENT REPAIR 
REVISION\#: 6

USER NAME: UNIVERSITY OF MICHIGAN, FORD NUCLEAR REACTOR PHOENIX MEMORIAL LAB. 2301 BONISTEEL BLVD. ANN ARBOR
ATTN: MR. RONALD F. FLEMING
MI 481092100
DOCKETH : 07100352

REVISION\#: 4

USER NAME: NILES STEEL TANK COMPANY 713 WAYNE STREET, P. O. BOX 728 ATTN: MR. ROBERT H. WOOD
EXPIRATION DATE: 06/30/2000 ORIG. ISSUE DATE:

APPROVAL TYPE: NORMAL AND SPECIAL FORM

REGULATION: SUBPART H TO 10 CFR PART 71 FEE CATEGORY: EDUC
USE (S)

PROCUREMENT MAINTENANCE REPAIR

USE
COMMENTS :
DOCKETH : 07100354

REVISION\#: 8

USER NAME: ADVANCED MEDICAL SYSTEMS, INC. 121 NORTH EAGLE STREET

GENEVA

ATTN: MS. SHERRY J. STEIN
OH 44041
EXPIRATION DATE: $03 / 31 / 2000$

ORIG. ISSUE DATE: 12/18/1979

APPROVAL TYPE: SPECIAL FORM

REGULATION: SUBPART H TO 10 CFR PART 71
FEE CATEGORY: 1 IOB(2)

\section{USE(S)}

PROCUREMENT MAINTENANCE REPAIR USE
MI 49120
EXPIRATION DATE: $01 / 31 / 2000$

APPROVAL TYPE: NORMAL AND SPECIAL FORM

REGULATION: SUBPART H TO 10 CFR PART 71 FEE CATEGORY! $1 O B(1)$

\begin{tabular}{l} 
USE(S) \\
\hline ASSEMBLY \\
MODIFICATION \\
FABRICATION \\
TESTING \\
DESIGN \\
PROCUREMENT \\
MAINTENANCE \\
REPAIR \\
USE
\end{tabular}

COMMENTS : 
EXP IRATION DATE: $02 / 28 / 2000$
ORIG. ISSUE DATE: $10 / 23 / 1979$

APPROVAL TYPE: SPECIAL FORM

GPU NUCLEAR CORP . , OYSTER CREEK

P. O. BOX 388

ROUTE 9 SOUTH

ATTN: MR. ROBERT HILLMAN

NJ 170570480

COMMENTS :

DOCKET\# : 07100362

REVISION\#: 5

USER NAME: WISCONSIN PUBLIC SERVICE CORPORATION 600 NORTH ADAMS GREF BOX 19002

DOCKET\# : 07100363

REVISION\# : 4

USER NAME: BRIGGS ASSOCIATES, INC.

400 HINGHAM STREET

\section{ROCKLAND
ATTN: MR. PAUL M. SKOROHOD MA 02370 \\ ROCKLAND}

REGULATION: APPENDIX B TO 10 CFR PART 50
FEE CATEGORY: $10 B(2)$
USE $(S)$

PROCUREMENT

MAINTENANCE

REPAIR
USE

COMMENTS :

EXPIRATION DATE : $03 / 31 / 2000$
ORIG. ISSUE DATE : $12 / 17 / 1979$

APPROVAL TYPE: NORMAL AND SPECIAL FORM

REGULATION: APPENDIX B TO 10 CFR PART 50
FEE CATEGORY: $10 B(2)$
USE (S)

PROCUREMENT

MAINTENANCE

REPAIR
EXPIRATION DATE: $03 / 31 / 2000$

ORIG. ISSUE DATE: $12 / 21 / 1979$

APPROVAL TYPE: SPECIAL FORM

REGULATION: SUBPART H TO 10 CFR PART 71
FEE CATEGORY: $10 B(2)$
USE (S)

PROCUREMENT

MAINTENANCE

REPAIR

USE 
EXPIRATION DATE: 06/30/2000

ORIG. ISSUE DATE : 07/29/1980

BRANCH RADIOGRAPHIC LABS. , INC.

32 SOUTH AVENUE W

CRANFORD

ATTN: MR. PETER J. BRANCH

NJ 07016
APPROVAL TYPE: SPECIAL FORM

REGULATION: SUBPART H TO 10 CFR PART 71 FEE CATEGORY: 1OB(2)

\section{USE (S)}

PROCUREMENT MAINTENANCE REPAIR

COMMENTS:

DOCKET\# : 07100377

\section{REVISION\# : 5}

USER NAME: INDIANA MICHIGAN POWER COMPANY P. 0. BOX 16631

COLUMBUS

ATTN: MR. E. E. FITZPATRICK ${ }^{\text {OH }} 43216$
EXP IRATION DATE : $06 / 30 / 2000$

ORIG. ISSUE DATE: $03 / 27 / 1980$

APPROVAL TYPE: NORMAL AND SPECIAL FORM REGULATION: APPENDIX B TO 10 CFR PART 50
FEE CATEGORY: $10 \mathrm{OB}(2)$

\section{USE (S)}

PROCUREMENT MAINTENANCE REPAIR

USE

COMMENTS:

DOCKETH : 07100378

REVISION\#: 7

USER NAME: WISCONSIN ELECTRIC POWER CO. 231 WEST MICHIGAN, MILWAUKEE 2046 ATTN: MR. C. W. FAY
WI 53201
EXPIRATION DATE: $07 / 31 / 2000$

APPROVAL TYPE: NORMAL AND SPECIAL FORM

REGULATION: APPENDIX B TO 10 CFR PART 50 FEE CATEGORY: $10 B(2)$
USE(S)

PROCUREMENT MAINTENANCE REPAIR

COMMENTS : 
REVISION\# : 4

USER NAME: GENERAL ELECTRIC COMPANY P. 0. BOX 460, VALLECITOS ROAD VALLECITOS NUCLEAR CENTER PLEASANTON
ATTN: MR. G. E. CUNNINGHAM CA 94566
EXPIRATION DATE: $11 / 30 / 2000$
ORIG. ISSUE DATE : $03 / 31 / 1980$

APPROVAL TYPE: NORMAL AND SPECIAL FORM

REGULATION: APPENDIX B TO 10 CFR PART 50 FEE CATEGORY: $1 O B(1)$

\section{USE (S)}

\section{DESIGN}

FABRICATION

ASSEMBLY

TESTING

PROCUREMENT

MAINTENANCE

REPAIR

MODIFICATION

USE

COMMENTS :

\section{DOCKET\# : 07100383}

REVISION\# : 5

USER NAME: BALTIMORE GAS AND ELECTRIC COMPANY CALVERT CLIFFS NUC. PWR. PLANT 1650 CLAVERT CLIFFS PARKWAY

LUSBY

ATTN: MR. R. E. DENTON

COMMENTS :
REVISION\# : 4

USER NAME: DEPARTMENT OF COMMERCE. NIST

GAITHERSBURG

ATTN: MR. LYMAN E. PEVEY
DOCKET\#: 07100390
MD 20899
EXPIRATION DATE: $10 / 31 / 2000$

ORIG. ISSUE DATE: $07 / 15 / 1980$

APPROVAL TYPE: NORMAL AND SPECIAL FORM

REGULATION: APPENDIX B TO 10 CFR PART 50 FEE CATEGORY: $10 B(2)$

\section{USE (S)}

PROCUREMENT MAINTENANCE REPAIR USE
EXPIRATION DATE: $09 / 30 / 2000$

ORIG. ISSUE DATE : 06/12/1980

APPROVAL TYPE: SPECIAL FORM

REGULATION: SUBPART H TO 10 CFR PART 71 FEE CATEGORY: $10 B(2)$

\section{USE (S)}

PROCUREMENT MAINTENANCE REPAIR USE 
REVISION\# : 4

USER NAME: BAKER TESTING SERVICES, INC. 98 RESERVOIR PARK DRIVE ROCKLAND
ATTN: MR. DONALD PALMER, RSO 02370 COMMENTS :

DOCKET\# : 07100393

USER NAME: VOITH HYDRO INC.

YORK

ATTN: MR. HAROLD LILLICH

\section{REVISION\#: 6}

PA 17405

COMMENTS :

\section{DOCKET\# : 07100404}

USER NAME: CTI ALASKA, INC.

4831 OLD SÉWARD HWY, SUITE 107

\section{ANCHORAGE ATTN: MR. SANDY N. WATSON \\ AK 99503}

REVISIONH : 5
EXPIRATION DATE: $02 / 28 / 2001$
ORIG. ISSUE DATE: $07 / 07 / 1980$

APPROVAL TYPE: SPECIAL FORM

REGULATION: SUBPART H TO 10 CFR PART 71
FEE CATEGORY: $10 B(2)$
USE (S)

PROCUREMENT

MAINTENANCE

REPAIR

USE

COMMENTS :

EXPIRATION DATE: 02/28/2001

ORIG. ISSUE DATE: 07/01/1980

APPROVAL TYPE: SPECIAL FORM

REGULATION: SUBPART H TO 10 CFR PART 71 FEE CATEGORY: $10 B(2)$
USE (S)

PROCUREMENT MAINTENANCE REPAIR MODIFICATION USE
EXPIRATION DATE: 04/30/2001

ARIG. ISSUE DATE: O1/28/1981

APPROVAL TYPE: SPECIAL FORM

REGULATION: SUBPART H TO 10 CFR PART 71 FEE CATEGORY: $1 O B(2)$

\begin{tabular}{l} 
USE (S) \\
\hline PROCUREMENT \\
MAINTENANCE \\
REPAIR \\
USE
\end{tabular}


DOCKETH : 07100408

REVISION\# : 4

USER NAME: MISSOURI STEEL CASTINGS COMPANY $905 \mathrm{E} .3 \mathrm{RD}$ ST. JOP 0 iN ATTN: MR. DAVID GUTHRIE

MO 64802
EXPIRAT ION DATE: $03 / 31 / 2001$

APPROVAL TYPE: SPECIAL FORM

REGULATION: SUBPART H TO 10 CFR PART 71 FEE CATEGORY: 1OB(2)
USE(S)

PROCUREMENT MAINTENANCE

REPAIR

USE

COMMENTS :

DOCKETH : 07100417

REVISION\# : 4

USER NAME: NDT SERVICES, INC

P. 0. BOX 4952, SUITE 370

CAGUAS PR 007264952

ATTN: MR. RODNEY LILLARD
EXPIRATION DATE: $01 / 31 / 1999$

APPROVAL TYPE: SPECIAL FORM

REGULATION: SUBPART H TO 10 CFR PART 71 FEE CATEGORY: $10 B(2)$

COMMENTS:

DOCKETH : 07100420

\section{REVISION\# : 7}

USER NAME: RTI INC PROCESS TECHNOLOGY SUBSIDIARIES

108 LAKE DENMARK ROAD

ROCKAWAY JOHN D. SCHLECHT NJ 07866
EXP IRATION DATE: $04 / 30 / 2001$

ORIG. ISSUE DATE : 04/06/1981

APPROVAL TYPE: SPECIAL FORM

REGULATION: SUBPART H TO 10 CFR PART 71 FEE CATEGORY: $10 B(2)$
USE (S)

USE PROCUREMENT REPAIR

\section{COMMENTS :}




\section{DOCKETH: 07100422}

REVISION\#: 4

USER NAME: AMERICAN FOUNDRY GROUP INC. $1200 \mathrm{~N}$. PEORIA AVENUE

TULSA MR. CARL CRUCE

OK 74106
EXPIRATION DATE: $07 / 31 / 2001$
ORIG. ISSUE DATE : 04/06/1981 APPROVAL TYPE: SPECIAL FORM

REGULATION: SUBPART H TO 10 CFR PART 71 FEE CATEGORY: $10 B(2)$
USE(S)

PROCUREMENT MAINTENANCE REPAIR

USE

COMMENTS :

\section{DOCKETH : 07100423}

REVISION\#: 5

USER NAME: $H \& H X$ \& $H A Y$ SERVICES, INC.

110 BARR LANE

WEST MONROE 51

ATTN: MR. K. Y. HEAD, RSO

LA 712940517
EXPIRATION DATE: 04/30/2001

ORIG. ISSUE DATE: 04/08/1981

APPROVAL TYPE: SPECIAL FORM

REGULATION: SUBPART H TO 10 CFR PART 71 FEE CATEGORY: $1 O B(2)$

\begin{tabular}{l} 
USE(S) \\
\hline PROCUREMENT \\
MAINTENANCE \\
REPAIR \\
USE
\end{tabular}

COMMENTS :

DOCKETH: 07100428

REVISION\# : 4

USER NAME: BILL MILLER INC.

HENRYETTA $W$. A. MILLER OK 74437
EXP IRATION DATE: 06/30/2001

ORIG. ISSUE DATE : 04/15/1981

APPROVAL TYPE: SPECIAL FORM

REGULATION: SUBPART H TO 10 CFR PART 71
FEE CATEGORY: $10 B(2)$
USE(S)

PROCUREMENT

MAINTENANCE

REPAIR

COMMENTS: 
DOCKETH : 07100429

REVISIONH : 4 USER NAME: $\begin{aligned} & \text { QC LABORATORIES, INC. } \\ & 2870 \text { STIRLING ROAD }\end{aligned}$

\section{HOLLYWOOD}

ATTN: MR. R. D. STICKLER

FL 330201199

COMMENTS:

DOCKETH : 07100437

REVISION\# : 4

USER NAME: ADVEX CORP.

121 FLOYD THOMPSON DRIVE

HAMPTON

ATTN: MR. G. E. SLEMP

VA 236661307

COMMENTS :

DOCKETH : 07100438

REVISION\# : 4

USER NAME: GENERAL MOTORS POWERTRAIN DIVISION DEF IANCE PLANT

ATTN: MR. MICHAEL PREZKOP

$\mathrm{OH} 43512$

DEF IANCE PLANT
DEFIANCE. MICHAEL PREZKOP OH 43512
ATTN: MR. MIC

EXPIRATION DATE: 05/31/2001

ORIG. ISSUE DATE: 04/15/1981

APPROVAL TYPE: SPECIAL FORM

REGULATION: SUBPART H TO 10 CFR PART 71

FEE CATEGORY: $10 B(2)$
USE (S)

PROCUREMENT

MAINTENANCE

REPAIR

MODIFICATION

EXPIRATION DATE: 07/31/2001

APPROVAL TYPE: SPECIAL FORM

REGULATION: SUBPART H TO 10 CFR PART 71
FEE CATEGORY: $10 B(2)$

\begin{tabular}{l} 
USE $(S)$ \\
\hline USE \\
PROCUREMENT \\
MAINTENANCE \\
REPAIR
\end{tabular}

\section{COMMENTS :}

EXPIRATION DATE: $06 / 30 / 2001$
ORIG. ISSUE DATE: $04 / 21 / 1981$

APPROVAL TYPE: SPECIAL FORM

REGULATION: SUBPART H TO 10 CFR PART 71
FEE CATEGORY: $10 B(2)$

\begin{tabular}{l} 
USE $(S)$ \\
\hline USE \\
PROCUREMENT \\
MAINTENANCE \\
REPAIR
\end{tabular}




\section{DOCKETH: 07100440}

REVISIONH : 4

USER NAME: PROFESSIONAL SERVICE INDUSTRIES, INC. 510 EAST 22ND STREET

LOMBARD ${ }_{\text {ATR. DAVID S. PRICE }}$ IL 60148

COMMENTS :
REVISION\# : 3

USER NAME: NATIONAL INSPECTION \& CONSULTANTS, INC. 3949 EVANS AVE.

FORT MEYERS

FL 33901
EXPIRATION DATE: $08 / 31 / 2001$

APPROVAL TYPE: SPECIAL FORM REGULATION: SUBPART H TO 10 CFR PART 71
FEE CATEGORY: $10 B(2)$
USE(S)

PROCUREMENT MAINTENANCE REPAIR USE

\section{COMMENTS :}

DOCKETH: 07100460

REVISION\# : 4

USER NAME: UNIVERSITY OF VIRGINIA, NUCLEAR REACTOR FACILITY DEPT. OF NUCLEAR ENGINEERING

CHARLOTTESVILLE ATTN: MR. J.P. FARRAR
EXPIRATION DATE: $12 / 31 / 1996$

ORIG. ISSUE DATE: $11 / 24 / 198$

APPROVAL TYPE: SPECIAL FORM

REGULATION: SUBPART H TO 10 CFR PART 71 FEE CATEGORY: $10 B(2)$
USE (S)

PROCUREMENT MAINTENANCE REPAIR USE

COMMENTS:

EXP IRATION DATE: 09/30/1997 ORIG. ISSUE DATE:

APPROVAL TYPE: SPECIAL FORM

REGULATION: APPENDIX B TO 10 CFR PART 50 FEE CATEGORY: EDUC
USE(S)

PROCUREMENT MAINTENANCE REPAIR REPA 
DOCKET\# : 07100465

REVISION\# : 4

USER NAME: MAINE YANKEE ATOMIC POWER CO. 329 BATH ROAD

BRUNSWICK

ATTN: MR. JAMES R. HEBERT

ME 04011
EXPIRATION DATE: 08/31/2001

ORIG. ISSUE DATE : 11/10/1981

APPROVAL TYPE: NORMAL AND SPECIAL FORM

REGULATION: APPENDIX B TO 10 CFR PART 50

FEE CATEGORY: $10 B(2)$
USE (S)

PROCUREMENT

MAINTENANCE

REPAIR

COMMENTS:

DOCKETH : 07100468

REVISION\#: 3

USER NAME: EL PASO NATURAL GAS COMPANY

P. 0. BOX 1492

ELPASO AR. E. J. MARCOUX TX 79978

COMMENTS:

DOCKET\#： 07100470

\section{REVISION\#: 3}

USER NAME: PENNSYLVANIA STATE UNIVERSITY 207 OLD MAIN

UNIVERSITY PARK

ATTN: MR. DAVID A. SHIRLEY
PA 168021503
EXPIRATION DATE: 07/31/1998

ORIG. ISSUE DATE: $01 / 28 / 1982$

APPROVAL TYPE: SPECIAL FORM

REGULATION: SUBPART H TO 10 CFR PART 71

FEE CATEGORY: 1OB(2)

\begin{tabular}{l} 
USE (S) \\
\hline USE \\
MAINTENANCE \\
PROCUREMENT \\
REPAIR
\end{tabular}

EXPIRATION DATE: 10/31/1996

ORIG. ISSUE DATE:

APPROVAL TYPE: NORMAL AND SPECIAL FORM

REGULATION: SUBPART H TO 10 CFR PART 71 FEE CATEGORY: EDUC

COMMENTS : 


\section{DOCKET\#: 07100471}

REVISION\#: 4

USER NAME: PENNSYLVANIA POWER AND LIGHT COMPANY

TWO NORTH NINTH STREET

ALLENTOWN

COMMENTS:

DOCKETH : 07100472

\section{REVISION\#: 3}

USER NAME: ABC TESTING INC.

95 FIRST STREET

BRIDGEWATER

ATTN: MR. C. A. RICHARDSON MA 02324
EXPIRATION DATE:
ORIG. ISSUE DATE : 04/30/2001
OR/26/1982

APPROVAL TYPE: NORMAL AND SPECIAL FORM

REGULATION: APPENDIX B TO 10 CFR PART 50 FEE CATEGORY: $10 B(2)$

\section{$\operatorname{USE}(S)$}

PROCUREMENT MAINTENANCE REPAIR

USE
DOCKETH : 07100476

REVISION\#: 5 USER NAME: MIDWEST INSPECTION SERVICES, INC.
514 SOUTH MAIN, P.O. BOX 1229 PERRYTON ATTN: MR. RAYMOND EZZELL
TX 79070
EXPIRATION DATE: 05/31/1997 ORIG. I SSUE DATE: 05/10/1982 APPROVAL TYPE: SPECIAL FORM REGULATION: SUBPART H TO 10 CFR PART 71
FEE CATEGORY: $10 B(2)$ EXPIRATION DATE: $01 / 31 / 1997$
ORIG. ISSUE DATE: $01 / 19 / 1983$ APPROVAL TYPE: SPECIAL FORM REGULATION: SUBPART H TO 10 CFR PART 71
USE (S)

PROCUREMENT MAINTENANCE REPAIR

COMMENTS: 


\section{DOCKETH : 07100479}

REVISION\#: 4

USER NAME: SOUTH CAROLINA ELECTRIC \& GAS CO. P. 0. BOX 88

JENKINSVILLE

ATTN: MR. JOHN L. SKOLDS

SC 29065

COMMENTS :

DOCKET\# : 07100482

REVISION\# : 3

USER NAME: UNIVERSITY OF MASSCHUSETTS N414 MORRILL SCIENCE CENTER

AMHERST JIM TOCCI MA 01003

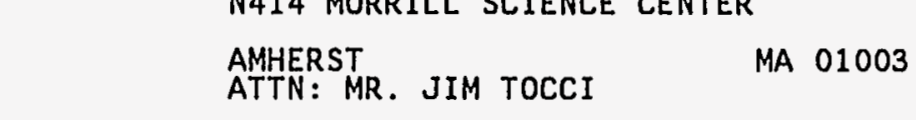

EXP IRATION DATE: $07 / 31 / 1997$

APPROVAL TYPE: NORMAL AND SPECIAL FORM

REGULATION: APPENDIX B TO 10 CFR PART 50

REGULATION: APPENDIX
FEE CATEGORY: $10 B(2)$
USE (S)

PROCUREMENT

MAINTENANCE

REPAIR

USE

COMMENTS:

DOCKET\# : 07100484

REVISION\# : 4

USER NAME: NEBRASKA PUBLIC PWR DISTRICT P.O. BOX 98

BROWNVILLE

ATTN: MR. J. H. MUELLER
NE 68321
EXPIRATION DATE: $08 / 31 / 1997$
ORIG. ISSUE DATE:

APPROVAL TYPE: SPECIAL FORM REGULATION: SUBPART H TO 10 CFR PART 71
FEE CATEGORY: EDUC
USE (S)

PROCUREMENT MAINTENANCE REPAIR USE

\section{COMMENTS :}

EXP IRATION DATE: $11 / 30 / 1997$
ORIG. ISSUE DATE: $12 / 10 / 1982$

APPROVAL TYPE: NORMAL AND SPECIAL FORM

REGULATION: APPENDIX B TO 10 CFR PART 50
FEE CATEGORY: $10 B(2)$
USE(S)

PROCUREMENT MAINTENANCE REPAIR 
DOCKET\# : 07100485

REVISIÓNH: 4

USER NAME: WASH. PUB. PWR SUPPLY SYSTEM

P. 0. BOX 968

3000 GEORGE WASHINGTON WAY

RICHLAND.

WA 99352

ATTN: MR. J. V. PARRISH

COMMENTS :

DOCKET\# : 07100487

REVISION\# : 4

USER NAME: DEPARTMENT OF THE ARMY

ATTN: STEWS-DES-R (RPO)

WHITE SANDS MISSILE RANGE NM 880025048 COMMANDER

DOCKETH : 07100489

REVISION\#: 5

USER NAME: SACRAMENTO MUNICIPAL UTILITY DISTRICT

6201 S STREET, P. 0. BOX 15830

SACRAMENTO ATTN: MR. JAMES R. SHETLER

55821830

COMMENTS:
EXPIRATION DATE: $11 / 30 / 1997$
ORIG. ISSUE DATE: $11 / 09 / 1982$

APPROVAL TYPE: NORMAL AND SPECIAL FORM

REGULATION: APPENDIX B TO 10 CFR PART 50

FEE CATEGORY: $1 O B(2)$
USE (S)

PROCUREMENT MAINTENANCE REPAIR

USE
EXPIRATION DATE: $12 / 31 / 1997$

APPROVAL TYPE: SPECIAL FORM

REGULATION: SUBPART H TO 10 CFR PART 71

FEE CATEGORY: $10 B(2)$
USE (S)

PROCUREMENT

MAINTENANCE

REPAIR

USE
EXPIRATION DATE: $01 / 31 / 1998$

APPROVAL TYPE: SPECIAL FORM

REGULATION: APPENDIX B TO 10 CFR PART 50 FEE CATEGORY: $10 B(2)$
USE ( $S$ )

PROCUREMENT

MAINTENANCE

REPAIR

USE 
REVISION\#: 3

USER NAME: MONTANA X-RAY INC.
P.O.BOX 50183

BILLINGS

COMMENTS :

DOCKET\# : 07100495

\section{REVISION\#: 3}

USER NAME: SOUTHWEST X-RAY CORPORATION

P. 0. BOX 24

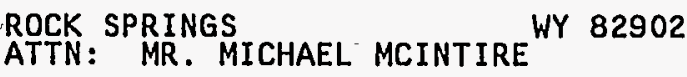

COMMENTS :

DOCKET\# : 07100496

\section{REVISION\#: 8}

USER NAME: SCIENTIFIC ECOLOGY GROUP, INC.
1560 BEAR CREEK ROAD, P. BOX 2530
ATTN: MR. J. F. MCCARTER
TN 37831 J. F. MCCARTER

EXPIRATION DATE: $04 / 30 / 1998$

APPROVAL TYPE: SPECIAL FORM

REGULATION: SUBPART H TO 10 CFR PART 71 REGULATION:
FEE CATEGORY: $10 B(2)$

\section{$\operatorname{USE}(S)$}

PROCUREMENT MAINTENANCE REPAIR USE
EXPIRATION DATE: $11 / 30 / 1999$
ORIG. ISSUE DATE: $05 / 03 / 1983$

APPROVAL TYPE: SPECIAL FORM

REGULATION: SUBPART H TO 10 CFR PART 71 FEE CATEGORY: $10 B(2)$ \begin{tabular}{l} 
USE(S) \\
\hline USE \\
MAINTENANCE \\
PROCUREMENT
\end{tabular} REPAIR
EXPIRATION DATE: $08 / 31 / 1998$
ORIG. ISSUE DATE: $08 / 11 / 1983$

APPROVAL TYPE: NORMAL AND SPECIAL FORM

REGULATION: SUBPART H, TO 10 CFR PART 71
FEE CATEGORY: $10 B(1)$
$\operatorname{USE}(\mathrm{S})$

DESIGN FABRICATION ASSEMBLY TESTING MAINTENANCE REPAIR MODIFICATION

COMMENTS : 
EXP IRATION DATE: $06 / 30 / 1998$
ORIG. ISSUE DATE : $06 / 13 / 1983$

APPROVAL TYPE: SPECIAL FORM

HALLIBURTON LOGGING SERVICES
P. O. BOX 42800 HOUSTON ATTN: MR. JEFF PETTIGREW

TX 772422800

COMMENTS :

DOCKETH : 07100501

REVISION\#: 3 USER NAME: SCIENTIFIC INSPECTION TECHNOLOGY, INC. HIXSON ATTN: MR. DAVID W. IVEY

TN 37343

COMMENTS :

DOCKETH : 07100503

REVISION\# : 3

USER NAME: BIG STATE X-RAY, INC.

P. 0. BOX 520 CHICKASHA MR. WADE PENN

OK 73023 REGULATION: SUBPART H TO 10 CFR PART 71
FEE CATEGORY: $10 B(2)$
USE(S)

PROCUREMENT MAINTENANCE REPAIR

USE
EXPIRATION DATE: $03 / 31 / 1999$

APPROVAL TYPE: SPECIAL FORM

REGULATION: SUBPART H TO 10 CFR PART 71 FEE CATEGORY: $10 B(2)$

\section{USE(S)}

USE

MAINTENANCE

PROCUREMENT

REPAIR
EXPIRATION DATE: $10 / 31 / 1998$

APPROVAL TYPE: SPECIAL FORM

REGULATION: SUBPART H TO 10 CFR PART 71
USE(S)

PROCUREMENT

MAINTENANCE

REPAIR

REPA

COMMENTS: 
DOCKETH : 07100504

REVISION\#: 4

USER NAME: TU ELECTRIC

$400 \mathrm{~N}$. OLIVE STREET L.B. 81

DALLAS MR. WILLIAM J. CAHILL 75201

COMMENTS :

DOCKET\#：07100509

REVISION\# : 3

USER NAME: MET-CHEM TESTING LABS

369 WEST GREGSON AVE.

SALT LAKE CITY
ATTN: MR. N. WAYNE HANSEN UT 841153440
EXPIRATION DATE: $02 / 28 / 1999$

ORIG. ISSUE DATE: 10/18/1983

APPROVAL TYPE: SPECIAL FORM

REGULATION: APPENDIX B TO 10 CFR PART 50

FEE CATEGORY: $10 B(2)$
USE(S)

PROCUREMENT MAINTENANCE REPAIR USE COMMENTS:

DOCKETH : 07100514

REVISION\#: 3

USER NAME: NORTH AMERICAN INSPECTION, INC. 5260 WEST COPLAY ROAD

WHITEHALL

ATTN: MR. DON B. SHUMWAY
EXPIRATION DATE: $12 / 31 / 1998$

APPROVAL TYPE: SPECIAL FORM REGULATION: SUBPART:H TO 10 CFR PART 71
FEE CATEGORY: $10 B(2)$
USE (S)

PROCUREMENT MAINTENANCE REPAIR USE

COMMENTS:

EXPIRATION DATE: $03 / 31 / 1999$
ORIG. I SSUE DATE: $02 / 24 / 1984$

APPROVAL TYPE: SPECIAL FORM

REGULATION: SUBPART H TO 10 CFR PART 71 REGULATION: SUBPART
FEE CATEGORY: $10 B(2)$
$\operatorname{USE}(S)$

PROCUREMENT MAINTENANCE REPAIR USE 
REVISION\# : 6

USER NAME: LONG ISLAND POWER AUTHORITY SHOREHAM NUCLEAR PWR O BOX 628, NORTH COUNTRY RD

WADING RIVER ATTN: MR. L. M. HILL

COMMENTS :

DOCKETH : 07100520

REVISION\#: 4

USER NAME: UNION ELECTRIC COMPANY P. 0. BOX 149 CHOUTEAU AVENUE ATTN: MR. DONALD F. SCHNELL MO 63166

COMMENTS:
EXPIRATION DATE: $09 / 30 / 2000$
ORIG. ISSUE DATE: $04 / 16 / 1984$

APPROVAL TYPE: SPECIAL FORM

REGULATION: APPENDIX B TO 10 CFR PART 50 FEE CATEGORY: $1 O B(2)$
USE(S)

PROCUREMENT MAINTENANCE REPAIR

USE

\section{DOCKETH : 07100521}

REVISIONH: 3

USER NAME: GEORGIA POWER CO.. PLANT E. I. HATCH

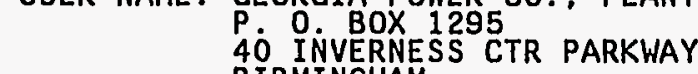 \\ P. O BOX 1295 TIR PARKWAY
40 INVERNESS CTR PAT BIRMINGHAM
ATTN: MR. W. G. HAIRSTON, III 35201}

EXP IRAT ION DATE: 06/30/1999 ORIG. ISSUE DATE: 06/13/1984

APPROVAL TYPE: SPECIAL FORM REGULATION: APPENDIX B TO 10 CFR PART 50
FEE CATEGORY: $10 B(2)$
USE(S)
PROCUREMENT
MAINTENANCE

REPAIR

USE

COMMENTS:

EXPIRATION DATE: $05 / 31 / 1999$
ORIG. ISSUE DATE: $06 / 04 / 1984$

APPROVAL TYPE: NORMAL AND SPECIAL FORM

REGULATION: APPENDIX B TO 10 CFR PART 50
USE (S)

PROCUREMENT MAINTENANCE REPAIR 
NRC-1265006-006

U.S. NUCLEAR REGULATORY COMMISSION

DOCKETH : 07100524

REVISION\#: 4

USER NAME: STERIGENICS INTERNATIONAL 4020 CLIPPER COURT

FREMONT ATTN: DR. BARRY P. FAIRAND
EXPIRATION DATE: $07 / 31 / 2000$

APPROVAL TYPE: SPECIAL FORM

CA 94538

REGULATION: SUBPART H TO 10 CFR PART 71
FEE CATEGORY: $10 B(2)$

USE(S)

PROCUREMENT MAINTENANCE MAINTENANCE USE.

COMMENTS :

DOCKETH : 07100525

REVISION\#: 3

USER NAME: PECO ENERGY COMPANY NUCLEAR GROUP HEADQUARTERS 965 CHESTERBROOK BOULLEVARD

WAYNE

ATTN: MR. G. A. HUNGER

PA 190875691
EXP IRATION DATE: $03 / 31 / 2000$

ORIG. ISSUE DATE: 02/19/1985

APPROVAL TYPE: NORMAL AND SPECIAL FORM

REGULATION: SUBPART H TO 10 CFR PART 71 FEE CATEGORY: $1 O B(2)$

COMMENTS :

DOCKETH : 07100526

REVISION\#: 3

USER NAME: DETROIT EDISON COMPANY, FERMI 2 6400 NORTH DIXIE HIGHWAY

NEWPORT

ATTN: MR. ROBERT MCKEON

MI 48166
EXP IRATION DATE: $01 / 31 / 2000$

ORIG. ISSUE DATE: 01/22/1985

APPROVAL TYPE: NORMAL AND SPECIAL FORM

REGULATION: APPENDIX B TO 10 CFR PART 50 FEE CATEGORY: $10 B(2)$
USE(S)

PROCUREMENT MAINTENANCE USEAIR

COMMENTS: 
REVISION\# : 2 USER NAME: THERAPY SERVICES INC. FREDERICK ATTN: MR. J. L. FOGLE

MD 21703 COMMENTS:
REVISION\#: 2

USER NAME: WESTERN X-RAY 3803 BRANSON ROAD POTEAU OK 74953 ATTN: MR. JAMES E. PETERS
EXPIRATION DATE: $03 / 31 / 2000$ ORIG. ISSUE DATE: 03/11/1985 APPROVAL TYPE: SPECIAL FORM

REGULATION: SUBPART H TO 10 CFR PART 71 FEE CATEGORY: $10 B(2)$
USE(S)

PROCUREMENT MAINTENANCE

USE

\section{COMMENTS:}

DOCKETH : 07100536

REVISION\# : 6

USER NAME: ENTERGY OPERATIONS INC.

P. O. BOX 756

PORT GIBSON

ATTN: MR. MARK L. CARVER
MS 39150
EXPIRATION DATE: $05 / 31 / 2000$
ORIG. I SSUE DATE: $05 / 09 / 1985$

APPROVAL TYPE: SPECIAL FORM REGULATION: SUBPART H TO 10 CFR PART 71
FEE CATEGORY: $10 B(2)$
USE (S)

PROCUREMENT MAINTENANCE REPAIR
USE

COMMENTS :

EXPIRATION DATE: $04 / 30 / 2000$
ORIG. ISSUE DATE: $04 / 23 / 1985$

APPROVAL TYPE: NORMAL AND SPECIAL FORM

REGULATION: APPENDIX B TO 10 CFR PART 50 FEE CATEGORY: $1 O B(2)$
USE(S)

PROCUREMENT MAINTENANCE REPAIR USE 
DOCKETH : 07100538

REVISIONH: 2

USER NAME: WALASHEK INDUSTRIES

G07 ALA MOANA BLVD., PIER 1

HONOLULU CARL K. YAMAMOTO HI 96813
EXP IRATION DATE: $10 / 31 / 2000$

APPROVAL TYPE: SPECIAL FORM

REGULATION: SUBPART H TO 10 CFR PART 71

REGULATION: SUBPART
FEE CATEGORY: $10 B(2)$

\section{USE(S)}

PROCUREMENT MAINTENANCE REPAIR

COMMENTS:

DOCKETH : 07100539

REVISION\# : 1

USER NAME: ARROW NDE COMPANY INC.

11307 CORTEZ DRIVE

BROKEN ARROW

COMMENTS :

DOCKETH : 07100541

REVISION\# : 4

USER NAME: PERRY NUCLEAR POWER PLANT

10 CENTER ROAD

PERRY
MGR., REGULATORY AFFAIRS SECT.

COMMENTS:
EXPIRATION DATE: 06/30/1997

APPROVAL TYPE: SPECIAL FORM

REGULATION: SUBPART H TO 10 CFR PART 71
FEE CATEGORY: $10 B(2)$
USE (S)

USE

MAINTENANCE

PROCUREMENT

REPAIR

EXPIRATION DATE : $09 / 30 / 2000$
ORIG. ISSUE DATE : $06 / 20 / 1985$

USE(S)

ORIG. ISSUE DATE: 06/20/1985

APPROVAL TYPE: NORMAL AND SPECIAL FORM

REGULATION: APPENDIX B TO 10 CFR PART 50
FEE CATEGORY: $10 B(2)$

PROCUREMENT MAINTENANCE MAINTENAND
REPAIR USE 
DOCKETH : 07100542

REVISIONH : 2

USER NAME: MATTINGLY TESTING SERVICES, INC. GREAT FALLS
ATTN: MR. MARK M. MATTINGLY
MT 59403

COMMENTS:
EXPIRATION DATE: $06 / 30 / 2000$

APPROVAL TYPE: SPECIAL FORM REGULATION: SUBPART H TO 10 CFR PART 71
FEE CATEGORY: $1 O B(2)$
USE(S)
PROCUREMENT
MAINTENANGE

MAINTENA

USE

DOCKET\# : 07100545

REVISION\#: 5

USER NAME: TN TECHNOLOGIES, INC.

P. O. BOX 800

ROUND ROCK

ATTN: MS. DORIS C. BRYAN

COMMENTS:

DOCKETH : 07100551

REVISION\# : 1

USER NAME: SIEMENS MEDICAL SYSTEMS, INC. 186 WOOD AVENUE, SOUTH

ISELIN ATT : ALFRED F. HORNDASCH
EXPIRATION DATE: $04 / 30 / 1998$
ORIG. ISSUE DATE: $04 / 12 / 1988$

APPROVAL TYPE: NORMAL AND SPECIAL FORM REGULATION: SUBPART H TO 10 CFR PART 71
FEE CATEGORY: $10 B(2)$
$\operatorname{USE}(S)$

USE

MAINTENANCE PROCUREMENT REPAIR

COMMENTS:

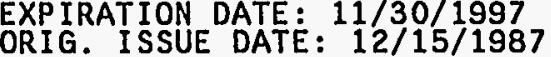

APPROVAL TYPE: NORMAL AND SPECIAL FORM

REGULATION: SUBPART H TO 10 CFR PART 71

FEE CATEGORY: $10 B(2)$
USE(S)

PROCUREMENT MAINTENANCE

REPAIR
USE 
REVISION\#: 3 USER NAME: HIGH MOUNTAIN INSP. SERVICES, INC.
CASPER
ATTN: MR. C.R. CLICK CLINE
WY 82601

COMMENTS: REINSTATED ON JANUARY 29, 1996.

\section{REVISIONH : 2}

USER NAME: VA. POLY INSTITUTE AND STATE UNIVERSITY RADIATION SAFETY OFFICE

BLACKSBURG
ATTN: MR. DOUGLAS C. SMILEY 24061

COMMENTS :
EXPIRATION DATE: $01 / 31 / 2001$
ORIG. I SSUE DATE: $11 / 20 / 1985$

APPROVAL TYPE: SPECIAL FORM

REGULATION: SUBPART H TO 10 CFR PART 71 FEE CATEGORY: $10 B(2)$
USE(S)

PROCUREMENT

MAINTENANCE

REPAIR

MODIFICATION

USE
EXPIRATION DATE: $10 / 31 / 1996$

ORIG. ISSUE DATE:

APPROVAL TYPE: SPECIAL FORM

REGULATION: SUBPART H TO 10 CFR PART 71 FEE CATEGORY: EDUC
DOCKETH : 07100555

USER NAME: ALLEGHENY LABORATORIES

RD \#2 BOX 4-A

KENNERDELL

ATTN: MR. CHARLES MCCLELLAND

\section{REVISION\#:}

EXPIRATION DATE: 04/30/2001

ORIG. ISSUE DATE: $12 / 03 / 1985$

APPROVAL TYPE: SPECIAL FORM

REGULATION: SUBPART H TO 10 CFR PART 71 16374 FEE CATEGORY: IOB(2)
USE (S)

USE

PROCUREMENT 
DOCKET\# : 07100558

REVISION\# : 2

USER NAME: CRAMER \& LINDELL ENGINEERS, NDT LABORATORY 277 WEST MAIN STREET NIANTIC ATTN: MR. D. ORLOSKY

CT 06357

COMMENTS :

\section{REVISION\#: 2}

USER NAME: NON-DESTRUCTIVE TESTING CORPORATION P.O. BOX 127

\section{MANVILLE NJ 08835 \\ ATTN: MR. ANTHONY C. LEWIS}

EXPIRATION DATE : $01 / 31 / 2001$
ORIG. ISSUE DATE: $12 / 03 / 1986$

APPROVAL TYPE: SPECIAL FORM

REGULATION: SUBPART H TO 10 CFR PART 71
$\operatorname{USE}(S)$

PROCUREMENT

MAINTENANCE

REPAIR

USE

COMMENTS :

EXPIRATION DATE : $01 / 31 / 2001$
ORIG. ISSUE DATE: $01 / 09 / 1986$

APPROVAL TYPE: SPECIAL FORM

REGULATION: SUBPART H TO 10 CFR PART 71

FEE CATEGORY: $10 B(2)$

\section{USE (S)}

PROCUREMENT MAINTENANCE REPAIR
DOCKET\# : 07100563

REVISION\#: 2

USER NAME: EDWARDS PIPELINE TESTING, INC.

TULSA MS. ANITA B. LAKE
OK 74147
EXPIRAT ION DATE: $01 / 31 / 2001$

ORIG. ISSUE DATE: $01 / 17 / 1986$

APPROVAL TYPE: SPECIAL FORM

REGULATION: SUBPART H TO 10 CFR PART 71

REGULATION: SUBPART
FEE CATEGORY: $10 B(2)$
$\operatorname{USE}(S)$

PROCUREMENT MAINTENANCE REPAIR

\section{COMMENTS :}


DOCKETH : 07100566

REVISION\#: 3

USER NAME: ENTERGY OPERATIONS, INC., RIVER BEND STATION P.O. BOX 220 5485 U. STGHWAY 61 ST. FRANCI I VILLE ATTN: MR. DAVID LORFING

LA 70775

COMMENTS:

\section{REVISIONH : 3}

USER NAME: ARIZONA PUBLIC SERVICE COMPANY PALO VERDE NUCLEAR GEN. STA.

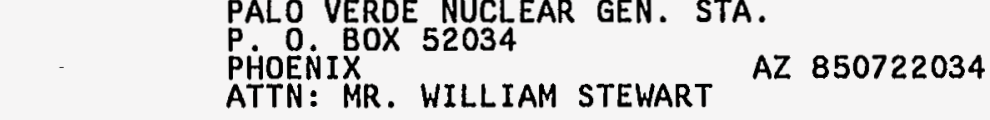
AZ 850722034

EXPIRATION DATE: $02 / 28 / 2001$
ORIG. ISSUE DATE: $01 / 13 / 1986$

APPROVAL TYPE: NORMAL AND SPECIAL FORM

REGULATION: APPENDIX B TO 10 CFR PART 50 FEE CATEGORY: $10 B(2)$

\section{USE(S)}

\section{PROCUREMENT} MAINTENANCE REPAIR

\section{REVISION\# : 3}

USER NAME: PURDUE UNIVERSITY

CIVIL ENGINEERING BUILDING

\section{B173
WEST LAFAYETTE
ATTN: MR. JAMES F. SCHWEITZER 47907}

EXPIRATION DATE: $03 / 31 / 2001$
ORIG. ISSUE DATE: $03 / 19 / 1986$

APPROVAL TYPE: NORMAL AND SPECIAL FORM

REGULATION: APPENDIX B TO 10 CFR PART 50

REGULATION: APPENDIX
FEE CATEGORY: $10 B(2)$

\section{USE(S)}

PROCUREMENT MAINTENANCE USPAIR

COMMENTS:

EXPIRATION DATE:
ORIG. ISSUE DATE:

APPROVAL TYPE: SPECIAL FORM

REGULATION: SUBPART H TO 10 CFR PART 71
FEE CATEGORY: EDUC
USE( $S$ )

MAINTENANCE PROCUREMENT REPAIR 
REVISION\#: 2

USER NAME: UNIVERSITY OF FLORIDA, NUCLEAR REACTOR FACILITY NUCLEAR REACTOR BUILDING

GAINESVILLE ATTN: MR. W.G. VERNETSON FL 32611
EXP IRATION DATE: 10/31/1997

ORIG. ISSUE DATE:

APPROVAL TYPE: SPECIAL FORM

REGULATION: SUBPART H TO 10 CFR PART 71
FEE CATEGORY: EDUC

COMMENTS :

DOCKETH : $07100579 \quad$ REVISION\# : 2

USER NAME: SENIOR ENGINEERING COMPANY 2702 W. 9 TH ST.

JOPLIN MO 64801

ATTN: MR. GARY L. STILES

COMMENTS:

DOCKETH : 07100583

REVISION\#: 2

USER NAME: MINNESOTA VALLEY ENGINEERING INC.

407 SEVENTH ST. N.W.

P. OO BOX 234

ATTN: MR. RICK MINETTE
MN 56071
EXPIRATION DATE: $04 / 30 / 2001$
ORIG. ISSUE DATE: $04 / 29 / 1986$

APPROVAL TYPE: SPECIAL FORM

REGULATION: SUBPART H TO 10 CFR PART 71
FEE CATEGORY: $10 B(2)$

\section{USE(S) \\ MAINTENANCE \\ USE}

COMMENTS:

EXPIRATION DATE:
ORIG. ISSUE DATE:
$02 / 28 / 28 / 1986$

APPROVAL TYPE: SPECIAL FORM

REGULATION: SUBPART H TO 10 CFR PART 71
FEE CATEGORY: $10 B(2)$
USE(S)

PROCUREMENT

MAINTENANCE

REPAIR
USE

PROCUREMENT

MAINTENANCE

REPAIR 
NRC-1265006-006

U.S. NUCLEAR REGULATORY COMMISSION

DOCKETH : 07100585

REVISION\#: 2

USER NAME: MQS INSPECTION INC.

2301 ARTHUR AVE.

ELK GROVE VILLAGE

COMMENTS :

DOCKETH: 07100587

REVISION\# : 4

USER NAME: DEPT. OF AIR FORCE, RADIOISOTOPE COMMITTEE HQ AFOMA/SGPR

STREET

ATTN: LTCOL JOSEPH DONNELLY TX 782355217
EXPIRATION DATE: $03 / 31 / 2001$

ORIG. ISSUE DATE: 03/05/1986

APPROVAL TYPE: SPECIAL FORM

REGULATION: SUBPART H TO 10 CFR PART 71 FEE CATEGORY: $10 B(2)$
USE (S)

PROCUREMENT MAINTENANCE

REPAIR

USE

\section{COMMENTS :}

DOCKETH : 07100592

REVISION\#: 2

USER NAME: BRAUN INTERTEC CORPORATION . O. BOX 39108 6875 WASHINGTON AVENUE SOUTH MINNEAPOLIS
EXPIRATION DATE: 04/30/2001

ORIG. ISSUE DATE: 04/17/1986

APPROVAL TYPE: SPECIAL FORM

REGULATION: SUBPART H TO 10 CFR PART 71 FEE CATEGORY: $1 O B(2)$

\section{$\operatorname{USE}(S)$}

PROCUREMENT MAINTENANCE REPAIR USE

COMMENTS:

EXPIRATION DATE: 05/31/2001

ORIG. ISSSUE DATE : 04/08/1986

APPROVAL TYPE: SPECIAL FORM

REGULATION: SUBPART H TO 10 CFR PART 71 REE CATEGORY: $10 B(2)$
USE(S)

PROCUREMENT MAINTENANCE REPAIR

USE 
EXPIRATION DATE: $05 / 31 / 2001$
ORIG. ISSUE DATE: $04 / 09 / 1986$

APPROVAL TYPE: SPECIAL FORM

TRI STATE INSPECI
115 ISLAND AVE

MCKEES ROCKS PA 15136

COMMENTS:

\section{DOCKET\# : 07100595}

REVISION\#: 1

USER NAME: AKRON INDUSTRIAL SERVICES, INC. 4322 MOGADORE ROAD

KENT . MP. LESTER A. MOORE OH 44240

COMMENTS : REGULATION: SUBPART H TO 10 CFR PART 71
FEE CATEGORY: $10 B(2)$

\section{USE (S)}

PROCUREMENT MAINTENANCE REPAIR

USE

DOCKETH : 07100598

REVISIONH : 3

USE( $S$ )

USER NAME: WOLF CREEK NUCLEAR OPERATING CORP.

P. O. BOX 411

BURLINGTON

ATTN: MR. OTTO L. MAYNARD

KS 66839

COMMENTS:

EXP IRATION DATE: $09 / 30 / 2001$

APPROVAL TYPE: NORMAL AND SPECIAL FORM

EXPIRATION DATE: $08 / 31 / 1997$
ORIG. ISSUE DATE: $05 / 22 / 1986$

APPROVAL TYPE: SPECIAL FORM

REGULATION: SUBPART H TO 10 CFR PART 71
FEE CATEGORY: $10 B(2)$

REGULATION: SUBPART H TO 10 CFR PART 71
FEE CATEGORY: $10 B(2)$

\section{USE \\ MAINTENANCE \\ PROCUREMENT REPAIR}


DOCKET\# : 07100600

REVISION\#: 2

USER NAME: SHAFER VALVE COMPANY 2500 PARK AVE. WEST

MANSFIELD ATTN: MR. CRAIG MCCOMBS

OH 44906
EXPIRATION DATE: $06 / 30 / 2001$
ORIG. I SSUE DATE: $06 / 19 / 1986$

APPROVAL TYPE: SPECIAL FORM

REGULATION: SUBPART H TO 10 CFR PART 71
FEE CATEGORY: $10 B(2)$

\section{USE (S)}

PROCUREMENT MAINTENANCE REPAIR USE

\section{COMMENTS :}

DOCKET\# : 07100603

REVISION\#: 2

USER NAME: JAN X-RAY CO. 8343 MINARD ST.

PARMA MI 49269 ATTN: MR. J. A. NEWLAND

COMMENTS :

\section{REVISION\#: 3}

USER NAME: ENTERGY OPERATIONS, INC. WATERFORD 3 PI ODONAX KILLONA

COMMENTS:

EXPIRATION DATE: $06 / 30 / 2001$
ORIG. ISSUE DATE: $06 / 16 / 1986$

APPROVAL TYPE : SPECIAL FORM

REGULATION: SUBPART H TO 10 CFR PART 71
USE (S)

PROCUREMENT MAINTENANCE REPAIR USE
ORIG. ISSUE DATE: 06/30/1986

APPROVAL TYPE: NORMAL AND SPECIAL FORM

REGULATION: APPENDIX B TO 10 CFR PART 50 FEE CATEGORY: 1OB(2)
USE(S)

PROCUREMENT MAINTENANCE REPAIR USE 


\section{REVISION\# : 1}

USER NAME: SCIENTIFIC MEASUREMENT SYS. IN 2209 DONLEY DRIVE

AUSTIN

ATTN: MR. G. A. MEYER
TX 78758
EXP IRATION DATE: 09/30/1996

ORIG. ISSUE DATE: 09/17/1986

APPROVAL TYPE: SPECIAL FORM

REGULATION: SUBPART H TO 10 CFR PART 71

FEE CATEGORY: $10 B(2)$

COMMENTS :

\section{DOCKET\# : 07100611 \\ REVISION\#: 4}

USER NAME: LIBERTY TECHNICAL SERVICES 8550 DORCHESTER ROAD

NORTH CHARIESTON

ATTN: MR. GERALD WADE

SC 294207301

COMMENTS :
REVISION\#: 1

USER NAME: SPEC CONSULTANTS, INC.

510 PLEASANT VALLEY RD.

TRAFFORD
ATTN: MR. LOUIS C. HASS
DOCKETH : 07100612
PA 15085
EXPIRATION DATE: $09 / 30 / 2001$
ORIG. ISSUE DATE: $09 / 17 / 1986$

APPROVAL TYPE: SPECIAL FORM

REGULATION: SUBPART H TO 10 CFR PART 71

\section{USE (S)}

PROCUREMENT MAINTENANCE REPIR

USE
EXPIRATION DATE: $10 / 31 / 1996$
ORIG. ISSUE DATE: $10 / 02 / 1986$

APPROVAL TYPE: SPECIAL FORM

REGULATION: SUBPART H TO 10 CFR PART 71
FEE CATEGORY: $10 B(2)$
USE (S)

PROCUREMENT MAINTENANC

REPAIR
USE

COMMENTS: 
REVISION\# : 1

USER NAME: DEPARTMENT OF THE ARMY ARMY TEST, MEASUREMENT \& DIAGNOSTIC EQUIP. ACTIVITY REDSTONE ARSENAL KUYKENDALL AL 35898

COMMENTS :

DOCKETH : 07100615

REVISION\# : 1

USER NAME: ALONSO \& CARUS IRON WORKS P. 0. BOX 566. ROAD 869 KM 0. 09, BO PÁLMAS CATANO ATTN: MR. JOSE L. CINTRON
EXP IRATION DATE: $12 / 31 / 1996$

APPROVAL TYPE: SPECIAL FORM

REGULATION: SUBPART H TO 10 CFR PART 71
FEE CATEGORY: $10 B(2)$
USE (S)

PROCUREMENT MAINTENANCE REPAIR USE
ORIG. ISSUE DATE: $11 / 30 / 1996$

APPROVAL TYPE: SPECIAL FORM

REGULATION: SUBPART H TO 10 CFR PART 71 FEE CATEGORY: $10 B(2)$

\section{USE(S)}

PROCUREMENT MAINTENANCE REPAIR USE

\section{COMMENTS :}

DOCKET\# : 07100624

REVISION\#: 2

USER NAME: ILLINOIS POWER COMPANY CLINTON PWR STA., P.O. BOX 678

CLINTTON

IL. 617270678
EXPIRATION DATE: 08/31/2001

APPROVAL TYPE: NORMAL AND SPECIAL FORM

REGULATION: APPENDIX B TO 10 CFR PART 50
USE(S)

PROCUREMENT MAINTENANCE REPAI

COMMENTS : 
DOCKET\# : 07100625

REVISION\# : 1

USER NAME: COPES-VULCAN, INC.

P. O. BOX 577

MARTIN AND RICE AVE.

ATTN: MR. F. R. PORTER

PA 16423

COMMENTS:

DOCKET\# : 07100627

REVISION\#: 1

USER NAME: CONSOLIDATED NDE. INC.

6 WOODBRIDGE AVENUE.

P. O. BOX 593

WOODBRIDG

ATTN: MR. BRUCE R. BALLARD

NJ 07095

COMMENTS :

DOCKET\# : 07100628

REVISION\# : 1

USER NAME: NOVA DATA TESTING LABS

204 NORTH MAIN STREET, \#201

HOPEWELLL
ATTN: MR. JOHN TAYLOR VA 23860

204 NORTH MAIN STREET, $\# 201$
HOPEWELL. JOHN TAYLOR VA 23860
ATTN: MR. JOR

EXP IRATION DATE : $02 / 28 / 1997$
ORIG. ISSUE DATE : $02 / 11 / 1987$

APPROVAL TYPE: SPECIAL FORM

REGULATION: SUBPART H TO 10 CFR PART 71
FEE CATEGORY: $10 B(2)$
USE (S)

PROCUREMENT

MAINTENANCE

REPAIR

USE
EXPIRATION DATE: $03 / 31 / 1997$

APPROVAL TYPE: SPECIAL FORM

REGULATION: SUBPART H TO 10 CFR PART 71

FEE CATEGORY: $1 O B(2)$

\section{$\operatorname{USE}(S)$}

USE

MAI NTENANCE PROCUREMENT REPAIR

\section{COMMENTS :}

EXPIRATION DATE: $03 / 31 / 1997$

APPROVAL TYPE: SPECIAL FORM

REGULATION: SUBPART H TO 10 CFR PART 71

FEE CATEGORY: $10 B(2)$
USE (S)

PROCUREMENT MAINTENANCE REPAIR 
REVISION\# : 1

USER NAME: SCIENTIFIC TECHNICAL, INC. $804 \mathrm{~F}$ INDUSTRIAL AVE.

CHESAPEAKE ATTN: MR. BOBBY L. DENNIS

VA 23324
EXP IRATION DATE: $03 / 31 / 1997$
ORIG. ISSUE DATE: $03 / 23 / 1987$

APPROVAL TYPE: SPECIAL FORM

REGULATION: SUBPART H TO 10 CFR PART 71
USE (S)

PROCUREMENT MAINTENANCE

REPAIR

USE

\section{COMMENTS:}

DOCKET\#：07100630

REVISION\# : 1

USER NAME: PROGRESS SERVICES, INC. 4170 COLUMB IA ROAD

MEDINA

ATTN: MS. M. A. BECKER

$\mathrm{OH} 44256$

COMMENTS:

DOCKETH : 07100632

REVISION\#: 2

USER NAME: QUALITY ENERGY SERVICES AND TESTS CORP. P. 0 . BOX 9501 SERVICES AND
4141 S. GALVESTON STREET TULSA GA J. C. WILSON

OK 74157
EXPIRATION DATE: 04/30/1997

APPROVAL TYPE: SPECIAL FORM

REGULATION: SUBPART H TO 10 CFR PART 71 FEE CATEGORY: 1OB(2)

\section{USE(S)}

PROCUREMENT MAINTENANCE REPAIR USE

\section{COMMENTS:}

EXPIRATION DATE: 07/31/1997

APPROVAL TYPE: SPECIAL FORM

REGULATION: SUBPART H TO 10 CFR PART 71 FEE CATEGORY: $10 B(2)$
USE (S)

PROCUREMENT MAINTENANCE REPAIR 
EXPIRATION DATE: 02/28/1998

ORIG. ISSUE DATE: $02 / 16 / 1988$

APPROVAL TYPE: SPECIAL FORM

H \& G INSPECTION COMPANY, INC.
P. O. BOX 721856

HOUSTON

ATTN: MR. H. W. GIBSON

TX 77272

REGULATION: SUBPART H TO 10 CFR PART 71
FEE CATEGORY: $10 B(2)$
USE(S)

PROCUREMENT

MAINTENANCE

REPAIR

USE

COMMENTS:

\section{DOCKETH : 07100637 \\ REVISION\#: 3 \\ USER NAME: ANALYTIC STRESS RELIEVING, INC. WESTERN STRESS DIVISION 7523 WHITEPINE ROAD RICHMOND. RAYMOND MARTIN \\ VA 23237}

COMMENTS:
EXPIRATION DATE: $11 / 30 / 1997$
ORIG. ISSUE DATE: $11 / 05 / 1987$

APPROVAL TYPE: SPECIAL FORM

REGULATION: SUBPART H TO 10 CFR PART 71

\section{USE ( $S$}

PROCUREMENT MAINTENANCE REPAIR
DOCKETH : 07100638

REVISION\#: 2

USER NAME: WESTINGHOUSE ELECTRIC CORP., NUCLEAR MFG. DIV. BOX 355

PITTSBURGH

ATTN: MR. A. J. NARDI
PA 152300355
EXPIRATION DATE: $10 / 31 / 1998$
ORIG. ISSUE DATE: $11 / 05 / 1987$

APPROVAL TYPE: NORMAL AND SPECIAL FORM

REGULATION: SUBPART H TO 10 CFR PART 71
FEE CATEGORY: $10 B(2)$

COMMENTS : 
DOCKETH : 07100639

REVISION\# : 1

USER NAME: WOS TESTING INC.

E14370 CURLER ROAD

FALL CREEK

WI 54742

COMMENTS :

DOCKETH : 07100640

REVISION\#: 1

USER NAME: PSI ENERGY, INC.

1000 EAST MAIN STREET

PLAINFIELD
ATTN: MR. GENE DAVIS

IN 46168

COMMENTS:

DOCKETH : 07100641

REVISION\#: 4

USER NAME: SGS INDUSTRIAL SERVICES

2506 DAVIS STREET

SAN LEANDRO CA 94577

COMMENTS :

ATTN: MR. TOM W. CUTHBERTSONA 94577

EXPIRATION DATE: 01/31/1998

ORIG. ISSUE DATE: $02 / 16 / 198$

APPROVAL TYPE: SPECIAL FORM

REGULATION: SUBPART H TO 10 CFR PART 71

FEE CATEGORY: 1OB(2)

\section{USE(S)}

PROCUREMENT

MAINTENANCE

REPAIR

USE

EXPIRATION DATE: $10 / 31 / 1997$

ORIG. ISSUE DATE: $11 / 05 / 1987$

USE(S)

APPROVAL TYPE: SPECIAL FORM

PROCUREMENT

PROCUREMENT

REPAIR

USE

REGULATION: SUBPART H TO 10 CFR PART 71
FEE CATEGORY: $10 B(2)$

EXPIRATION DATE: 04/30/1998

ORIG. ISSUE DATE: O4/12/1988

APPROVAL TYPE: SPECIAL FORM

REGULATION: SUBPART H TO 10 CFR PART 71

FEE CATEGORY: $10 B(2)$

\begin{tabular}{l} 
USE (S) \\
\hline USE \\
MAINTENANCE \\
PROCUREMENT \\
REPAIR
\end{tabular}


DOCKETH: 07100642

REVISIONH: 6

EXPIRATION DATE: $11 / 30 / 1997$
ORIG. ISSUE DATE: $02 / 16 / 1988$

APPROVAL TYPE: NORMAL AND SPECIAL FORM

USER NAME: CIS-US, INC. 10 DEANGELOO DRIVE

BEDFORD MA 01730

ATTN: MR. DAVID B. READER

REGULATION: SUBPART H TO 10 CFR PART 71
FEE CATEGORY: $10 B(2)$
USE(S)

PROCUREMENT

MAINTENANCE

REPAIR

USE

COMMENTS:

DOCKET\#： 07100645

REVISION\# : 2

USER NAME: THE LIGHT COMPANY

P. 0. BOX 289

WADSWORTH HOWARD W. BERGENDAHL 77483
EXP IRATION DATE: $03 / 31 / 1998$
ORIG. ISSUE DATE: $02 / 16 / 1988$

APPROVAL TYPE: SPECIAL FORM

REGULATION: SUBPART H TO 10 CFR PART 71
FEE CATEGORY: $10 B(2)$
USE (S)

USE

MAINTENANCE

PROCUREMENT REPAIR

COMMENTS :

\section{DOCKETH: 07100652}

REVISION\#: 2

USER NAME: SAM-SON INSPECTION \& TECHNICAL SERVICES, INC.

P. 0. BOX 80570, STATION C

CANTON
ATTN: MR. S. M. HOPKINS

$\mathrm{OH} 44708$
EXPIRATION DATE: $05 / 31 / 1998$
ORIG. ISSUE DATE:
$05 / 24 / 1988$

APPROVAL TYPE: SPECIAL FORM

REGULATION: SUBPART H TO 10 CFR PART 71

FEE CATEGORY: $10 B(2)$
$\operatorname{USE}(S)$

USE

MAINTENANCE PROCUREMENT REPAIR

COMMENTS: 
NRC-1265006-006

U.S. NUCLEAR REGULATORY COMMISSION

QUALITY ASSURANCE PROGRAMS REPORT

$10 / 01 / 1996$

PAGE

DOCKETH : 07100656

REVISIONH: 1

USER NAME: PROFESSIONAL WELDING ASSOCIATES, INC. E3349 CITY HIGHWAY G

KEWAUNEE GARY MARQUARDT WI 54216

COMMENTS:

DOCKETH : 07100659

REVISION\#: 2

USER NAME: TRI STATE ASSOCIATES, INC. P. 0. BOX 173

HARTWOOD VA 22471

ATTN: MS. JOANN DUNN

COMMENTS:

DOCKETH : 07100660

REVISION\#: 1

USER NAME: ATOMIC INSEPCTION LABS.

5620 MODESTO N.E.

ALBUQUERQUE

ATTN: MR. KLAY L. ROBERTS

NM 87113
EXPIRATION DATE: $11 / 30 / 1998$
ORIG. I SSUE DATE: $08 / 02 / 1988$

APPROVAL TYPE: SPECIAL FORM

REGULATION: SUBPART H TO 10 CFR PART 71

FEE CATEGORY: $10 B(2)$
USE(S)

USE

PROCUREMENT

REPAIR

COMMENTS:

EXPIRATION DATE: 09/30/1998

ORIG. ISSUE DATE: 09/20/1988

APPROVAL TYPE: SPECIAL FORM

REGULATION: SUBPART H TO 10 CFR PART 71

FEE CATEGORY: IOB(2)
USE(S)

USE

MAINTENANCE PROCUREMENT REPAIR
EXPIRATION DATE:
ORIG. ISSUE DATE: $11 / 30 / 1998$
$10 / 20 / 1988$

APPROVAL TYPE: SPECIAL FORM

REGULATION: SUBPART H TO 10 CFR PART 71

FEE CATEGORY: $10 B(2)$

\begin{tabular}{l} 
USE(S) \\
\hline USE \\
MAINTENANCE \\
PROCUREMENT \\
REPAIR
\end{tabular}


EXP IRATION DATE: $12 / 31 / 1998$

ORIG. ISSUE DATE: $10 / 24 / 1988$

APPROVAL TYPE: SPECIAL FORM

USER NAME: BARNETT INDUSTRIAL X-RAY, INC. STILLWATER

COMMENTS:

DOCKETH : 07100667

REVISION\#: 1

USER NAME: PITT-DES MOINES INC.
3400 GRAND AVENUE

PITTSBURGH ATTN: MR. J. F. STRUNK

PA 15225

COMMENTS:

DOCKETH : 07100669

REVISION\#: 1 USER NAME: COMO TECH INSPECTION SERVICE
27640 WEST 83RD STREET

LENEXA ATTN: MR. R. E. HIERSCHE
REGULATION: SUBPART H TO 10 CFR PART 71 FEE CATEGORY: $1 O B(2)$
USE (S)

USE

MAINTENANCE

REMEN REPAIR

COMMENTS:

EXPIRATION DATE: $03 / 31 / 1999$
ORIG. ISSUE DATE : $02 / 21 / 1989$

APPROVAL TYPE: SPECIAL FORM REGULATION: SUBPART H TO 10 CFR PART 71
FEE CATEGORY: $10 B(2)$

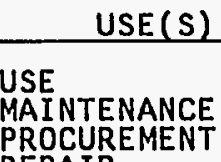

MAINTENANCE REPAIR
EXPIRATION DATE: $02 / 28 / 1999$
ORIG. ISSUE DATE: $04 / 05 / 1989$

APPROVAL TYPE: SPECIAL FORM

REGULATION: SUBPART H TO 10 CFR PART 71

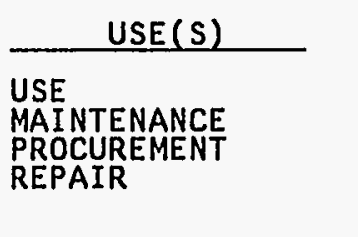
( REPAIR 
DOCKET\# : 07100672

REVISION\# : 1 USER NAME: LUCIUS PITKIN INC.

NEW YORK ATTN: MR. ROBERT F. PLUMSTEY 10013

COMMENTS:
REVISION\#: 1

USER NAME: THIELSCH ENGINEERING ASSOC. 195 FRANCES AVE.

CRANSTON BRUCE H. BURDICK RI 02910

COMMENTS :

DOCKETH : 07100677

REVISION\#: 2

USER NAME: ANVIL CORPORATION

742 SOUTH SPRUCE STREET

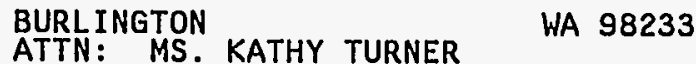

COMMENTS :
EXP IRATION DATE: 03/31/1999

ORIG. ISSUE DATE: O4/05/1989

APPROVAL TYPE: SPECIAL FORM

REGULATION: SUBPART H TO 10 CFR PART 71 FEE CATEGORY: $10 B(2)$
USE(S)

USE

MAINTENANCE

PROCUREMENT

REPAIR
EXPIRATION DATE: 03/31/1999

APPROVAL TYPE: SPECIAL FORM

REGULATION: SUBPART H TO 10 CFR PART 71 FEE CATEGORY: $1 O B(2)$ \begin{tabular}{l} 
USE(S) \\
\hline USE \\
MAINTENANCE \\
PROCUREMENT
\end{tabular} REPAIR
EXPIRATION DATE: 04/30/1997 ORIG. ISSUE DATE: 05/09/1989

APPROVAL TYPE: SPECIAL FORM

REGULATION: SUBPART H TO 10 CFR PART 71 FEE CATEGORY: $10 B(2)$

\begin{tabular}{l} 
USE(S) \\
\hline USE \\
MAINTENANCE \\
PROCUREMENT \\
REPAIR
\end{tabular}


REVISION\# : 2

USER NAME: PACKAGING TECHNOLOGY. INC. 4507-D PACIFIC HIGHWAY EAST

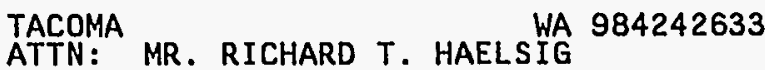

EXPIRATION DATE: 09/30/1999

ORIG. ISSUE DATE: 09/19/1989

APPROVAL TYPE: NORMAL AND SPECIAL FORM

REGULATION: SUBPART H TO 10 CFR PART 71 FEE CATEGORY: 1OB(1)
USE (S)

USE

MAINTENANCE MOD IFICATION REPAIR ASSEMBLY AOR I FABRICATION TESTING

\section{COMMENTS :}

DOCKETH : 07100679

REVISION\# : 1

USER NAME: TESTMASTER INSPECTION COMPANY

PERRYSBURG
ATTN: MR. W. B. CARR OH 43551
EXPIRATION DATE: $07 / 31 / 1999$
ORIG. I SSUE DATE: $07 / 31 / 1989$

APPROVAL TYPE: SPECIAL FORM REGULATION: SUBPART H TO 10 CFR PART 71
FEE CATEGORY: $10 B(2)$

COMMENTS :

DOCKET\#: 07100680

REVISION\#: 2

USER NAME: THE OHIO STATE UNIVERSITY B-042 GRAVES HALL COLUMBUS OH 432101239

ATTN: MR. MICHAEL J. DAVIS
EXPIRATION DATE: 06/30/1998

ORIG. ISSUE DATE:

APPROVAL TYPE: SPECIAL FORM

REGULATION: APPENDIX B TO 10 CFR PART 50 FEE CATEGORY: EDUC

COMMENTS : 
DOCKETH : 07100682

REVISION\#: 1

USER NAME: PACIFIC TECHNICAL INDUSTRIES, INC. $1414127 T H$ PLACE, N.E. NO. 107 BTTLEVUE MR. DOUGLAS L. NALLEY 98005

COMMENTS:

DOCKET\# : 07100683

REVISION\#: 1

USER NAME: TESTING TECHNOLOGIES, INC.

WOODBRIDGE
ATTN: MS. JUDY CASE VA 22191

COMMENTS:

DOCKET\# : 07100685

REVISION\#: 1

USER NAME: SIERRA TESTING, INC,

P. 0. BOX 9858

TULSA MR. ANTHONY L. SPENCER 74157
USE (S)

EXPIRATION DATE: $12 / 31 / 1999$

APPROVAL TYPE: SPECIAL FORM

REGULATION: SUBPART H TO 10 CFR PART 71

FEE CATEGORY: $10 B(2)$
MAINTENANCE PROCUREMENT REPAIR

\section{COMMENTS :}

EXPIRATION DATE: 08/31/1999

APPROVAL TYPE: SPECIAL FORM

REGULATION: SUBPART H TO 10 CFR PART 71 FEE CATEGORY: $10 B(2)$

\section{USE(S)}

USE

MAINTENANCE MOCUREMENT REPAIR 


\section{REVISION\# : 1}

USER NAME: INSPECTION MANAGEMENT CORPORATION 5626 E. 186TH STREET $S$.

BIXBY ATTN : MR. D. L. MYERS

OK 74008
EXP IRATION DATE: $11 / 30 / 1999$

ORIG. ISSUE DATE: $01 / 31 / 1990$

APPROVAL TYPE: SPECIAL FORM

REGULATION: SUBPART H TO 10 CFR PART 71 FEE CATEGORY: $10 B(2)$

COMMENTS:

DOCKET\# : 07100691

REVISION\# : 1

USER NAME: TEI ANALYTICAL SERVICES, INC.

P. O. BOX 534

35 WEST POINT ROAD WASHINGTON ATTN: MR. G. E. WEISS, RSO PA 15301

COMMENTS:
REVISION\#: 1

USER NAME: T\&K INSPECTION

WILLISTON ATTN: MR. KENNETH S. KAIN ND 588021591

DOCKETH: 07100692
EXPIRAT ION DATE: $01 / 31 / 2000$
ORIG. ISSUE DATE: $02 / 02 / 1990$

APPROVAL TYPE: SPECIAL FORM

REGULATION: SUBPART H TO 10 CFR PART 71
$\operatorname{USE}(S)$

USE

MAINTENANCE ROCUREMENT REPAIR
EXPIRATION DATE: $09 / 30 / 2000$

APPROVAL TYPE: SPECIAL FORM

REGULATION: SUBPART H TO 10 CFR PART 71
FEE CATEGORY: $10 B(2)$
USE (S)

USE

MAINTENANCE

PROCUREMEN

REPAIR

COMMENTS: 
EXPIRATION DATE: $02 / 28 / 2000$

APPROVAL TYPE: SPECIAL FORM CENTERIOR SERVI
G670 BETA DRIVE

MAYFIELD VILLAGE

ATTN: MR. ROLLAND J. STANDISH

REGULATION: SUBPART H TO 10 CFR PART 71 FEE CATEGORY: $10 B(2)$
USE(S)
USE
MAINTENANEE
PROCUREMENT REPAIR

COMMENTS:

DOCKET\# : 07100694

USER NAME: MID AMERICAN INSPECTION SERVICES, INC. 1206 EFFIE ROAD, P.O. BOX 1427

GAYLORD MR. TERRY L. WILKINS

MI 49735

COMMENTS :
EXP IRATION DATE: $03 / 31 / 2000$
ORIG. ISSUE DATE: $03 / 28 / 1990$

APPROVAL TYPE: SPECIAL FORM

REGULATION: SUBPART H TO 10 CFR PART 71 FEE CATEGORY: $1 O B(2)$
USE (S)

USE

MAINTENANCE PROCUREMENT REPAIR
USER NAME: DEPARTMENT OF THE NAVY, CHIEF OF NAVAL OPERATIONS AAPTAIN J. W. MALINOSKI (455) 22202
REVISION\#: 3 CRYSTAL PLAZA 5 , RM 678 2211 JEFFERSON DAVIS HWY

DOCKET\# : 07100697
EXPIRATION DATE: $04 / 30 / 2000$ ORIG. ISSUE DATE: 05/03/1990

APPROVAL TYPE: NORMAL AND, SPECIAL FORM

REGULATION: SUBPART H TO 10 CFR PART 71 FEE CATEGORY: $10 B(2)$

\begin{tabular}{l} 
USE(S) \\
\hline DESIGN \\
USE \\
MAINTENANCE \\
PROCUREMENT \\
REPAIR
\end{tabular}

COMMENTS : 
EXPIRATION DATE: $04 / 30 / 2000$
ORIG. ISSUE DATE: $06 / 06 / 1990$

APPROVAL TYPE: SPECIAL FORM

QUALITY INSPECTION SERVICES, INC

BUFFALO ATTN: MR. KENNETH W. SCHABERT 142150732

COMMENTS:

\section{DOCKET\#: 07100701}

REVISION\#: 1

USER NAME: NDT SPECIALISTS INC.

1739 SOUTH CARFERRY DRIVE

MILWAUKEE

COMMENTS:

DOCKET\#: 07100703

REVISION\#: 2

USER NAME: NORDION INTERNATIONAL INC.

P. 0. BOX 13500

KANATA, CANADA ATTN: MR. P. A. GRAY

$K 2 K 1 \times 8$

EXP IRATION DATE: $07 / 31 / 2000$
REGULATION: SUBPART H TO 10 CFR PART 71
FEE CATEGORY: $10 B(2)$
USE(S)

USE

MAINTENANCE

PROCUREMENT

REPAIR
EXPIRATION DATE: $07 / 31 / 2000$
ORIG. ISSUE DATE : $06 / 20 / 1990$

APPROVAL TYPE: SPECIAL FORM

REGULATION: SUBPART H TO 10 CFR PART 71
FEE CATEGORY: $10 B(2)$
USE(S)

USE

MAINTENANCE PROCUREMENT REPAIR
APPROVAL TYPE: NORMAL AND SPECIAL FORM

REGULATION: SUBPART H TO 10 CFR PART 71

FEE CATEGORY: $10 B(1)$
USE(S)

ASSEMBLY MOD IFICATION FABRICATION TESTING DESIGN USE MAINTENANCE PROCUREMENT REPAIR

COMMENTS : 
NRC-1265006-006

U.S. NUCLEAR REGULATORY COMMISSION

QUALITY ASSURANCE PROGRAMS REPORT 10/01/1996

DOCKETH : 07100705

REVISION\# : 1

USER NAME: APPLIED TECHNICAL SERVICES, INC.

1190 ATLANTA INDUSTRIAL DRIV

MARIETTA GENE MOCK GA 30066

COMMENTS:

DOCKETH : 07100706

\section{REVISION\#: 1}

USER NAME: S. K. MCBRYDE, INC.

SUMMERF IELD

SUMMERF IELD

COMMENTS:
DOCKETH : 07100707

REVISIONH : 1

USER NAME: CARONDELET CORPORATION

P. 0. BOX 769

8600 COMMERCIAL BOULEVARD

PEVELY MR. RICHARD HUBER MO 630700769
EXPIRATION DATE: 04/30/2000

APPROVAL TYPE: SPECIAL FORM

REGULATION: SUBPART H TO 10 CFR PART 71

FEE CATEGORY: $10 B(2)$
USE (S)

USE

MAINTENANCE

PROCUREMENT

PROCUREME
REPAIR

COMMENTS:

EXPIRATION DATE: $08 / 31 / 2000$

ORIG. ISSUE DATE : O8/29/1990

APPROVAL TYPE: SPECIAL FORM

REGULATION: SUBPART H TO 10 CFR PART 71

FEE CATEGORY: $10 B(2)$ USE (S)
USE
MAINTENANCE
PROCUREMENT
REPAIR
EXPIRATION DATE: $12 / 31 / 1999$
ORIG. ISSUE DATE : $08 / 29 / 1990$

APPROVAL TYPE: SPECIAL FORM

REGULATION: SUBPART H TO 10 CFR PART 71 FEE CATEGORY: $1 O B(2)$
USE (S)

USE

MAINTENANCE PROCUREMENT REPAIR 
EXP IRATION DATE: $03 / 31 / 2001$

ORIG. ISSUE DATE: 10/29/1990

USER NAME: WESTINGHOUSE ELECTRIC CORP.. ENERGY SYS. BUS. UNIT PITTSBURGH ATTN: MR. A. J. NARDI PA 15230

APPROVAL TYPE: NORMAL AND SPECIAL FORM

REGULATION: SUBPART H TO 10 CFR PART 71 FEE CATEGORY: $10 B(1)$

COMMENTS:

DOCKETH : 07100711

REVISION\#: 2

USER NAME: NEN LIFE SCIENCES COMPANY 549 ALBANY STREET

BOSTON MR. LEONARD R. SMITH ${ }^{\text {MA } 02118}$

COMMENTS:
REVISIONH: 1

USER NAME: MAXIM TECHNOLOGIES, INC./EMPIRE. SOILS INVEST. P. 0. BOX 2199. 5 KNABANER ROAD

BALLSTON SPA
EXPIRATION DATE: $06 / 30 / 2001$
ORIG. ISSUE DATE: $02 / 12 / 1991$

APPROVAL TYPE: NORMAL AND SPECIAL FORM

REGULATION: SUBPART H TO 10 CFR PART 71
USE (S)

USE

PROOCUREMENT

FABRICATION

DESIGN

COMMENTS:

EXPIRATION DATE: $02 / 28 / 2001$

APPROVAL TYPE: SPECIAL FORM

REGULATION: SUBPART H TO 10 CFR PART 71

$\frac{\text { USE }(S)}{\text { USE }}$

$\operatorname{USE}(S)$

USE MAINTENANCE REPAIR 
DOCKET\# : 07100718

REVISION\#: 1

USER NAME: ARCTIC SLOPE INSPECTION SERVICES, INC. 301 ARCTIC SLOPE AVE.

ANCHORAGE
ATTN: MAME SAMES SCHAEFER, RSO 99518

COMMENTS :

DOCKET\#: 07100721

REVISION\#: 2

USER NAME: QSL INSPECTION INC.

HUNTINGDON VALLEY
ATTN: MR. MICHAEL J. LANGE

$10 / 01 / 1996$
EXP IRATION DATE: 07/31/2001

APPROVAL TYPE: SPECIAL FORM

REGULATION: SUBPART H TO 10 CFR PART 71 FEE CATEGORY: $1 O B(2)$
USE (S)

USE PROCUREMENT REPAIR
EXPIRATION DATE: $06 / 30 / 2001$

ORIG. ISSUE DATE: $06 / 25 / 1991$

APPROVAL TYPE: SPECIAL FORM

REGULATION: SUBPART H TO 10 CFR PART 71 FEE CATEGORY: 1OB(2)

COMMENTS :

\section{DOCKET\# : 07100724}

REVISION\#: 0

USER NAME: VALLEY, INSPECTION SERVICE, INC. P. O. BOX 3245

PALMER
ATTN: MR. ROBERT W. KRATZER 18042
EXPIRATION DATE: $10 / 31 / 1996$ ORIG. ISSUE DATE: 11/01/1991

APPROVAL TYPE: SPECIAL FORM

REGULATION: SUBPART H TO 10 CFR PART 71 FEE CATEGORY: $10 B(2)$
USE(S)

USE

MAINTENANCE PROCUREMENT REPAIR

COMMENTS: 
EXPIRATION DATE: $09 / 30 / 2001$
ORIG. ISSUE DATE: $09 / 18 / 1991$

APPROVAL TYPE: NORMAL AND SPECIAL FORM

USER NAME: GEORGIA POWER COMPANY, YOGTLE PROJECT 40 INVERNESS CENTER PÁRKWAY. P. BOX 1295 BIRMINGHAM ATTN: MR. C. K. MCCOY

AL 35201

COMMENTS :
REGULATION: APPENDIX B TO 10 CFR PART 50
FEE CATEGORY: 1 OB(2)
REVISION\# : 0

USER NAME: CONTINENTAL AIRLINES 8250 EAST SMITH ROAD

DENVER MR. RICHARD L. ROHRIG 80207

COMMENTS :
USE(S)

USE

MAINTENANCE PROCUREMENT REPAIR

\section{DOCKETH : 07100728}

REVISION\#: 0

USER NAME: ACCU-TECH EVALUATION SERVICES, INC. 1410 PINEWOOD STREE

RAHWAY
ATTN: MR. JOSEPH J. FERENC

COMMENTS :
EXPIRATION DATE: $11 / 30 / 1996$
ORIG. ISSUE DATE: $11 / 18 / 1991$

APPROVAL TYPE: SPECIAL FORM

REGULATION: SUBPART H TO 10 CFR PART 71

FEE CATEGORY: $10 B(2)$
USE (S)

USE

MAINTENANCE PROCUREMENT REPAIR
EXP IRATION DATE: $10 / 31 / 1996$ ORIG. I SSUE DATE: $10 / 23 / 1991$ APPROVAL TYPE: SPECIAL FORM

REGULATION: SUBPART H TO 10 CFR PART 71 FEE CATEGORY: $10 B(2)$
$\operatorname{USE}(S)$

USE MAINTENANCE PROCUREMENT REPAIR 
DOCKETH : 07100729

REVISION\#: 0

USER NAME: PAUL SINN TESTING SERVICES BOX 185

MARISSA:

EXP IRATION DATE: $10 / 31 / 1996$ APPROVAL TYPE: SPECIAL FORM REGULATION: SUBPART H TO 10 CFR PART 71
FEE CATEGORY: $10 B(2)$
USE (S)

USE

MAINTENANCE PROCUREMENT REPAIR

COMMENTS:

DOCKETH : 07100731

\section{REVISION\#: 1}

USER NAME: QUALITY INSPECTION \& TESTING, INC. 3111 DAVIS ROAD

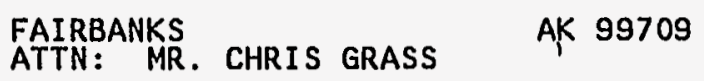

COMMENTS:

\section{REVISION\#: 0}

USER NAME: TAMPELLA KEELER

P.O. BOX 3308, 2600 PEACH ROAD

PA 177010308

\section{WILLIAMSPORT}

EXP IRATION DATE: $12 / 31 / 1996$

APPROVAL TYPE: SPECIAL FORM

REGULATION: SUBPART H TO 10 CFR PART 71 FEE CATEGORY: $10 B(-2)$
USE (S)

USE

MAINTENANCE PROCUREMENT PROCUREM
REPAIR ATTN: MR. R. R. DELKER

\section{COMMENTS:}

EXPIRATION DATE: $12 / 31 / 1996$
ORIG. ISSUE DATE: $12 / 20 / 1991$

APPROVAL TYPE: SPECIAL FORM REGULATION: SUBPART H TO 10 CFR PART 71
FEE CATEGORY: $10 B(2)$

\begin{tabular}{l} 
USE(S) \\
\hline USE \\
MAINTENANCE \\
PROCUREMENT \\
REPAIR
\end{tabular}


EXPIRATION DATE: $02 / 28 / 2002$ ORIG. ISSUE DATE: 02/27/1992

APPROVAL TYPE: SPECIAL FORM

P. O. BOX 2342

$725 B$ E. MOUNTAIN

KERNERSVILLE

ATTN: MR. L. L. SPANGLER NC 27285

COMMENTS:

DOCKETH : 07100734

REVISION\#: 0

USER NAME: METALS EVALUATION AND TESTING, INC. 35 HEGENBERGER PLACE

OAKLAND ATTN: BR. BROOK MASSEY

CA 94621

COMMENTS:

DOCKETH : 07100736

REVISION\#: 0

USER NAME: MAGNA CHEK

\footnotetext{
2125 RIGGS STREET

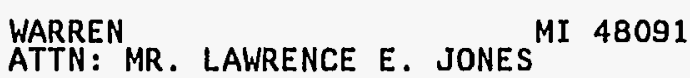

WARREN
}

REGULATION: SUBPART H TO 10 CFR PART 71
FEE CATEGORY: $10 B(2)$
USE (S)

USE

MAINTENANCE

PROCUREMEN

REPAIR

COMMENTS :

EXPIRATION DATE: $05 / 31 / 1997$

APPROVAL TYPE: SPECIAL FORM

REGULATION: SUBPART H TO 10 CFR PART 71

\begin{tabular}{l} 
USE(S) \\
\hline USE \\
MAINTENANCE \\
PROCUREMENT \\
REPAIR
\end{tabular}

EXPIRATION DATE: $06 / 30 / 1997$
ORIG. ISSUE DATE: $07 / 10 / 1992$

APPROVAL TYPE: SPECIAL FORM

REGULATION: SUBPART H TO 10 CFR PART 71
$\operatorname{USE}(S)$

USE

MAINTENANCE

PROCUREMENT REPAIR 
NRC-1265006-006

U.S. NUCLEAR REGULATORY COMMISSION

DOCKETH : 07100737

REVISION\# : 1

USER NAME: AGRA EARTH \& ENVIRONMENTAL, INC. 3232 W. VIRGINIA AVENUE

PHOENIX

COMMENTS :
REVISION\#：0

USER NAME: VERMONT NONDESTRUCTIVE TESTING, INC. 401 PATCHEN ROAD

SOUTH BURLINGTON

ATTN: MR RICHARD F. IRICK VT 05403

COMMENTS :

DOCKET\# : 07100740

\section{REVISION\#: 0}

USER NAME: MIDLAND INSPECTION AND ENGINEERING, INC. 14214 WEST US HIGHWAY 80 EAST

ADESSA ATN: MR. RICHARD C. HAGER IXII 797659406

\section{ODESSA}

EXPIRATION DATE: 07/31/1997

APPROVAL TYPE: SPECIAL FORM

REGULATION: SUBPART H TO 10 CFR PART 71 FEE CATEGORY: $10 B(2)$
USE (S)

USE PROCUREMENT REPAIR
EXP IRATION DATE: 06/30/1997

APPROVAL TYPE: SPECIAL FORM REGULATION: SUBPART H TO 10 CFR PART 71
FEE CATEGORY: $10 B(2)$

\begin{tabular}{l} 
USE(S) \\
\hline USE \\
MAINTENANCE \\
PROCUREMENT \\
REPAIR
\end{tabular}
PROCUREMENT REPAIR

COMMENTS :

EXPIRATION DATE: 07/31/1997 ORIG. ISSUE DATE: 07/30/1992 APPROVAL TYPE: SPECIAL FORM REGULATION: SUBPART H TO 10 CFR PART 71
FEE CATEGORY: $10 B(2)$

\begin{tabular}{l} 
USE(S) \\
\hline USE \\
MAINTENANCE \\
PROCUREMENT \\
REPAIR
\end{tabular}




\section{DOCKET\#： 07100741}

REVISION\#: 0

USER NAME: BIX TESTING LABORATORIES

608 ROLLIINGBROOK DRIVE

BAYTOWN
ATTN: MR. KENNETH R. JOSLYN 77522

COMMENTS :
EXPIRATION DATE: 07/31/1997

APPROVAL TYPE: SPECIAL FORM

REGULATION: SUBPART H TO 10 CFR PART 71

FEE CATEGORY: $1 O B(2)$
USE(S)

USE

MAINTENANCE

PROCUREMENT

REPAIR

$\begin{array}{ll}\text { DOCKET\#: } 07100743 \quad \text { REVISIONH : } 0 \\ \text { USER NAME: } & \text { AMERICAN ASSOCIATED TESTING, INC. } \\ & \text { P.O. BOX } 7054 \\ & \text { LAKE WORTH } \\ & \text { ATTN: }{ }_{\text {MRR. PETER RAMIREZ }}^{\circ} \text { FL } 33466 \\ \text { COMMENTS: } & \end{array}$

EXPIRATION DATE : 09/30/1997

ORIG. ISSUE DATE: 09/09/1992

APPROVAL TYPE: SPECIAL FORM

REGULATION: SUBPART H TO 10 CFR PART 71 FEE CATEGORY: 1OB(2)
USE (

USE

MAINTENANCE

PROCUREMENT

REPAIR

COMMENTS :

\section{DOCKET\# : 07100744}

REVISION\# : 0

USER NAME: X-RAY COMPANY, INC.

810 EAST MAIN

ROBINSON

ATTN: MR. PETER RAMIREZ
IL 62454
EXPIRATION DATE: 09/30/1997

ORIG. I SSUE DATE: 09/09/1992

APPROVAL TYPE: SPECIAL FORM

REGULATION: SUBPART H TO 10 CFR PART 71 FEE CATEGORY: $10 B(2)$

COMMENTS : 
NRC-1265006-006

U.S. NUCLEAR REGULATORY COMMISSION QUALLITY ASSURANCE PROGRAMS REPORT $10 \% 1 / 1996$

EXPIRATION DATE: $09 / 30 / 1997$

ORIG. ISSUE DATE: 10/01/1992

APPROVAL TYPE: SPECIAL FORM

2815 LILAC

PASADENA * NORMAN P. LANIER TX 77503 FEE CATEGORY: $10 B(2)$
USE (S)

USE

MAINTENANCE PROCUREMENT REPAIR

COMMENTS:

REGULATION: SUBPART H TO 10 CFR PART 71

EXP IRATION DATE: 09/30/1997 ORIG. ISSUE DATE: 10/01/1992 APPROVAL TYPE: SPECIAL FORM

RIVEST TESTINE 115 W. 41 STREET TULSA

ATTN: MR. JIM F. KELLY

OK 74107

COMMENTS:

DOCKET\# : 07100750

REVISION\#: 0

USER NAME: BURNLEY TECHNOLOGY, INC.

PLAISTOW

PLAISTOW
REGULATION: SUBPART H TO 10 CFR PART 71 FEE CATEGORY: $1 O B(2)$
$\operatorname{USE}(S)$

USE

MAINTENANCE PROCUREMENT REPAIR
EXPIRATION DATE: 09/30/1997 ORIG. ISSUE DATE: 10/01/1992

APPROVAL TYPE: NORMAL AND SPECIAL FORM

REGULATION: SUBPART H TO 10 CFR PART 71
FEE CATEGORY: $10 B(1)$
USE (S)

USE MAINTENANCE ASSEMBLY MODIFICATION PROCUREMENT FABRICATION REPAIR TESTING

COMMENTS: 


\section{DOCKETH : 07100751}

REVISION\# : 1

USER NAME: S\&ME INC.

$3100^{\prime}$ SPRING FOREST RD.

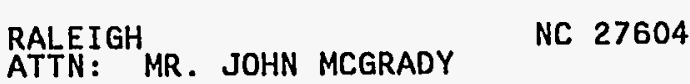

EXPIRATION DATE: $11 / 30 / 1997$

ORIG. ISSUE DATE: 11/09/1992

APPROVAL TYPE: SPECIAL FORM

REGULATION: SUBPART H TO 10 CFR PART 71
FEE CATEGORY: $10 B(2)$

COMMENTS:

DOCKETH : 07100753

REVISION\#: 0

USER NAME: MOBILE-LAB, INC.

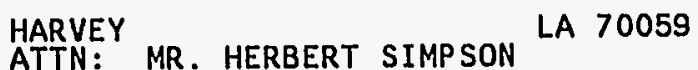

COMMENTS:

DOCKET\#: 07100754

REVISION\#: 0

USER NAME: CODE SERVICES, INC. 26412 OLD HIGHWY 20

MADISON
ATTN: MR. KEVIN E. GREER AL 35758

\section{COMMENTS :}

COMENTS:
EXPIRATION DATE: $01 / 31 / 1998$

APPROVAL TYPE: SPECIAL FORM

REGULATION: SUBPART H TO 10 CFR PART 71
FEE CATEGORY: $10 B(2)$
USE (S)

USE

MAINTENANCE

REPAIR 
REVISION\#: 0

USER NAME: U. S. ARMY COMMUNICATIONS ELECTRONICS COMMAND AMSEL-SF-RE RADIOLOGICAL ENGINEERING BR FORT MONMOUTH ATTN: MR. STEVEN A. HORNE

COMMENTS:

DOCKETH: 07100757

REVISION\#: 0

USER NAME: WELDTEK TESTING LABS., INC. OXFORD ATTN: MR. GARY D. CABLE

AL 36203
EXP IRATION DATE: $12 / 31 / 1997$ ORIG. ISSUE DATE : 01/12/1993

APPROVAL TYPE: SPECIAL FORM

REGULATION: SUBPART H TO 10 CFR PART 71 FEE CATEGORY: $10 \mathrm{~B}(2)$
USE(S)

USE

MAINTENANCE

ROCUREMEN REPAIR

\section{COMMENTS :}

DOCKETH : 07100759

REVISION\#: 0

USER NAME: NATIONWIDE TESTING SERVICES, INC. B11 MORSE AVENUE

SCHAUMBURE ATTN: MR. DAVID H. SIMON
EXPIRATION DATE: $01 / 31 / 1998$
ORIG. I SSUE DATE : $12 / 06 / 1993$

APPROVAL TYPE: SPECIAL FORM

REGULATION: SUBPART H TO 10 CFR PART 71 FEE CATEGORY: $10 B(2)$
USE(S)

USE

MAINTENANCE PROCUREMEN REPAIR

\section{COMMENTS :}

EXPIRATION DATE: 03/31/1998 ORIG. I SSUE DATE: 03/09/1993 APPROVAL TYPE: SPECIAL FORM

REGULATION: SUBPART H TO 10 CFR PART 71 REGULATION:
FEE CATEGORY: $10 B(2)$
$\operatorname{USE}(S)$

USE

MAINTENANCE PROCUREMEN REPAIR 
DOCKETH : 07100761

REVISION\#: 0

USER NAME: KOONEY X-RAY, INC.

COMMENTS:

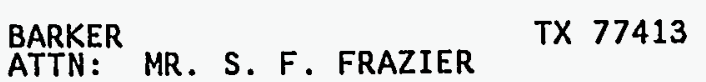

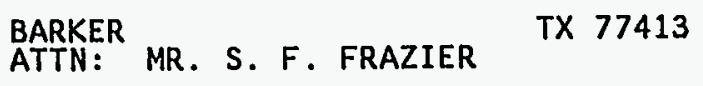

EXPIRATION DATE: $03 / 31 / 1998$

APPROVAL TYPE: SPECIAL FORM

REGULATION: SUBPART H TO 10 CFR PART 71

FEE CATEGORY: $1 O B(2)$
USE(S)

USE

TINTENANCE

PROCUREMENT

REPAIR

\section{DOCKETH: 07100762 \\ REVISIONH: 0}

USER NAME: KC INSPECTION INC.

P. O. BOX 5489

VENTURA
EXPIRATION DATE: 02/28/1998

ORIG. ISSUE DATE : 02/18/1993

APPROVAL TYPE: SPECIAL FORM

REGULATION: SUBPART H TO 10 CFR PART 71

FEE CATEGORY: $1 O B(2)$

COMMENTS:

DOCKET\# : 07100764

REVISION\#: 0

USER NAME: DIAMOND H. TESTING INC.

CHUBBUCK ATTN: PETE J. HANGES ID 83202
EXPIRATION DATE: 04/30/1998

ORIG. ISSUE DATE : 04/16/1993

APPROVAL TYPE: SPECIAL FORM

REGULATION: SUBPART H TO 10 CFR PART 71

FEE CATEGORY: $10 B(2)$
USE (S)

USE

MAINTENANCE

PROCUREMENT

REPAIR

COMMENTS : 
REVISION\#: 0

USER NAME : WESTEX COMPANY
P. O. BOX 5587

OXNARD MR. KEN GRAYBILL CA 93031

COMMENTS :

DOCKETH : 07100768

REVISION\# : 1

EXPIRATION DATE: 09/30/2001

ORIG. ISSUE DATE: 07/08/1993

APPROVAL TYPE: SPECIAL FORM ADESSA MR. ELIC R. BRYMER TX 79763

REGULATION: SUBPART H TO 10 CFR PART 71 FEE CATEGORY: $1 O B(2)$

EXPIRATION DATE: 06/30/1998

ORIG. ISSUE DATE: 06/25/1993

APPROVAL TYPE: SPECIAL FORM

REGULATION: SUBPART H TO 10 CFR PART 71 FEE CATEGORY: $10 B(2)$

\section{USER NAME: DESERT INDUSTRIAL X-RAY, INC.
7408 ANDREWS HIGHWAY}

USE(S)

USE

MAINTENANCE

PROCUREMENT REPAIR COMMENTS:

DOCKETH : 07100760

REVISION\# : 0

USER NAME: GREAT LAKES TESTING, INC. 3171 GROSS STREET

GREEN BAY

ATTN: MR. COREY BUCHER
WI 54304
EXPIRATION DATE: $07 / 31 / 1998$
ORIG. ISSUE DATE: $07 / 09 / 1993$

APPROVAL TYPE: SPECIAL FORM

REGULATION: SUBPART H TO 10 CFR PART 71
FEE CATEGORY: $10 B(2)$ (2)
USE (S)

USE

MAINTENANCE

PROCUREMENT REPAIR

\section{COMMENTS:}


EXPIRATION DATE : 09/30/1998

ORIG. ISSUE DATE : 09/21/1993

APPROVAL TYPE: NORMAL AND SPECIAL FORM

250 BERRYHILL ROAD, SUITE 400

COLUMBIA RS. ROBERTA SUMMERS SC 29210

COMMENTS :

REGULATION: SUBPART H TO 10 CFR PART 71

FEE CATEGORY: IOB(2)

\section{USE(S)}

USE

MAINTENANCE PROCUREMEN REPAIR

\section{DOCKETH : 07100771 \\ REVISION\#: 0}

USER NAME: LONGVIEW INSPECTION GULF COAST GROUP, INC. 101 UNDERWOOD ROAD BUILDING LAPORTE ATTN: MR. LLOYD A. GRAY
TX 77571
EXPIRATION DATE: $02 / 28 / 1999$ ORIG. ISSUE DATE: 02/02/1994 APPROVAL TYPE: SPECIAL FORM

REGULATION: SUBPART H TO 10 CFR PART 71
FEE CATEGORY: $10 B(2)$
USE(S)

USE

MAINTENANCE PROCUREMEN REPAIR

COMMENTS :

DOCKETH : 07100772

REVISION\#: 1

USER NAME: DEPT. OF AIR FORCE

HO AFTAC/SE

1030 SOUTH HIGHWAY AIA

PATRICK AFB

COMMENTS:
EXPIRATION DATE: $10 / 31 / 1998$
ORIG. ISSUE DATE: $10 / 06 / 1993$

APPROVAL TYPE: SPECIAL FORM

REGULATION: SUBPART H TO 10 CFR PART 71
FEE CATEGORY: $10 B(2)$

\begin{tabular}{l} 
USE(S) \\
\hline DESIGN \\
USE \\
MAINTENANCE \\
PROCUREMENT \\
REPAIR
\end{tabular}


REVISION\# : 0

USER NAME: PACIFIC RIM CONSULTING AND INSPECTION CORP 3375 KOAPAKA STREET, NO.. H-465

HONOLULU

COMMENTS:

DOCKETH : 07100774

REVISION\#: 0

USER NAME: MIDWEST INDUSTRIAL X-RAY, INC.

PIO2. 7 TOX 133 AVE. NORTH

ND 58107

ATTN: MR. MICHAEL G. MANGER

D 58107

COMMENTS :

DOCKET\# : 07100775

REVISION\#: 1

USER NAME: IMPERIAL INSPECTION, INC. I13 ROYAL STREET

\section{WEST MONROE \\ ATTN: ${ }^{\text {MR. JERRY WAGGONER }}$ \\ LA 71291}

EXPIRATION DATE: 09/30/1998

ORIG. ISSUE DATE: OQ/3O/1998

APPROVAL TYPE: SPECIAL FORM

REGULATION: SUBPART H TO 10 CFR PART 71

FEE CATEGORY: 1OB(2)
USE $(\underline{S})$

USE

MAINTENANCE

PROCUREIAENT

REPAIR

COMMENTS:

EXPIRATION DATE: $12 / 31 / 1998$

APPROVAL TYPE: SPECIAL FORM

REGULATION: SUBPART H TO 10 CFR PART 71

FEE CATEGORY: 1OB(2.)

\section{USE \\ MSE \\ PROCUREMENT}

REPAIR
EXPIRATION DATE: $12 / 31 / 1998$

APPROVAL TYPE: SPECIAL FORM

REGULATION: SUBPART H TO 10 CFR PART 71 FEE CATEGORY: $10 \mathrm{~B}(2)$ $-\therefore$

IISE

MAINTENANCE

PROCUREMENT

REPAIR 
DOCKETH: 07100777

REVISIONA : 0

USER NAME: VALLEY INDUSTRIAL $X$-RAY AND INSPECTION SERVICES 3545 BOWMAN COURT

BAKERSFIELD
ATTN: MR. LARRY WILLIAMS CA 93308
EXPIRATION DATE: $12 / 31 / 1998$

ORIG. ISSUE DATE: 01/03/1994

APPROVAL TYPE: SPECIAL FORM

REGULATION: SUBPART H TO 10 CFR PART 71 FEE CATEGORY: 1OB(2)

$\operatorname{USE}(S)$

USE

MAINTENANCE PROCUREMENT REPAIR

\section{COMMENTS :}

DOCKETH : 07100778

\section{REVISION\#: 0}

USER NAME: DERBY CITY INSPECTION, INC. 4340 SANITA COURT, SUITE A LOUISVILLEE GERALD R. REAMS KY 40213 COMMENTS:

DOCKETH : 07100779

USER NAME: ULTRASONIC FIELD SERVICES, CORP. 1211 KONA DRIVE RANCHO DOMINQUEZ
ATTN: MR. WILLIAM J. DE LUCA 90220

\section{REVISION\#: 0}

EXPIRATION DATE: $01 / 31 / 1999$ ORIG. ISSUE DATE: $01 / 07 / 199$ APPROVAL TYPE: SPECIAL FORM

REGULATION: SUBPART H TO 10 CFR PART 71 FEE CATEGORY: $10 B(2)$
EXP IRATION DATE: $12 / 31 / 1998$

ORIG. ISSUE DATE: 01/03/1994

APPROVAL TYPE: SPECIAL FORM

REGULATION: SUBPART H TO 10 CFR PART 71 FEE CATEGORY: $1 O B(2)$
$\operatorname{USE}(S)$

USE PROCUREMENT REPAIR

COMMENTS: 
DOCKET\# : 07100780

REVISIONH: 0

EXP IRATION DATE: $04 / 30 / 1999$
ORIG. ISSUE DATE: $04 / 12 / 1994$

USER NAME: WESTERN INDUSTRIAL X-RAY INSPECTION COMPANY, INC. 5354 HIGHWAY 89 NORTH, P. D. BOX 468 ATTN: MR. LARRY D. WICKS

WY 82931 (1)

COMMENTS:

DOCKETH: 07100781

REVISION\#: 0

USER NAME: PROFESSIONAL QUALITY TESTING COMPANY P. O. BOX 327

NEW KENSINGTON PA 15068
ATTN: MR, JOHN C. CHABAL

PA 15068

COMMENTS:
DOCKETH: 07100782

USER NAME: INTL. RADIOGRAPHY \& INSP. SERV.

W. MELROSE LA

OKLAHOMA CITY ATTN: MR. JOHN T. CHARTER OK 76302

COMMENTS:
APPROVAL TYPE: SPECIAL FORM

REGULATION: SUBPART H TO 10 CFR PART 71 FEE CATEGORY: $1 O B(2)$

\section{USE(S)}

\section{USE}

MAINTENANCE

PROCUREMENT REPAIR
EXPIRATION DATE : 02/28/2000

ORIG. I SSUE DATE: 02/22/1995

APPROVAL TYPE: NORMAL AND SPECIAL FORM

REGULATION: SUBPART H TO 10 CFR PART 71

FEE CATEGORY: $10 B(2)$
USE (S)

USE

MAINTENANCE

PROCUREMENT

REPAIR
EXPIRATION DATE: $03 / 31 / 1999$
ORIG. ISSUE DATE : $03 / 11 / 1994$

APPROVAL TYPE: SPECIAL FORM

REGULATION: SUBPART H TO 10 CFR PART 71

FEE CATEGORY: $10 B(2)$
USE (S)

USE

MAINTENANCE

PROCUREMENT

REPAIR 
DOCKET\# : 07100783

\section{REVISION\#: 0}

USER NAME: NORTHWEST INSPECTION AND TESTING SERVICES, INC. P. O. BOX 50799

IDAHO FALLS

COMMENTS :

DOCKET\#: 07100784

REVISION\#: 0

USER NAME: HOLTEC INTERNATIONAL

2060 FAIRFAX AVENUE

CHERRY HILL
ATTN: MR. MARK SOLER

NJ 080031666

COMMENTS :

DOCKET\# : 07100786

REVISION\#: 1

USER NAME: U. S. DEPARTMENT OF ENERGY

OFFICE OF CIVILIAN RADIOACTIVE
WASTE MANAGEMENT
ATTN: MGTON. RONALD A. MILNER DC 20585
EXPIRAT ION DATE: $04 / 30 / 1999$
ORIG. I SSUE DATE: $04 / 06 / 1994$

APPROVAL TYPE: SPECIAL FORM

REGULATION: SUBPART H TO 10 CFR PART 71
FEE CATEGORY: $10 B(2)$

\begin{tabular}{l} 
USE (S) \\
\hline USE \\
MAINTENANCE \\
PROCUREMENT \\
REPAIR
\end{tabular}

EXP IRAT ION DATE : $08 / 31 / 1999$
ORIG. ISSUE DATE : $08 / 23 / 1994$

APPROVAL TYPE: NORMAL AND SPECIAL FORM

REGULATION: SUBPART H TO 10 CFR PART 71
FEE CATEGORY: $1 O B(1)$

FABR ICATION

\section{USE (S)}

USE

MAINTENANCE

ASSEMBLY

MODIFICATION

PROCUREMENT

REPAIR

TESTING
DESIGN
WASTE MANAGEMENT
WASHINGTON RONA MR. RONALD A. MILNER DC 20585

WASTE MANAGEMENT
WASHINGTON RONA MR. RONALD A. MILNER DC 20585

WASTE MANAGEMENT
WASHINGTON RONA MR. RONALD A. MILNER DC 20585

COMMENTS :
EXPIRATION DATE: $08 / 31 / 1999$
ORIG. I SSUE DATE:

APPROVAL TYPE: NORMAL AND SPECIAL FORM

REGULATION: SUBPART H TO 10 CFR PART 71

FEE CATEGORY: OTHER
USE (S)

USE

MAINTENANCE

ASSEMBLY

MOD IF ICATION

PROCUREMENT

FABRICATION

REPAIR

TESTING 
DOCKET\# : 07100787

REVISION\# : 0

USER NAME: LAFAYETTE TESTING SERVICES, INC.

LAFAYETTE

COMMENTS :
EXPIRATION DATE: 08/31/1999

APPROVAL TYPE: SPECIAL FORM

REGULATION: SUBPART H TO 10 CFR PART 71
FEE CATEGORY: 1 OB (2)
USE (S)

USE

MAINTENANCE

PROCUREMENT

REPAIR
DOCKETH : 07100791

REVISION\# : 1

USER NAME: ALARON CORPORATION

RD. \#2, BOX 2140A

WAMPUM
ATTN:
PR. JONATHAN WALLACE 16157

COMMENTS :
DOCKETH : 07100801

USER NAME: MCNDT LEASING, INC.

P.O. BOX 545

CHANNAHON IL 60410
REVISIONH: 0
EXP IRATION DATE: $12 / 31 / 1999$

ORTG. ISSUE DATE: $10 / 06 / 1994$

APPROVAL TYPE: NORMAL AND SPECIAL FORM

REGULATION: SUBPART H TO 10 CFR PART 71

FEE CATEGORY: $10 B(2)$
USE(S)

USE

MAINTENANCE

PROCUREMENT

REPAIR

COMMENTS :

EXPIRATION DATE: $01 / 31 / 2000$

ORIG. ISSUE DATE: 01/09/1995

APPROVAL TYPE: SPECIAL FORM

REGULATION: SUBPART H TO 10 CFR PART 71
FEE CATEGORY: $10 B(2)$
USE (S)

USE

MAINTENANCE PROCUREMENT REPAIR 
REVISION\# : 0

USER NAME: NORCAL TESTING, INC.

P 707 . BNTX 238 ERTR DRIVE, STE F

ATTN: MS. ROSE M. FINKENBINDER

945330023

COMMENTS :

DOCKETH : 07100804

REVISION\#: 0

USER NAME: SIERRA NUCLEAR CORPORATION 620 COLONIAL PARK DRIVE

ROSWELL ATTN: MR. GEORGE N. DIXON, JR. 30075
EXPIRATION DATE: $01 / 31 / 2000$

APPROVAL TYPE: SPECIAL FORM

REGULATION: SUBPART H TO 10 CFR PART 71

FEE CATEGORY: $10 B(2)$
USE

MAINTENANCE PROCUREMENT REPAIR
EXPIRATION DATE: $06 / 30 / 2000$
ORIG. ISSUE DATE: $06 / 06 / 1995$

APPROVAL TYPE: NORMAL AND SPECIAL FORM

REGULATION: SUBPART H TO 10 CFR PART 71 FEE CATEGORY: $1 O B(1)$
USE (S)

USE MAINTENANCE ASSEMBLY MODIFICATION PROCUREMENT FABRICATION REPAIR TESTING

COMMENTS :

DOCKET\# : 07100805

REVISION\#: 0

USER NAME: OCEAN STATE TESTING, INC. 265 CONWAY STREE

NORTH KINGSTOWN
ATTN: MR. FRANK L. MCCANN RI 02852
EXPIRATION DATE: 02/28/2000

APPROVAL TYPE: SPECIAL FORM

REGULATION: SUBPART H TO 10 CFR PART 71 FEE CATEGORY: $1 O B(2)$
USE (S)

USE

MAINTENANCE

PROCUREMENT REPAIR

COMMENTS: 
EXPIRATION DATE: $04 / 30 / 2000$ ORIG. ISSUE DATE: 04/20/1995

USER NAME: MARYLAND $q_{\dot{B}}$ C. LABORATORIES, INC.

BELCAMP ${ }_{\text {ATTN: }}$ MR. BRUCE E. DEWEY MD 21017

APPROVAL TYPE: SPECIAL FORM

REGULATION: SUBPART H TO 10 CFR PART 71
USE (S)

USE MAINTENANCE PROCUREMENT REPAIR

COMMENTS :

DOCKET\# : 07100808

REVISION\# : 0

USER NAME: DELTA AIR LINES, INC., DEPARTMENT 521 TOC-1 HARTSFIELD ATLANTA INTERNATIONAL AIRPORT ATLANTA ATTN: MR. RAYMOND L. WORLEY 303206001

\section{COMMENTS :}

DOCKET\# : 07100810

REVISION\# : 1

USER NAME: ENVIRONMENTAL TECHNOLOGIES, INC.

\footnotetext{
1211-D 29TH STREET. NW

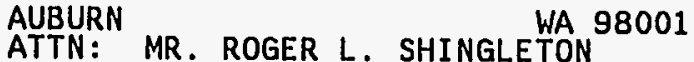

AUBURN
ATTN:
}

EXPIRATION DATE: $04 / 30 / 2000$

APPROVAL TYPE: SPECIAL FORM

REGULATION: SUBPART H TO 10 CFR PART 71 FEE CATEGORY: $10 B(2)$
USE (S)

USE

MAINTENANCE

PROCUREMENT

REPAIR

\section{COMMENTS :}

EXPIRATION DATE : $05 / 31 / 2000$
ORIG. ISSUE DATE : $05 / 17 / 1995$

APPROVAL TYPE: NORMAL AND SPECIAL FORM

REGULATION: SUBPART H TO 10 CFR PART 71
FEE CATEGORY: $10 B(1)$

\begin{tabular}{l}
\multicolumn{1}{c}{ USE(S) } \\
\hline USE \\
MAINTENANCE \\
ASSEMBLY \\
MODIFICATION \\
PROCUREMENT \\
FABRICATION \\
REPAIR \\
TESTING \\
DESIGN
\end{tabular}


DOCKET\# : 07100813

REVISION\# : 0 USER NAME: IOWA STATE UNIVERSITY OF SCIENCE AND TECHNOLOGY

AMES IA 500112241

COMMENTS:
EXPIRATION DATE: $05 / 31 / 2000$
ORIG. I SSUE DATE : $05 / 23 / 1995$

APPROVAL TYPE: NORMAL AND SPECIAL FORM

REGULATION: SUBPART H TO 10 CFR PART 71
USE(S)

USE

MAINTENANCE

PROCUREMEN REPAIR

DOCKETH : 07100817

REVISION\#: 0 USER NAME: MESCALERO ENVIRONMENTAL, INC.
P. O. BOX 176

MESCALERO

ATTN: MR. FRED PESO

NM 88340

REVISION\#: 0

DOCKETH : 07100818

USER NAME: ENTEC CONSULTANTS, INC.

925 BROADWAY

ALBANY RY 12207
EXPIRATION DATE: $07 / 31 / 2000$
ORIG. ISSUE DATE : $07 / 21 / 1995$

APPROVAL TYPE: NORMAL AND SPECIAL FORM

REGULATION: SUBPART H TO 10 CFR PART 71

\begin{tabular}{l} 
USE(S) \\
\hline ASSEMBLY \\
MODIFICATION \\
FABRICATION \\
TESTING \\
DESIGN \\
USE \\
MAINTENANCE \\
PROCUREMENT \\
REPAIR
\end{tabular}
PRECUREMEN

COMMENTS EXPIRATION DATE: $08 / 31 / 2000$

APPROVAL TYPE: SPECIAL FORM

REGULATION: SUBPART H TO 10 CFR PART 71
$\operatorname{USE}(S)$

USE MAINTENANCE PROCUREMENT REPAIR

COMMENTS : 
DOCKETH : 07100821

REVISION\#: 0 USER NAME: PENSACOLA TESTING LABORATORIES, INC.

PENSACOLA PATRICK A. WHEELEL 32503

COMMENTS:

DOCKETH : 07100824

REVISION\#: 0

USER NAME: $U$. $S$. DEPARTMENT OF THE NAVY CHARLESTON NAVAL SHIPYARD

1351 FIRST STREET

ATTN: MR. MALCOLM J. HURSEY

294082020

COMMENTS :

DOCKETH : 07100825

REVISION\# : 0

USER NAME: OCEAN STATE TECHNICAL SERVICES

2440 MENDON ROAD

\footnotetext{
CUMBERLAND

CUMBERLAND
}

EXPIRATION DATE: $07 / 31 / 2000$

APPROVAL TYPE: SPECIAL FORM

REGULATION: SUBPART H TO 10 CFR PART 71

FEE CATEGORY: $10 B(2)$

\section{USE(S)}

USE

MAINTENANCE

PROCUREMENT

REPAIR

COMMENTS:

EXPIRATION DATE: $02 / 28 / 1996$
ORIG. ISSUE DATE : $08 / 18 / 1995$

APPROVAL TYPE: SPECIAL FORM

REGULATION: SUBPART H TO 10 CFR PART 71

FEE CATEGORY: EDUC
EXPIRATION DATE: $08 / 31 / 2000$

APPROVAL TYPE: SPECIAL FORM

REGULATION: SUBPART H TO 10 CFR PART 71

FEE CATEGORY: $10 B(2)$
USE (S)

USE

MAINTENANCE

PROCUREMENT

REPAIR
USE(S)

USE

СОМЕТS:


DOCKETH : 07100826

REVISIONH : 1

USER NAME: $U$. S. DEPARTMENT OF THE NAVY-NISE WEST FACILITY PSC 473 BOX 5 YOKOSUKA, JAPAN ATTN: LT. C. GEDO

963492901

COMMENTS :
REVISION\# : 0

USER NAME: SOLUSS SCHALL 1441 PARK TEN BOULEVARD

HOUSTON
ATTN : RADIATION SAFETY OFFICER

COMMENTS :

DOCKETH : 07100829

REVISION\#: 1

USER NAME: PRIVATE FUEL STORAGE LIMITED LIABILITY COMPANY

P. O. BOX C4010

LACROSSE ATTN: JOHN D. PARKYN WI 546024010

USER
EXP IRATION DATE: $05 / 31 / 1996$

ORIG. ISSUE DATE: 08/18/1995

APPROVAL TYPE: SPECIAL FORM

REGULATION: SUBPART H TO 10 CFR PART 71
FEE CATEGORY: $10 B(2)$
USE

$\operatorname{USE}(S)$
EXPIRATION DATE: 09/30/2000

ORIG. ISSUE DATE: $09 \% 08 / 1995$

REGULATION: SUBPART H TO 10 CFR PART 71

FEE CATEGORY: 1OB(2)

USE(S)
USE
MAINTENANCE
ROCUREMENT

PROCUREMENT REPAIR
EXPIRATION DATE: $09 / 30 / 2001$
ORIG. ISSUE DATE : $11 / 03 / 1995$

APPROVAL TYPE: NORMAL AND SPECIAL FORM

REGULATION: SUBPART H TO 10 CFR PART 71
FEE CATEGORY: $10 B(2)$
USE (S)

USE

MAINTENANCE PROCUREMENT REPAIR

COMMENTS: 
DOCKETH : 07100830

REVISION\# : 0

USER NAME: ITT INSPECTION - TECH TECHNICIANS, INC. P. 0. BOX 15135

KANSAS CITY ATTN: MR. C. V. ROUDEBUSH MO 64106

COMMENTS :
REVISIONH : 0

USER NAME: GEORGIA INSTITUTE OF TECHNOLOGY NEELY NUGLEAR RESEARCH CENTER 900 ATLANTIC DRIVE ATLANTA ATTN: DR. R. A. KARAM

GA 303320425
EXP IRATION DATE: $10 / 31 / 2000$

ORIG. ISSUE DATE : 10/26/1995

APPROVAL TYPE: SPECIAL FORM

REGULATION: SUBPART H TO 10 CFR PART 71

FEE CATEGORY: 10B(2)
USE (S)

USE

MAINTENANCE

PROCUREMENT

REPAIR

\section{COMMENTS:}

DOCKETH : 07100832

REVISION\# : 1

USER NAME: UNITED STATES ENRICHMENT CORPORATION 6903 ROCKLEDGE DRIVE

BETHESDA MD 20817

ATTN: MS. BETH DARROUGH
EXPIRATION DATE: $10 / 31 / 2000$

APPROVAL TYPE: NORMAL AND SPECIAL FORM REGULATION: SUBPART H TO 10 CFR PART 71
FEE CATEGORY: EDUC
USE (S)

USE

MAINTENANCE

PROCUREMENT

REPAIR

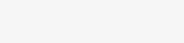

\author{
EXP IRATION DATE: 03/31/2001 \\ APPROVAL TYPE: NORMAL AND SPECIAL FORM \\ REGULATION: SUBPART H TO 10 CFR PART 71
FEE CATEGORY: $10 B(1)$
}

\begin{tabular}{l}
\multicolumn{1}{c}{ USE(S) } \\
\hline USE \\
MAINTENANCE \\
ASSEMBLY \\
MODIFICATION \\
PROCUREMENT \\
FABRICATION \\
REPAIR \\
TIESTING \\
DESIGN
\end{tabular}


REVISION\#: 0 USER NAME: REINHART \& ASSOCIATES, INC.

AUSTIN MR JOHN E, FORBIS TX 78766

EXPIRATION DATE: $11 / 30 / 2000$

ORIG. I SSUE DATE: $11 / 15 / 1995$

APPROVAL TYPE: SPECIAL FORM

REGULATION: SUBPART H TO 10 CFR PART 71
USE(S)

USE

MAINTENANCE PROCUREMENT REPAIR

COMMENTS:

DOCKETH : 07100834

REVISIONH: 0

USER NAME: UNIVERSITY OF MINNESOTA

410 CHURCH STREET, S.E.

MINNEAPOLIS

COMMENTS:

DOCKETH : 07100835

REVISIONH: 0

USER NAME: NORTH ATLANTIC ENERGY SERVICE .CORPORATION

P. O. BOX 300

AEABROOK ATTN: TED C. FEIGENBAUM 03874

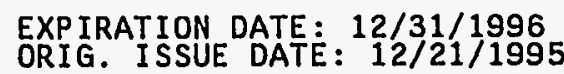

APPROVAL TYPE: SPECIAL FORM

FEE CATEGORY: EDUC
REGULATION: SUBPART H TO 10 CFR PART 71

USE(S)

EXP IRATION DATE: $11 / 30 / 2000$

ORIG. ISSUE DATE: $12 / 06 / 1995$

APPROVAL TYPE: SPECIAL FORM

REGULATION: APPENDIX B TO 10 CFR PART 50

FEE CATEGORY: $10 B(2)$
$\operatorname{USE}(S)$

USE

MAINTENANCE REPAIR

\section{COMMENTS:}




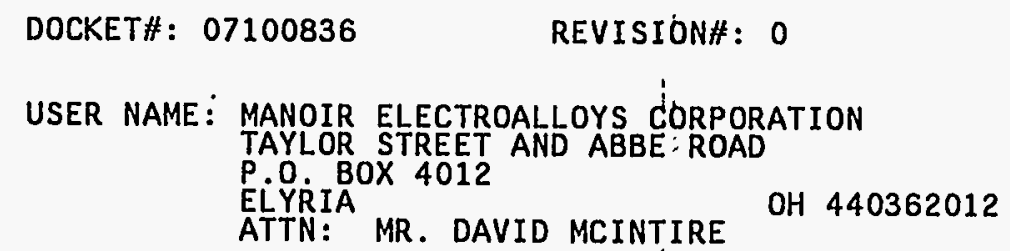

USER NAME: MANOIR ELECTROALLOYS CORPORATION TAYLOR STREET AND ABBE: ROAD P.O. BOX 4012 ATTN: MR. DAVID MCINTIRE

COMMENTS:

EXP IRATION DATE: $12 / 31 / 2000$
ORIG. ISSUE DATE : $01 / 05 / 1996$

APPROVAL TYPE: SPECIAL FORM

REGULATION: SUBPART H TO 10 CFR PART 71
FEE CATEGORY: $10 B(2)$
USE S)

USE

MAINTEN/NCE PROCUREIIENT REPAIR

DOCKETH : 07100837

REVISIONH : 0 USER NAME: ICF KAISER HANFORD COMPANY RICHLAND
ATTN: MR WILLIAM S. ROE

WA 99352 . COMMENTS:

DOCKET\# : 07100838

REVISION\#: 0

USER NAME: PACIFIC TESTING LABORATORIES 3257 16TH AVENUE WEST

AEATTLE ${ }_{\text {MR. EMERY E. ROBERTS }}$ WA 98119
EXPIRATION DATE: 01/31/2001

ORIG. I SSUE DATE: 01/05/1996

REGULATION: SUBPART H TO 10 CFR PART 71

FEE CATEGORY: $1 O B(2)$
- 
EXPIRATION DATE: $01 / 31 / 2001$ USER NAME: DECISIVE TESTING, INC.

SAN DIEGO CA 92105
APPROVAL TYPE: SPECIAL FORM

ATTN: MR. MICHAEL MAY

CA 92105

REGULATION: SUBPART H TO 10 CFR PART 71

COMMENTS :

DOCKETH : 07100840

REVISION\#: 0

USER NAME: INDUSTRIAL MARINE TESTING LABORATORY 3167 COMMERCIAL STREET

SAN DIEGO SAMUEL A. BOYKIN CA 921131426

COMMENTS :

DOCKETH : 07100841

REVISIONA : 0

USER NAME: TIDELANDS TESTING, INC

1120 HOOVER AVENUE, SUITE D

NATIONAL CITY

CA 91950
EXP IRATION DATE: $01 / 31 / 2001$
ORIG. ISSUE DATE: $01 / 17 / 1996$

APPROVAL TYPE: SPECIAL FORM

REGULATION: SUBPART H TO 10 CFR PART 71
FEE CATEGORY: $10 B(2)$
USE(S)

USE

MAINTENANCE PROCUREMEN REPAIR

\section{COMMENTS:}

EXPIRATION DATE: 01/31/2001

APPROVAL TYPE:' SPECIAL FORM

REGULATION: SUBPART H TO 10 CFR PART 71

FEE CATEGORY: $10 B(2)$
USE $(S)$

USE

MAINTENANCE PROCUREMENT REPAIR 
DOCKET\# : 07100842

REVISIONH: 0 USER NAME: PANHANDLE NDT AND INSPECTION

BORGER MR. ORVIL COUCH

COMMENTS :

DOCKET\# : 07100843

REVISION\#: 0

USER NAME: TESTING SERVICES \& INSPECTION, INC. 3030 MAIN STREET

SAN DIEGO CA 92113

ATTN: MR. JUAN R. DIAZ

COMMENTS:

DOCKET\# : 07100844

REVISION\# : 0

USER NAME: UNIVERSITY OF MISSOURI

1870 MINER CIRCLE

ROLLA MR. HATEM KHOUAJA

M0 654090630
EXPIRATION.DATE: $01 / 31 / 2001$

APPROVAL TYPE: SPECIAL FORM

REGULATION: SUBPART H TO 10 CFR PART 71
FEE CATEGORY: $10 B(2)$
$\operatorname{USE}(S)$

USE

MAINTENANCE

PROCUREMENT

REPAIR
EXPIRATION DATE: 01/31/2001

APPROVAL TYPE: SPECIAL FORM

REGULATION: SUBPART H TO 10 CFR PART 71

FEE CATEGORY: $1 O B(2)$

USE (S)
INTENANCE
OCUREMENT
PAIR

PROCUREMENT

REPAIR

\section{COMMENTS :}

EXPIRATION DATE: $04 / 30 / 1998$

APPROVAL TYPE: SPECIAL FORM

REGULATION: SUBPART H TO 10 CFR PART 71

FEE CATEGORY: EDUC
USE (S)

USE

MAINTENANCE

PROCUREMENT

REPAIR 
DOCKETH : 07100845

REVISION\# : 0

USER NAME: WELLESLEY COLLEGE

DEPT. OF BIOLOGICAL SCIENCES

106 CENTRAL STREET

WELLESLEY

ATTN: T. KAYE PETERMAN

MA 021818283

COMMENTS:

DOCKET\# : 07100846

REVISION\#: 0

USER NAME: WEST VIRGINIA UNIVERSITY HOSPITALS

P. O. BOX 8150

MORGANTOWN ATTN: MR. ERIC RAUDENBUSH WV 265068150

COMMENTS :

DOCKETH : 07100847

REVISION\#: 0

USER NAME: WILLIAMS COLLEGE

DEPARTMENT OF CHEMI STRY

WILLIAMSTOWN
EXPIRATION DATE: $02 / 28 / 1997$

ORIG. ISSUE DATE: 02/21/1996

APPROVAL TYPE: SPECIAL FORM

USE(S)

USE

REGULATION: SUBPART H TO 10 CFR PART 71

FEE CATEGORY: EDUC
EXPIRATION DATE:
ORIG. ISSUE DATE:
O2/22/1997

APPROVAL TYPE: SPECIAL FORM

REGULATION: SUBPART H TO 10 CFR PART 71

FEE CATEGORY: $10 B(2)$
USE

USE(S)

XP IRATION DATE: 04/30/1997

APPROVAL TYPE: SPECIAL FORM

$\operatorname{USE}(S)$

USE

REGULATION: SUBPART H TO 10 CFR PART 71

FEE CATEGORY: EDUC 
DOCKET\# : 07100848

REVISION\#: 0

USER NAME: EL PASO INSPECTION P. O. BOX 12386 904 TONY LAMA STREET ATTN: MR. BOBBY S. CARR

\section{COMMENTS :}

\section{DOCKETH : 07100849}

REVISION\#: 0

USER NAME: UNIVERSITY OF MISSOURI

2411 HOLMES STREET

KANSAS CITY MILIAM J. FIELOS 641082792

COMMENTS :

DOCKETH : 07100850

REVISION\# : 0

USER NAME: COLLEGE OF WILLIAM AND MARY

WILLIAMSBURG 231873540

\section{COMMENTS :}

EXPIRATION DATE: $04 / 30 / 2001$

APPROVAL TYPE: SPECIAL FORM

REGULATION: SUBPART H TO 10 CFR PART 71
FEE CATEGORY: $10 B(2)$
USE (S)

USE

MAINTENANCE PROCUREMENT REPAIR
EXPIRATION DATE: $04 / 30 / 1997$
ORIG. ISSUE DATE: 04/22/199

APPROVAL TYPE: SPECIAL FORM

REGULATION: SUBPART H TO 10 CFR PART 71

FEE CATEGORY: EDUC
USE (S)

USE
EXPIRATION DATE: $04 / 30 / 1997$
ORIG. ISSUE DATE: $04 / 24 / 1996$ APPROVAL TYPE: SPECIAL FORM

REGULATION: SUBPART H TO 10 CFR PART 71

REG CATEGORY: EDUC
USE (S) 
DOCKETH : 07100851

\section{REVISION\#: 0} USER NAME: MICHIGAN TECHNOLOGICAL UNIV. HOUGHTON
EXPIRATION DATE: $11 / 30 / 1996$

ORIG. ISSUE DATE: 06/10/1996

APPROVAL TYPE: SPECIAL FORM

REGULATION: SUBPART H TO 10 CFR PART 71 FEE CATEGORY: EDUC

COMMENTS:

DOCKETH: 07100853

REVISION\#: 0

USER NAME: LAUREL TECHNOLOGIES, INC.

15508 PARK HALL COURT

LAUREL MS. VICTORIA A. BORLAND 20707
EXPIRATION DATE: $07 / 31 / 2001$
ORIG. I SSUE DATE: $07 / 09 / 1996$

APPROVAL TYPE: NORMAL AND SPECIAL FORM

REGULATION: SUBPART H TO 10 CFR PART 71
FEE CATEGORY: $1 O B(1)$
USE

$\operatorname{USE}(S)$

COMMENTS :

DOCKETH : 07100854

REVISIONA : 0

USER NAME: $\begin{aligned} & X \text {-CEL NDE } \\ & \text { P.O. INC. }\end{aligned}$

- ODESSA ATTN: MR. RICHARD W. SPENCER
EXPIRATION DATE: $05 / 31 / 2001$
ORIG. ISSUE DATE:

APPROVAL TYPE: SPECIAL FORM

REGULATION: SUBPART H TO 10 CFR PART 71

FEE CATEGORY: $10 B(2)$
USE(S)

USE

ASSEMBLY

MOD IFICATION

PROCUREMENT

FABRICATION

REPAIR

TESTING

COMMENTS : 
DOCKETH : 07100855

REVISIONH: 0

USER NAME: ELITE INSPECTION SERVICE_CO., INC. 725 COLONIAL DRIVE

$$
\begin{aligned}
& \text { HOBART } \\
& \text { ATTN: MR. JOHN A. SMITH IN } 46342
\end{aligned}
$$

COMMENTS :

DOCKET\# : 07100856

REVISION\# : 0

USER NAME: CROFT ASSOCIATES LIMITED 82 NORTH CULHAM ESTATE

OXFORDSHIRE, UK OX14 3GY

ATTN: MR. TREVOR N. WALKER
EXPIRATION DATE: $05 / 31 / 2001$
ORIG. ISSUE DATE: $05 / 30 / 1996$

APPROVAL TYPE: SPECIAL FORM

REGULATION: SUBPART H TO 10 CFR PART 71

FEE CATEGORY: $10 B(2)$
USE(S)

\section{USE}

MAINTENANCE

PROCUREMENT

REPAIR

\section{COMMENTS :}

EXPIRATION DATE: 06/30/2001

APPROVAL TYPE: NORMAL AND SPECIAL FORM

REGULATION: SUBPART H TO 10 CFR PART 71

FEE CATEGORY: $10 B(1)$
USE(S)

USE

MAINTENANCE

ASSEMBLY Y

MODIFICATION

PROCUREMENT

FABRICATION

REPAIR

TESTING
EXPIRATION DATE: $06 / 30 / 1997$
ORIG. ISSUE. DATE : 06/10/1996

APPROYAL TYPE: SPECIAL FORM

REGULATION: SUBPART H TO 10 CFR PART 71
FEE CATEGORY: EDUC
ENVIRONMENTAL HEALTH \& SAFETY

135 COLLEGE STREET

ATTN: MS. AGNES BARLOW

CT 065102411

COMMENTS : 
DOCKETH : 07100858

REVISION\# : 0

USER NAME: STEVENS INSTITUTE OF TECHNOLOGY CASTLE POINT ON THE HUDSON

HOBOKEN ATTN: STEVEN SYLVESTER $^{\text {NJ } 07030}$

COMMENTS:
EXPIRATION DATE: $12 / 31 / 1996$ ORIG. ISSUE DATE: 07/02/1996

APPROVAL TYPE: SPECIAL FORM

REGULATION: SUBPART H TO 10 CFR PART 71

FEE CATEGORY: EDUC

\section{DOCKET\# : 07100859 REVISION\#: 0}

USER NAME: UNIVERSITY OF TULSA

DEPARTMENT OF PHYSICS

600 SOUTH COLLEGE AVENUE

ATTN: DR. ALLEN R. SOLTOW

COMMENTS :

DOCKETH : 07100860

REVISION\#: 0

USER NAME: TECHNOLOGY PLUS, INC.

P. 0. BOX 14119 NORTH 44 TH STREET

GRAND FORKS

ATTN: MR. DENNIS E. HANSON
ND 582084119

COMMENTS :
EXP IRATION DATE: $12 / 31 / 1996$
ORIG. ISSUE DATE : $07 / 02 / 1996$

APPROVAL TYPE: SPECIAL FORM

REGULATION: SUBPART H TO 10 CFR PART 71

FEE CATEGORY: EDUC
USE

USE (S) 
DOCKET\#: 07100861

REVISION\#: 0

USER NAME: BENEDICT COLLEGE, DEPT. OF BIOLOGICAL AND PHYSICAL SCIÉNCES HARDEN AND BLANDING STREETS COLUMBIA SC 29204 ATTN: MR. RAGHBIR SINGH

COMMENTS :

DOCKET\# : 07100862

\section{REVISION\#: 0}

USER NAME: BIONOMICS, INC.

KINGSTON

ATTN: MR. PAUL IKE NIPPER
EXPIRATION DATE : $01 / 31 / 1997$
ORIG. ISSUE DATE: $07 / 26 / 1996$

APPROVAL TYPE: SPECIAL FORM

REGULATION: SUBPART H TO 10 CFR PART 71 FEE CATEGORY: EDUC
USE
COMMENTS :
DOCKET\# : 07100863

REVISION\#: 0

USER NAME: INSPECTION SERVICE INC.

KENNEWICK WA 99336
EXPIRATION DATE: 01/31/1997

ORIG. I SSUE DATE: 08/05/1996

APPROVAL TYPE: SPECIAL FORM

REGULATION: SUBPART H TO 10 CFR PART 71

FEE CATEGORY: EDUC
USE (S)
ATTN: MR. TONY M. MARTIN

EXPIRATION DATE : 08/31/2001

APPROVAL TYPE: SPECIAL FORM

REGULATION: SUBPART H TO 10 CFR PART 71

FEE CATEGORY: $1 O B(2)$
$\operatorname{USE}(S)$

USE

MAINTENANCE PROCUREMENT

COMMENTS : 


\section{DOCKET\# : 07100864}

REVISION\#: 0

USER NAME: ADAMS INDUSTRIAL SERVICES, INC.

1885 GRAND BAY DRIVE

VIRGINIA BEACH

ATTN: MR. THOMAS D. ADAMS
EXPIRATION DATE: 09/30/2001

APPROVAL TYPE: SPECIAL FORM

REGULATION: SUBPART H TO 10 CFR PART 71 FEE CATEGORY: $10 B(2)$

\section{USE(S)}

USE

MAINTENANCE

PROCUREMENT

REPAIR

COMMENTS: 
07100472

ABC TESTING INC

ATTN: MR. C A. RICHARDSON

95 FIRST STREE
BRIDGEWATER

MA 02324

07100728

ACCU-TECH EVALUATION SERVICES, INC.

ATO MR J J

RAHWAY

NJ 07065

07100864

ADAMS INDUSTRIAL SERVICES, INC.

ATAM: MR. THOMAS D. ADAMS

ATRGINIA BEACH DRIVE

A 23456

07100354

ADVANCED MEDICAL SYSTEMS, INC.

ATTN: MS. SHERRY J. STEIN

GENEVA

ADVEX 07100437

ATVEX MORP.

AT FLOYD THOMPSON DRIVE

HAMPTON

VA 236661307

07100737

AGRA EARTH \& ENVIRONMENTAL, INC

ATTN : MS DEBBIE M. KEYES

3232 W. VIRGINIA AVENUE

PHOENIX

AZ 850091502

07100595

AKRON INDUSTRIAL SERVICES, INC.

ATTN: MR LESTER A. MOORE

$\mathrm{OH} 44240$ 


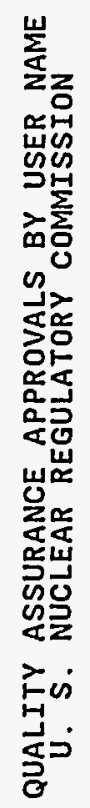

I

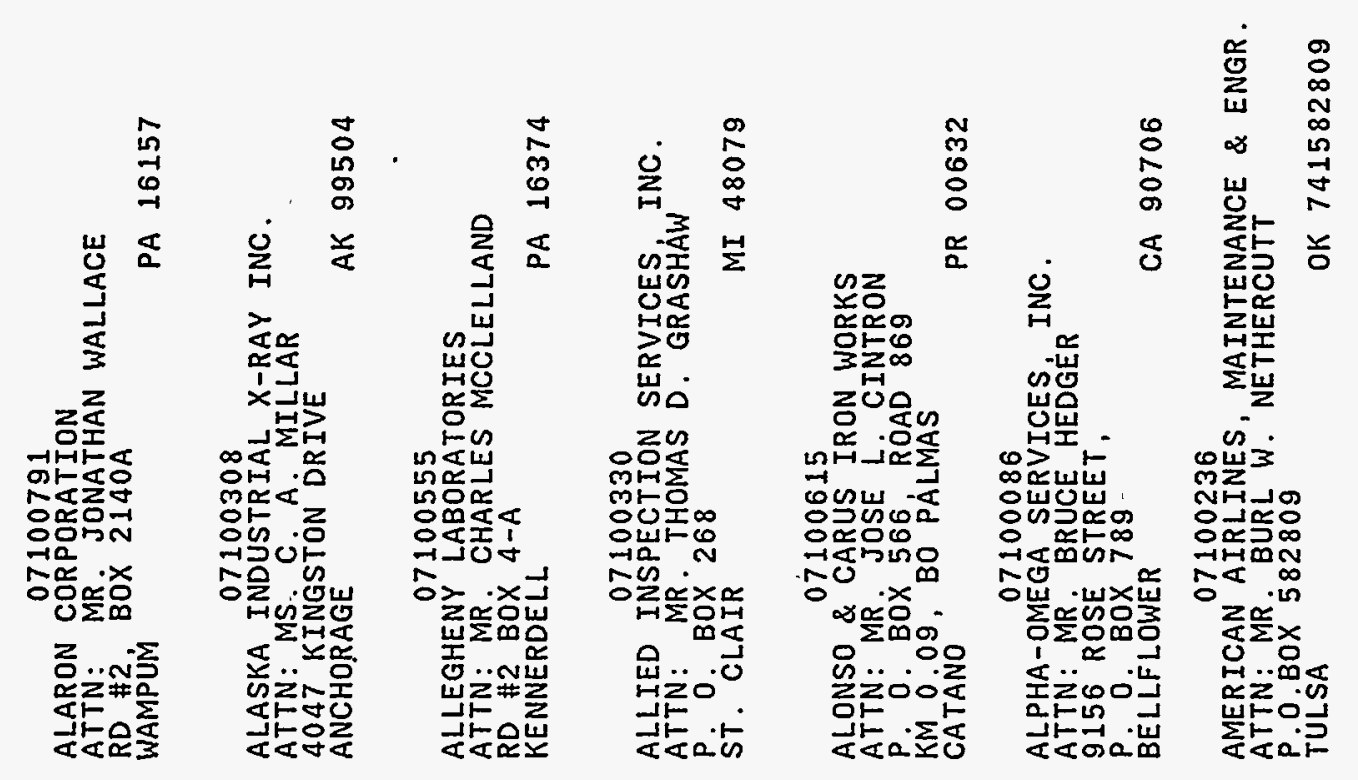


07100743

AMERICAN ASSOCIATED TESTING, INC, ATTN : MR PETER RAMIREZ

LAKE WORTH

FL 33466

07100258

AMERICAN ECOLOGY CORPORATION

ATTN: MR . ART PALMER

109 FLINT ROAD

OAK RIDGE

TN 37830

07100422

AMERICAN FOUNDRY GROUP INC.

ATTN: MR . CARL CRUCE

$1200 \mathrm{~N}$. PEORIA AVENUE

TULSA

OK 74106

07100040

AMERSHAM CORPORATION

ATTN: MS. CATHLEEN ROUGHAN

40 NORTH AVENUE

BURLINGTON

MA 01803

07100637

ANALYTIC STRESS RELIEVING, INC

ATTN: MR STRESS DIVISION

75

VA 23237

07100001

ANEFCO INC.

BOX D. MURPHY

MOODus BOX 171

CT 06469

ANVIL CORPORAT77

ATTN: MS KATHY TURNER

742 SOUTH SPRUCE STREET

BURLINGTON WA 98233 

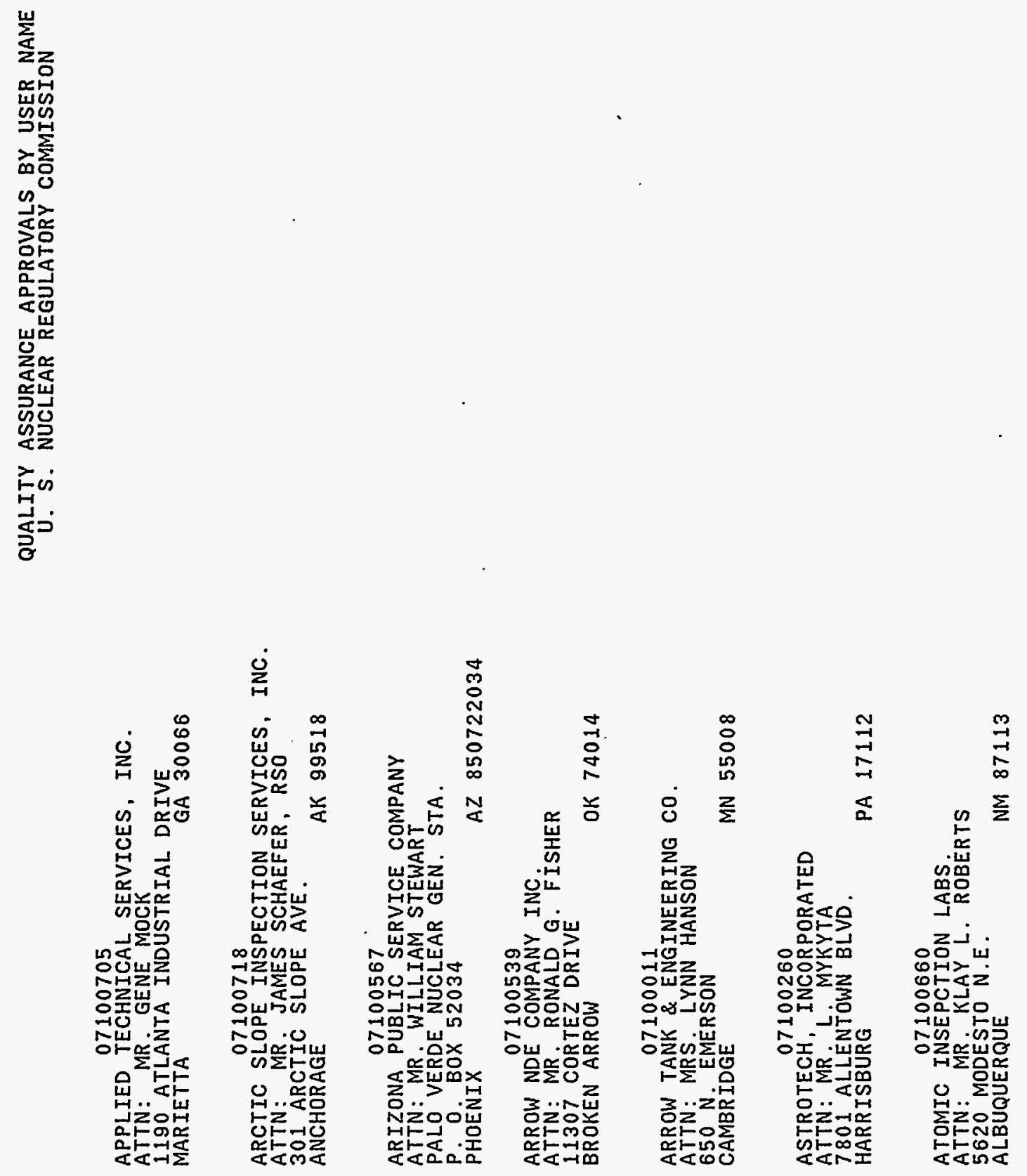
07100135

B\&W NUCLEAR ENVIRONMENTAL SERVICES, INC.

ATTN: MR $\dot{D} \cdot \mathrm{K} \cdot$. SGARLATA

VÁNDERGGRIFT

$P A 15690$

07100088

BABCOCK \& WILCOX CO NAVAL NUCLEAR FUEL DIV.

ATTN: MR. M. C. SUWALA

BOX 785

VA 24505

07100079

BABCOCK \& WILCOX CO NUCLEAR EQUIPMENT DIV.

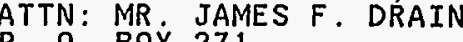

A D BOX 271

GA STIRLING AVENUE

OH 442030271

07100392

BAKER TESTING SERVICES

INC.

ATTN: MR. DONALD PALMER, RSO

98 RESERVIR PARK DRIVE

MA 02370

07100383

BALTIMORE GAS AND ELECTRIC COMPANY

ATTN: MR. R. E. DENTON

CALVERT CLIFFS NUC. PWR. PLANT

1650 CLAVERT CLIFFS PARKWAY

LUSBY 20657

07100662

BARNETT INDUSTRIAL X-RAY, INC

A ROBERT BARNET

STILLWATER
OK 74075

07100155

BATH IRON WORKS CORP

ATTN: MR. JOHN H. BERGER

700 WASHINGTON STREET

BATH

ME 04530 

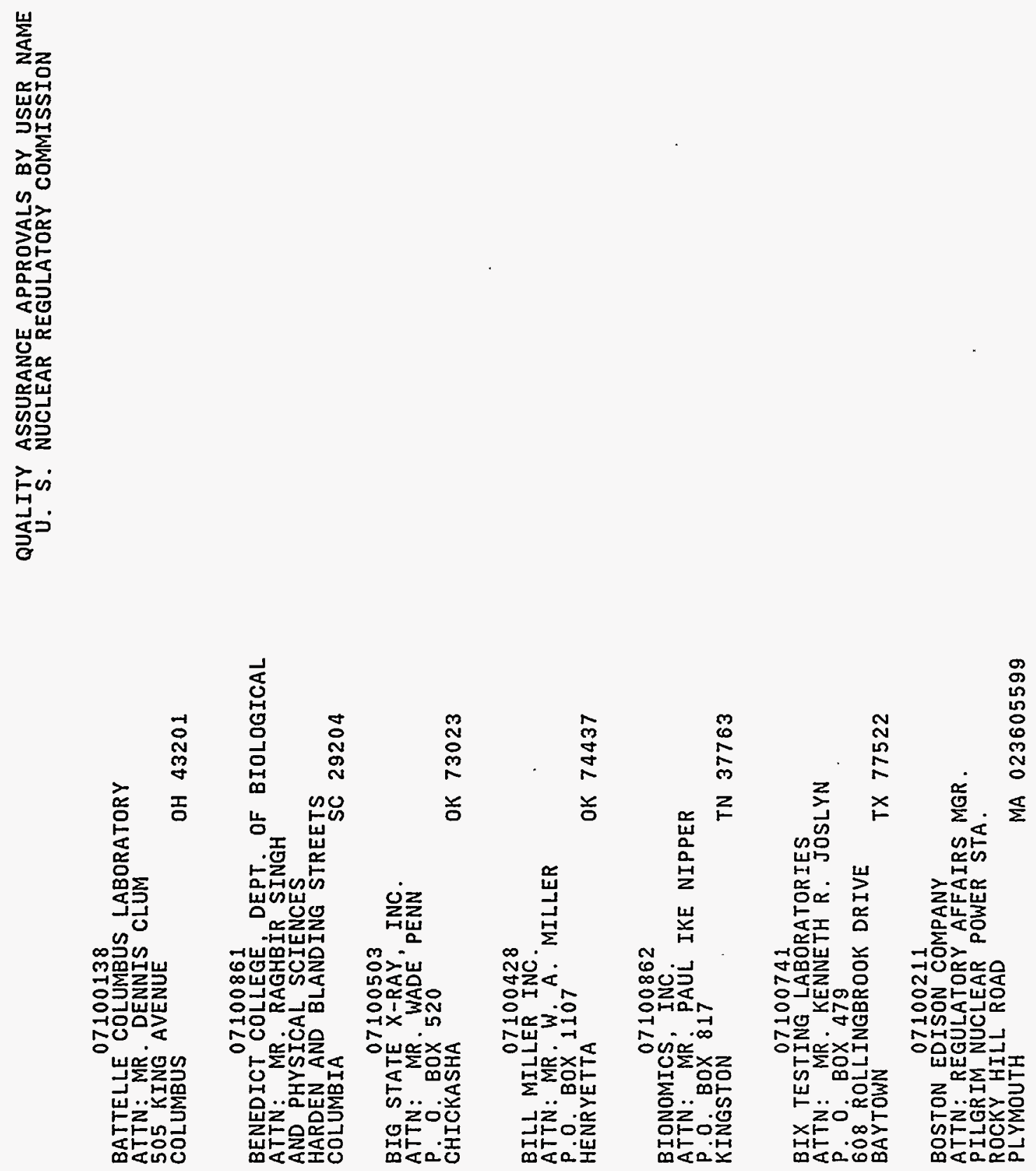
QUALITY ASSURANCE APPROVALS BY USER NAME
U. S. NUCLEAR REGULATORY COMMISSION

07100368

BRANCH RADIOGRAPHIC LABS. INC.

ATTN: MR. PETER J. BRANCH

32 SOUTH AVENUE $W$

CRANFORD

NJ 07016

07100592

BRAUN INTERTEC CORPORATION

ATTN: MR. FRED A. BOHNSACK

.0. BOX 39108

6875 WASHINGTON AVENUE SOUTH

MINNEAPOLIS

MN 55439

07100363

BRIGGS ASSOCIATES, INC.

ATTN: MR PAUL M. SKOROHOD

400 HINGHAM STREE

MA 02370

BUCKEYE 07100110

BUCKEYE STEEL CASTINGS COMPANY

ATTN: MR DELNO SEITZ

TIU PARSONS AVENUE

OH 43207

BURNLEY 07100750

BURNLEY TECHNOLOGY INC

ATTN: MR. JOHN J.'MUNRO III

PLAISTOW

NH 038651226

07100337

CALUMET TESTING SERVICE INC

ATT: MR $15 \mathrm{~J}^{\mathrm{J}}$. KEILMAN

HIGHLAND

IN 46322

07100228

CAPITAL X-RAY SERVICE

ATTN: MR. GEORGE $W$. JOHNSON

P $\mathrm{BOX} 3264$

TULSA

OK 74101 


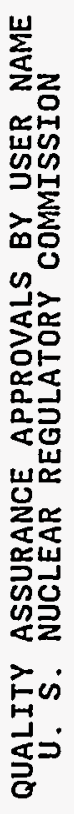

ำ

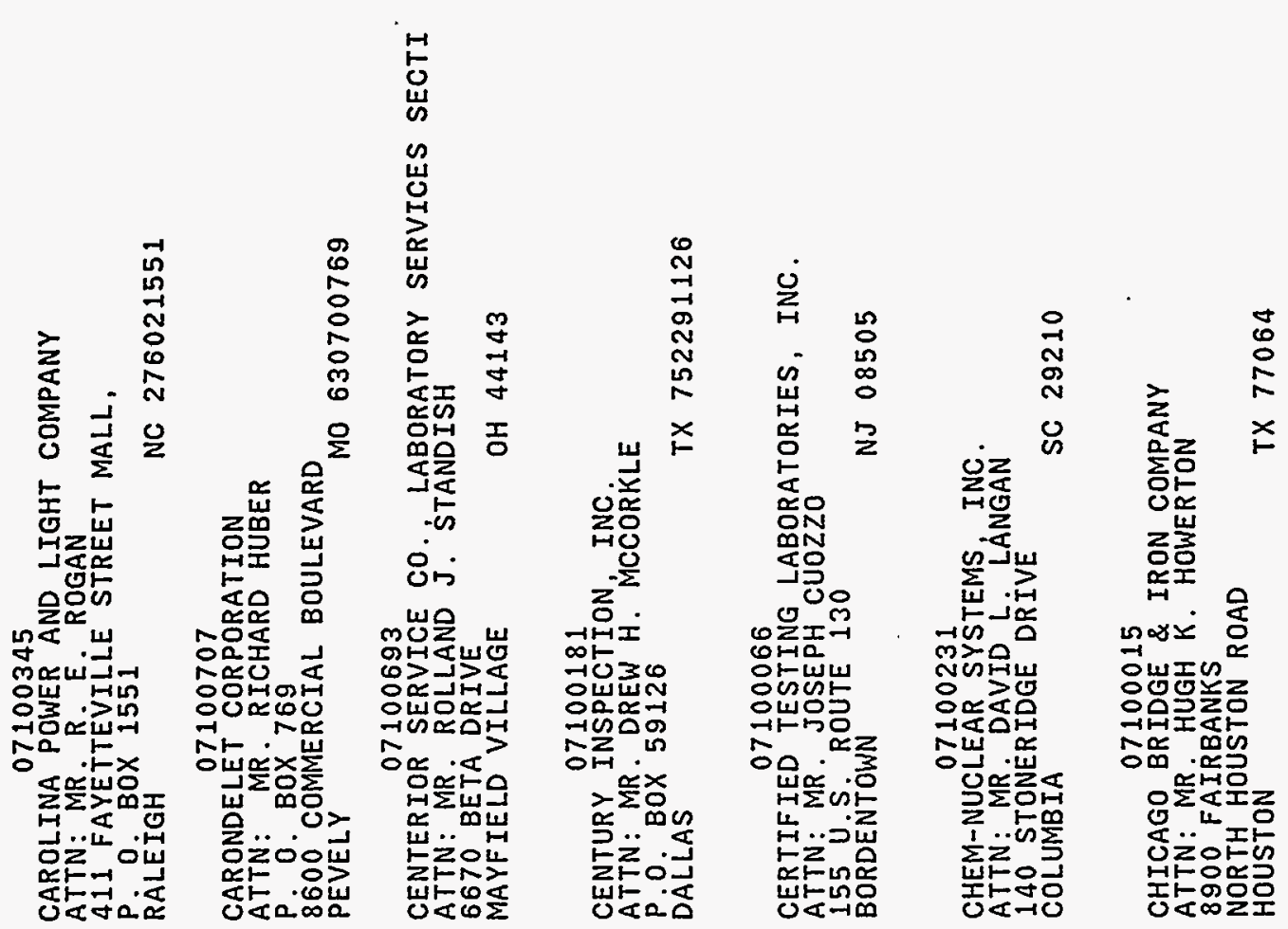




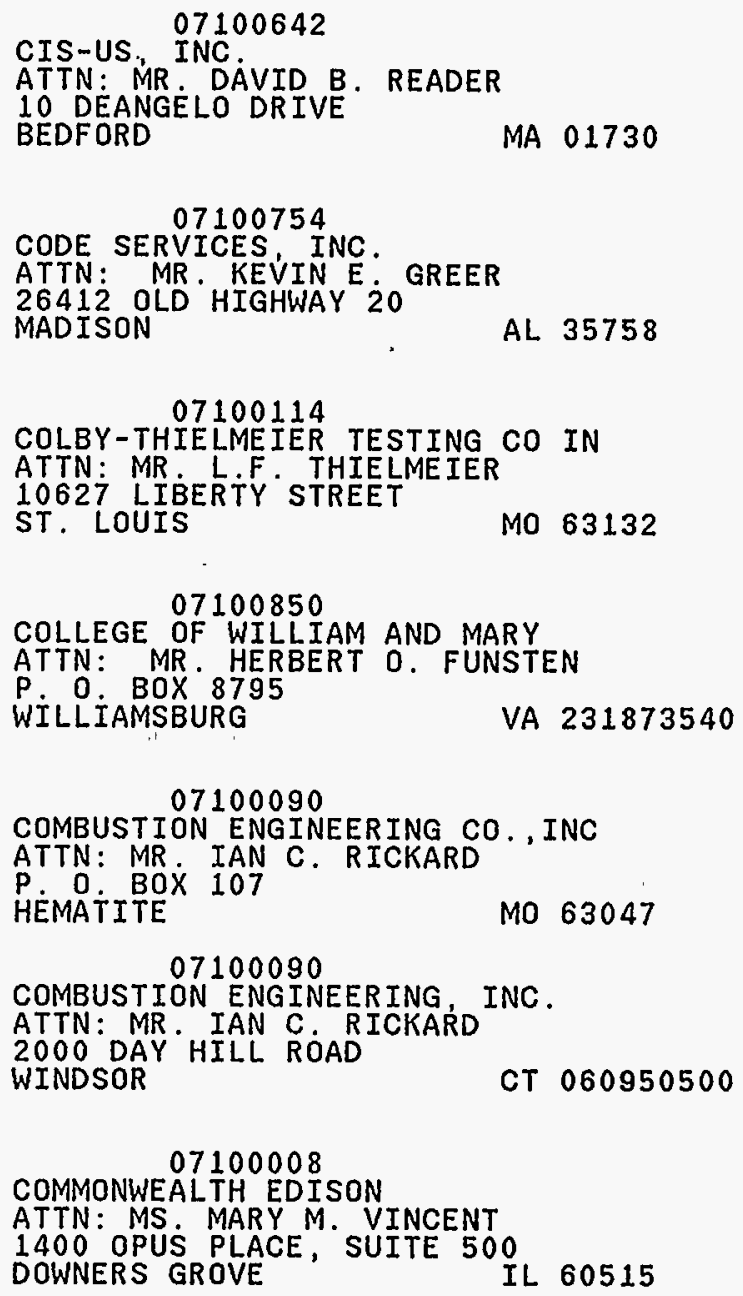

07100114

COLBY-THIELMEIER TESTING CO IN ATTN: MR. L.F. THIELMEIER

LOUIS

MO 63132

07100850

ATTN. MR WILLIAM AND MARY

T. FUNSTEN

WI U BMX 8795

VA 231873540

07100090

COMBUSTION ENG INEER ING CO., INC

ATTN: MR. IAN C RICKARD

A. BOX 107

MO 63047

07100090

COMBUSTION ENG INEER ING, INC

ATTN: MR . IAN C RICKARDI

AOO DAY HIAN CDAD

WINDSOR

CT 060950500

07100008

ATTN: MS MH EDISON

1400 OPUS MARY $M$. VINCENT

DOWNERS GROVE

IL 60515 
07100669

COMO TECH INSPECTION SERVICE

ATTN: MR R R. E. HIERSCHE

LENEXA

KS 66227

CONAM INSPECT100216

INSPECTION INC.

ATTN: MR ROBERT J SLACK

$1245 W$. NORWOOD AVÉNUE

ITASCA

IL 60143

07100207

CONNECTICUT YANK

ATTN: RADIATION SAFETY OFFICER

RFD \#1 P P O . BOX $127 E$

CT 06424

CONSOLIDAT100069

CONSOLIDATED EDISON COMPANY

ATTN: MR . STEPHEN QUINN
BROADWAY \& BLEAKLEY AVE.

BUCHANAN

NY 10511

07100627

CONSOLIDATED NDE, INC

ATTN: MR BRUCE $R$. BALLARD

6 WOODBRIDGE AVENUE,

P. 0 BOX 593

NJ 07095

07100325

CONSUMERS POWER COMPANY

ATTN: MR. THOMASS P. NEAL

PALISADES NUCLEAR PLANT

27780 BLUE STAR HWY.

COVERT

MI 49043

07100727

CONTINENTAL AIRLINES

. ROHRIG

A25OEAST SMITH ROAD

CO 80207 
07100625

COPES-VULCAN, INC

ATTN: MR F 'R. PORTER

MÁRTIN AND RICE AVE.

LAKE CITY

PA 16423

07100558

CRAMER \& LINDELL ENGINEERS, NDT LABORATORY

ATTN: MR. D ORLOSKY

ATA WEST MAIN STREET

CT 06357

CROFT ASSOC 0700856

CROFT ASSOCIATES LIMITED

ATTN: MR TREVOR N. WALKER

82 NOR CULHAM ESTATE

OXFORDSHIRE, UK

OX14 3GY

$$
\begin{aligned}
07100404 \\
\text { SKA INC. }
\end{aligned}
$$

CTI ALASKA, INC

ATTN: MR. SANDY N. WATSON

A 31 OLD SEWARD HWY SUITE 107

ANCHORAGE AK 99503

07100301

CTL ENGINEERING INC

ATTN: MR J L. CROWLEY, RSO

2860 FISHER ROAD

COLUMBus

$\mathrm{OH} 43204$

\section{5}

DAIRYLAND POWER COOPERATIVE

ATTN: RADIATION SAFETY OFFICER

OUTE 1, BOX 275

WI 54632

DAYTON X-RAY 07100252

ATTN: MR RICHARD J. SAMMONS

705 ALBANY STREET

$\mathrm{OH} 45408$ 

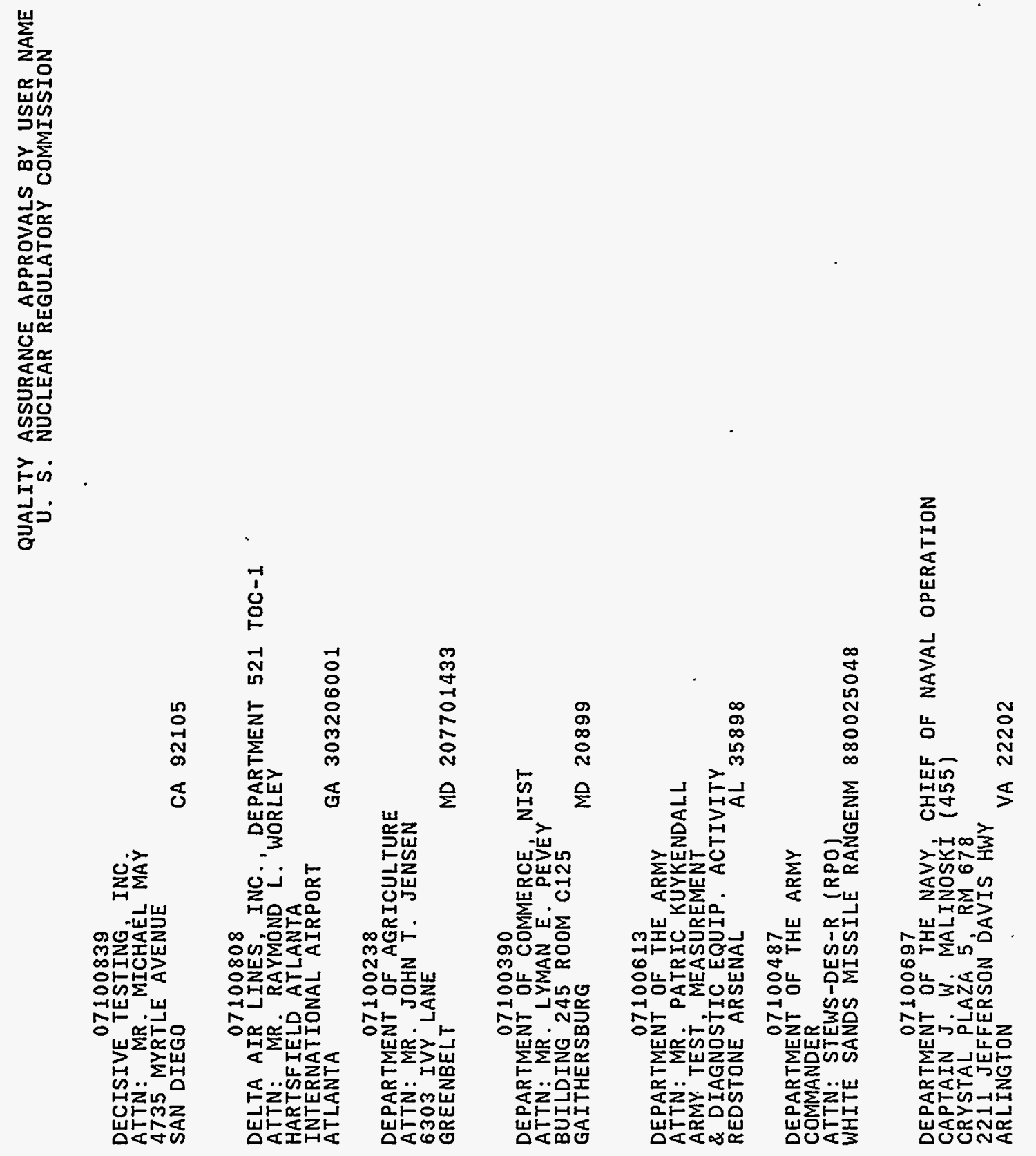


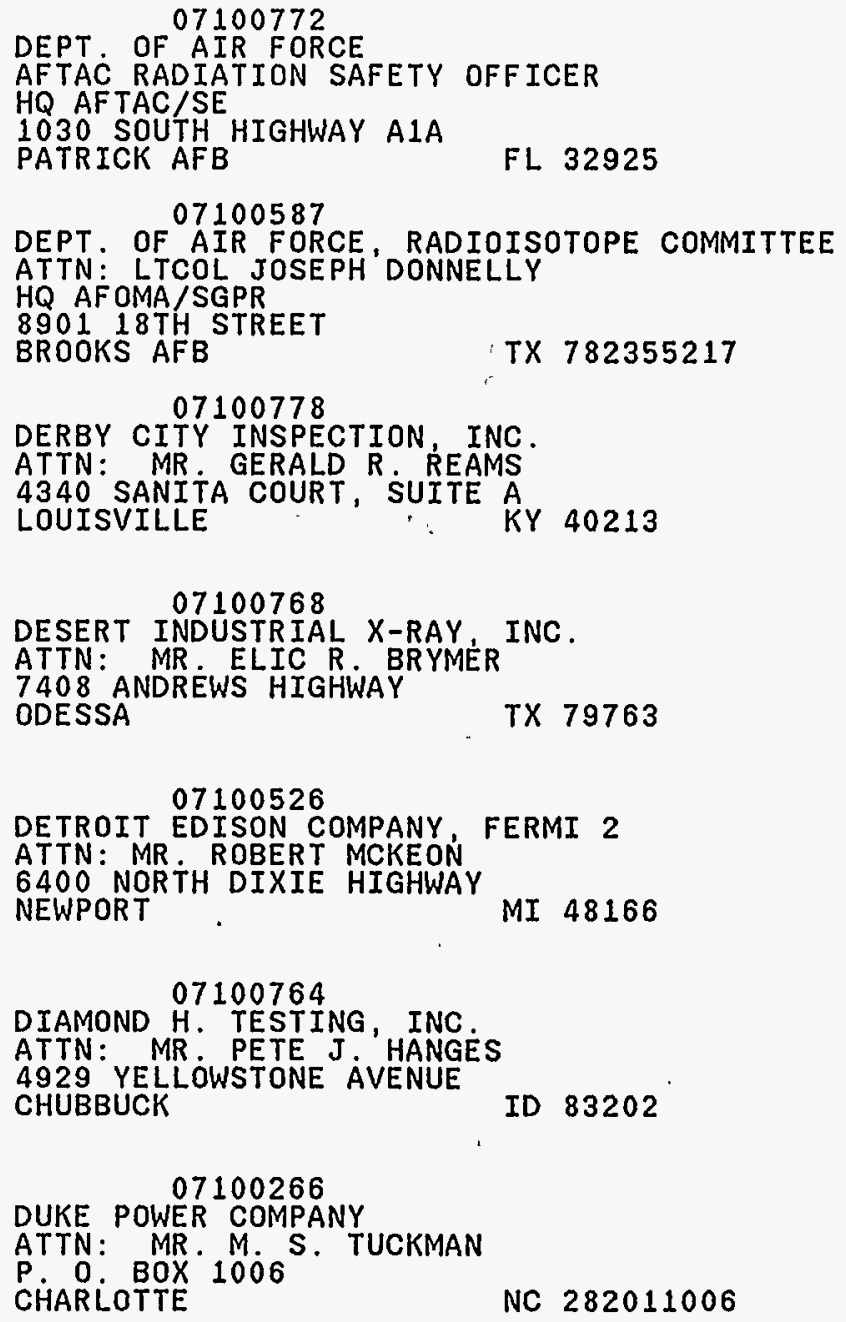

NC 282011006 


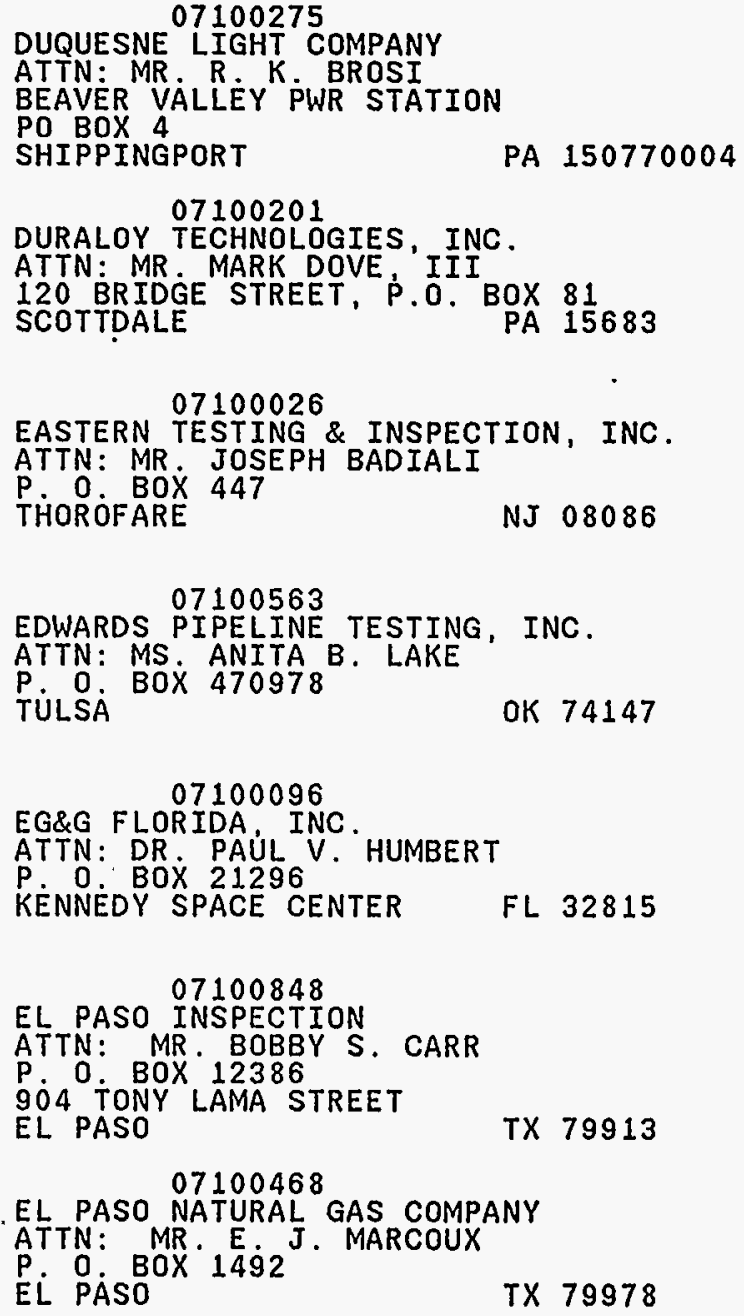




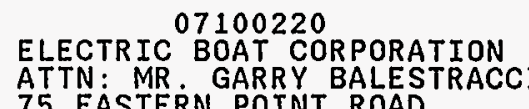

CT 063404989

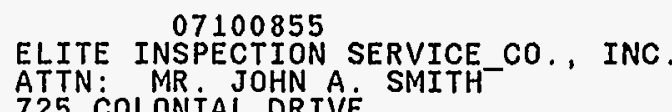

725 COLONIAL DRIVE

IN 46342

\section{8}

ENTEC CONSULTANTS, INC.

ATTN: MR ROBERT WAGNER

925 BROADWAY

NY 12207

ENTERGY OPERATIONS INC.

ATTN: MR MARK L. CARVER

P. 0 . BOX 756

MS 39150

07100604

ENTERGY OPERATIONS, INC

ATTN: MR. JAMES J. 'FISICARO

WATERFORD 3

KILLONA

LA 70066

07100341

ENTERGY OPERATIONS, INC

ATTN: MR. DWIGHT C. MIMS

RUSSELLVILLE

AR 72801

07100566

ENTERGY OPERATIONS, INC., RIVER BEND STATION

ATTN: MR. DAVID LORFING

$5 \dot{4} 85$ U.S. HIGHWAY 61

5485 U.S. HIGHWA
ST. FRANCISVILLE

LA 70775 
07100810

ENVIRONMENTAL TECHNOLOGIES, INC.

ATTN: MR ROGER L. SHINGLETON

AUBURN $29 T H$ STREET, NW WA 98001

FBF. INC. 07100185

ATTN : MR. H. STRINGF IELD

1201 HILTO

TN 37921

\section{9}

FLORIDA POWER \& LIGHT COMPANY

ATTN: MR. ROBERT ACOSTA

A. 0 . BOX 14000

FL 334080420

07100332

07100332

ATTN: MR. P. M. BEARD

AT760 W POWER LINE ST JR.

CRYSTAL RIVER LINE STREE FL 344286708

07100099

FOSTER WHEELER ENERGY CORPORATION

ATTN: MR $S$ J . AKRIN

DANSVILLE

NY 14437

07100115

ATTN: MS. GAYLE F. ELLIOTT

P.O BOX 11646

VA 245061646

07100157

FROEHLING \& ROBERTSON, INC

ATTN: MR. WILLIAM BRIODY

3015 DUMBARTON ROAD, BOX 27524

RICHMOND

VA 232617524 


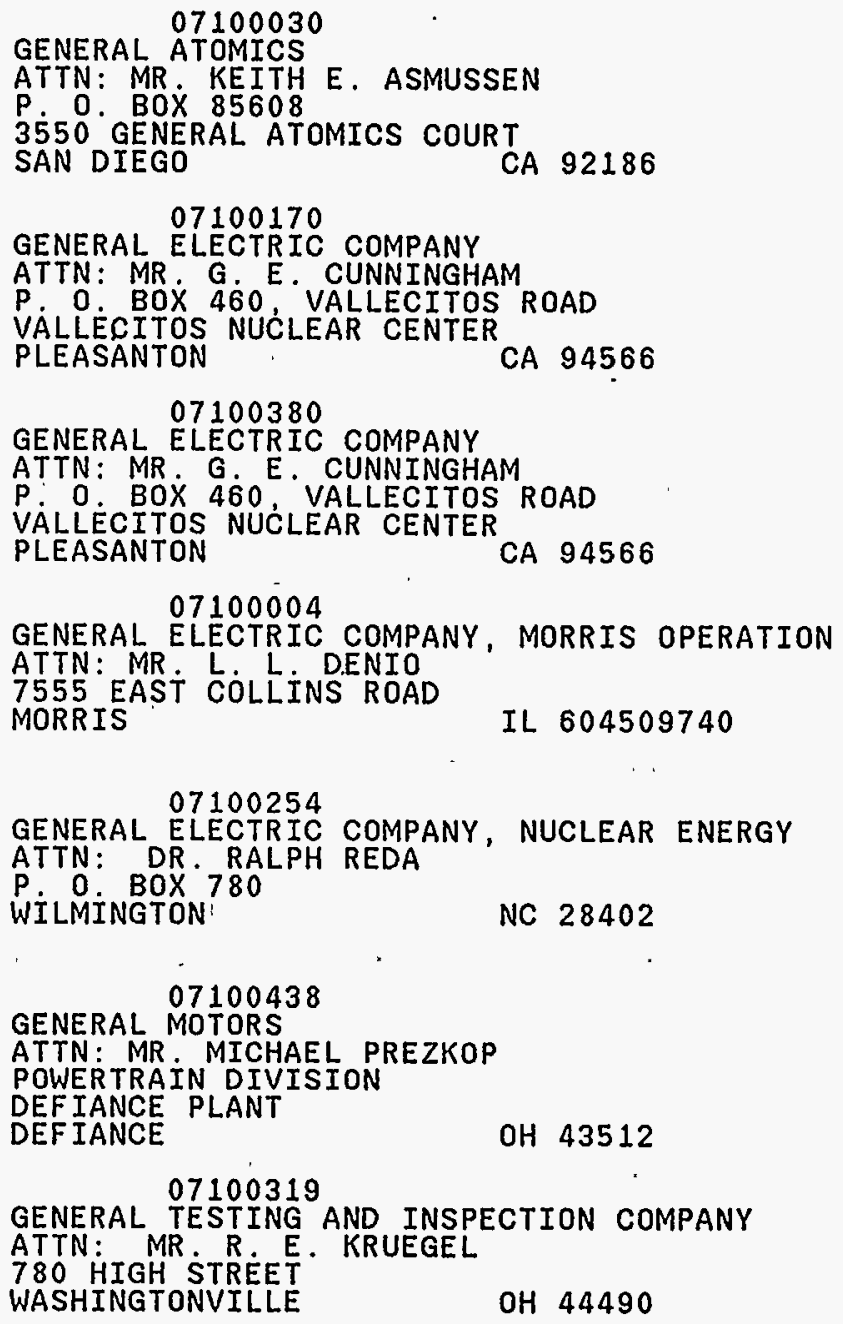


GEORGIA INSTITUTE OF TECHNOLOGY

ATTN: DR. R. A. KARAM

NEELY NUCLEAR RESEARCH CENTER

9O0 ATLANTIC DRIVE

GA 303320425

07100521

GEORGIA POWER CÓ PLANT E. I. HATCH

ATTN: MR W. G

40 INVERNESS CTR PARKWAY

BIRMINGHAM

$A L 35201$

\section{6}

GEORGIA POWER COMPANY, VOGTLE PROJECT ATTN: MR.C. K. MCCOY

40 INVERNESS CENTER PARKWAY,

BIRMINGHAM

AL 35201

07100171

GLITSCH FIELD SERVICES/NDE, INC

ATTN: MR. ALAN C. MAGNO

5250 MAYFAIR ROAD,

NORTH CANTON

$\mathrm{OH} 44720$

07100146

GLOBE $X-R A Y$ SERVICES, INC

ATTN: MR. KENNETH SWANSON

8441 SOUTH UNION

OK 74132

07100355

GPU NUCLEAR CORP. OYSTER CREEK

ATTN: MR. ROBERT HILLMAN

P. 0 BOX 388

ROUTE 9 SOUTH

NJ 170570480

07100334

GPU NUCLEAR CORPORATION, TMI

ATTN: MR . JOHN S SCHORK

$P$. O. BOX 480, ROUTE 441 SOUTH

MIDDLETOWN 
GREAT LAKES 07100769

ATTN: MRES TESTING INC

AT

GREEN BAY

WI 54304

07100200

GREDE-PRYOR, INC, $C / 0$ GREDE FOUNDRIES, INC.

ATTN: MR. JAMES O. WHITE

MILWAUKEE

WI 532260499

07100036

GRINNELL CORPORATION

ATTN: MR WILLIAM GOLINI

160 FRENCHTOWN ROAD

NORTH KINGSTOWN

RI 02852

\section{5}

H \& G INSPECTION COMPANY, INC.

ATTN: MR. H. W. GIBSON

HOUSTON

TX 77272

07100423

$H$ \& $H X$ X-RAY SERVICES, INC.

ATTN: MR. K. Y. HEAD, RSO

110 BARR LANE

WEST MONROE

LA 712940517

\section{5}

H. R. INSPECTION SERVICE

ATTN: MR. ROBERT WILLIAMSON

SHAWNEE MISSION

KS 66218

07100498

HALLIBURTON LOGGING SERVICES

ATTN: MR JEFF PETTIGREW

HOUSTONOX 42800

TX 772422800 
07100051

HARRISON STEEL CASTINGS CO

ATTN: MR. WILLIAM D. HOLLANDER

ATTICA

IN 47918

07100553

HIGH MOUNTAIN INSP SERVICES, INC.

ATTN: MR C R CLICK CLINE

1581 DERR ICK DRIVE

WY 82601

HIGH 07100186

HIGH STEEL STRUCTURES, INC.

A 0 : BOX 10008 LONG

LANCÁSTER

PA 17605

07100784

HOLTEC INTERNATIONAL

ATTN: MR MARK SOLER

NJ 080031666

07100317

ATTN. MR LOR

P O BOX 210 .

BÉASLEY

TX 77417

07100093

ATTN: MR TESTING INC

PTTN: MR. CLYDE W. MAY

HUNTINGTON

WV 25717

\section{7}

ICF KAISER HANFORD COMPANY

ATTN: MR WILLIAM S. ROE

RICHIAND 888

WA 99352 
IES UTILITIES 071006

IES UTILITIES INC, FRANZ, JR.

P. BOX 35

CEDAR RAPIDS

IA 52406

\section{4}

ILLINOIS POWER COMPANY

ATTN: MR. MICHAEL W. LYON

CLINTON PWR STA. P.O. BOX 678

CLINTON

07100775

IMPERIAL INSPECTION INC

ATTN: MR. JERRY WAGGONER

13 ROYAL STREET

WEST MONROE

LA 71291

INDEPENDENT TESTIN

INDEPENDENT TESTING LABORATORIES

ATTN: MR WALTER C. CRAGER

19424 PARK ROW, NO. 110

HOUSTON

TX 77084

07100377
INDIANA MICHIGAN POWER COMPANY

ATTN: MR E.E; FITZPATRICK

$\mathrm{OH} 43216$

07100840

INDUSTRIAL MARINE TESTING LABORATORY I 7 : MMRE SAMUEL A ${ }^{\circ}$ BOYKIN

ATI

CA 921131426

07100326

INDUSTRIAL NDT SERVICES DIVISION

ATTN: MR. JAMES M. THOMPSON

2124 WENDELL AVENUE

$P$. BOX 2245

IN 46202 
QUALITY ASSURANCE APPROVALS BY USER NAME

$U$. S. NUCLEAR REGULATORY COMMISSION

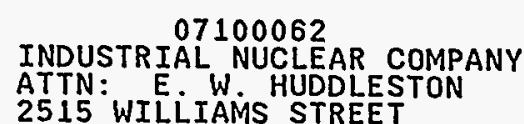

SAN LEANDRO

CA 94577

07100119

INGERSOLL-RAND CO.

ATTN: MR MATTHEW M. MORGANELL

PHILLIPSBURG

NJ 08865

\section{7}

INSPECTION MANAGEMENT CORPORATION

ATTN: MR D $\dot{1}$ L MYERS

BIXBY OK 74008

07100863

INSPECTION SERVICE, INC

ATTN: MR TONY M. MARTIN

KENNEWICK

07100213

INTERMOUNTAIN TESTING CO

2965 SOUTH SHOSHONE STREET

ENGLEWOOD. SHOSHONE STREET 80110

\section{2}

INTL. RADIOGRAPHY \& INSP. SERV.

ATTN: MR JOHN T CHARTER

$7620 W$. MELROSE LANE

OK 76302

07100813

IOWA STATE UNIVERSITY OF SCIENCE AND TECHNOLOGY

ATTN: MR SCOTT WENDT

107 NUCLEAR ENGINEERING LAB

AMES IA 500112241 
ITT INSPECTION 07100830 TECH TECHNICIANS, INC.

ATTN: MR. $C . V$. ROUDEBUSH

KÄNÁS CITY

MO 64106

J. L. SHE7100122

J SHEPHERD \& ASSOCTATES

1010 ARROYO ST.

SHEPHERD

SAN FERNANDO

CA 91340

JAN X-RAY CO.

ATTN: MR J IA. NEWLAND

PARMA

MI 49269

KC INSPECTION INC

ATTN: KELLEYD. GASTON

VENTURA

CA 93005

KOONEY X 07100761

KOONEY X-RAY, INC
ATTN: MR. S: F. FRAZIER

ATTN: MR. S.

BÄRKËR

TX 77413

07100787

07100787 SERVICES, INC

LAFAYETTE TESTING SERVICES

P : D. BOX 6682

IN 47903

07100853

AUREL TECHNOLOGIES, INC.

ATTN: MS VICTORIA A. BORLAND

LAUREL

MD 20707 


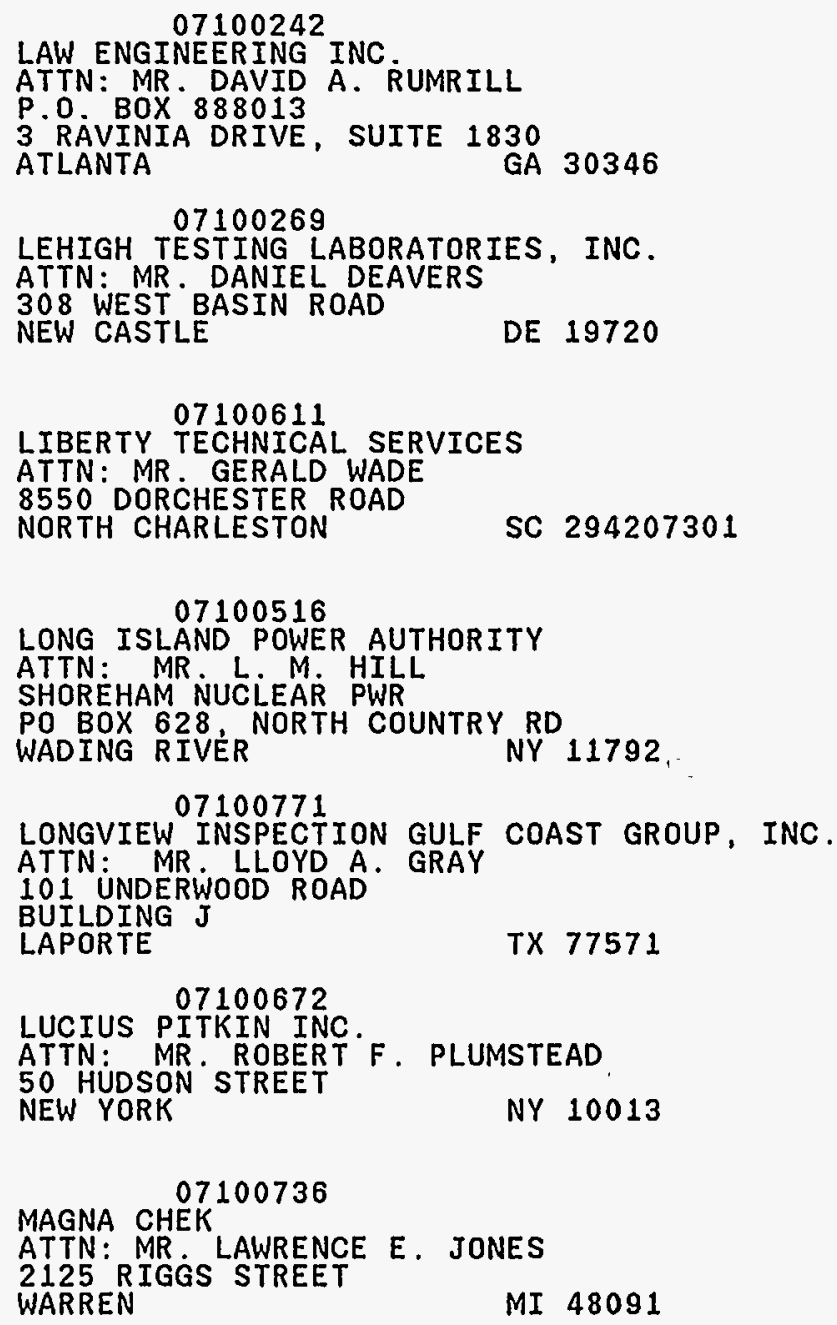

LONG ISL 07100516

LONG ISLAND POWER AUTHORITY

ATTN: MR $L L$ M. HILL

PO BOX 628 NORTH COUNTRY RD

PO BOX 628
WADING RIVER NORTH COUNTRY RD
NY 11792

07100771

LONGVIEW INSPECTION GULF COAST GROUP, INC

ATTN: MR L LLY A. GRAY

ATTN: MR. LLOYDA.

BUILDING

TX 77571

07100672

LUCIUS PITKIN INC. $F$. PLUMSTEAD

ATTN: MR ROBERT F. PLUMSTEAD.

NEW YORK NY 10013

MAGNA CHEK

07100736

ATTN: MR. LAWRENCE E. JONES

2125 RIGGS STREET

WARREN

MI 48091 
MAINE YANKEE ATOMIC POWER CO.

ATTN: MR. JAMES R. HEBERT

329 BATH ROAD

ME 04011

MANOIR EITETROAL

MANOIR ELECTROALLOYS CORPORATION

ATTN: MR DAVID MCINTIRE

TAYLOR STREET AND ABBE ROAD

P.O. BOX 401

$\mathrm{OH} 440362012$

07100807

MARYLAND Q. C. LABORATORIES, INC

ATTN: MR BRUCE E. DEWEY

$P . O B O X$

MD 21017

07100316

MASON \& HANGER-SILAS MASON CO., INC

ATR. MR. E. SHANNON

MIDDLETOWN

07100164

MASSACHUSETTS INSTITUTE OF TECHNOLOGY

ATTN: MR THOMAS H. NEWTON, JR

CAMBR IDGE

MA 02139

07100111

MATERIAL TESTING LABORATORIES, INC.

ATTN: MR JAMES R. CANNON

1531 EARLY STREET

NORFOLK

VA 235021603

07100542

MATTINGLY TESTING SERVICES, INC.

ATTN: MR . MARK M. MATTINGLY

P. $O$ BOX 3126

MT 59403 


\section{MAXIM TECHNOT103}

ATTN: MR NALVGE, INC.

662 CROMWELL AVE.

ST. PAUL

MN 551141776

MAXTM TEC 07100712

MAXIM TECHNOLOGIES, INC. /EMPIRE SOILS INVEST.

ATT: BOX PAUL KOWELL, RSO

PAं

07100801

MCNDT LEASING INC

ATTN: MR JIM MCCAIN

CHANNAHON

IL 60410

07100817

MESCALERO ENVIRONMENTAL, INC

ATTN: MR. FRED PESO

P. O BOX 176

MÉSCÁLERO

NM 88340

\section{9}

MET-CHEM TESTING LABS

ATTN: MR . N. WAYNE HANSEN

369 WEST GREG WON AVE.

SALT LAKE CITY

UT 841153440

\section{4}

METALS EVALUATION AND TESTING, INC.

ATTN: MR BROOK MASSEY

35 HEGENBERGER PLACE

CA 94621

07100851

MICHIGAN TECHNOLOGICAL UNIV

ATTN: MR DONALD A. DAAVETTILA

1400 TOWNSEND DRIVE

HOUGHTON

MI 499311295 
07100694

MID AMER ICAN INSPECTION SERVICES, INC

ATTN: MR. TERRY L. WILKINS

MIE ROAD, P.O. BOX 1427

GAYLORD

MI 49735

\section{0}

MIDLAND INSPECTION AND ENGINEERING, INC.

ATTN: MR RICHARD C . HAGER III

14214 WEST US HIGHWAY 80 EAST
ODESSA

\section{4}

MIDWEST INDUSTRIAL X-RAY INC.

ATTN: MR. MICHAEL G. MANGER

PIO2. 7 TH AVE. NORTH

FARGO

ND 58107

07100476

MIDWEST INSPECTION SERVICES, ING.

ATTN: MR RAYMOND EZZELL

514 SOUTH MAIN, P.O. BOX 1229

22979070

MINNESOTA VALLEY ENGINEERING INC.

ATTN: MR RICK MINETTE

P. $\mathrm{BOX} 234$

MN 56071

07100408

MISSOURT STEEL CASTINGS COMPANY

ATTN: MR DAVID GUTHRIE

P. $B 0 \mathrm{X}$ i706

JOPLIN

MO 64802

07100753

MOBILE-LAB, INC

ATTN: MR. HERBERT SIMPSON

P. 00 BOX 395

LA 70059 
MONTANA 07100494

MONTANA X-RAY INC

P.O. BOX 50 i 33

MT 59105

07100585

MQS INSPECTION INC

ATTN: MR E. L. BAC

ELK GROVE VILLAGE

IL 60007

07100331

N.C. STATE UNIVERSITY

AT : MR. MERALD WICGS

A $B 0 X 7909$

RÁLEIGH

NC 27695

07100018

NAC INTERNATIONAL, INC.

ATN: MR GARY TJERSLAND

655 ENGINEER ING DRIVE

SUITE 200

NAC INTERNATIONAL. INC.

GA 30092

ATN M JOHN J STOBBS

655 ENGINEERING DRIVE

NORCROSS

GA 30092

07100444

NATIONAL INSPECTION \& CONSULTANTS, INC.

ATTN: MR G. VAUGHAN LAKE

AOT EVANS AVE

LAND MARK BUILDING, STE 207

FORT MEYERS

FL 33901

07100759

NATIONWIDE TESTING SERVICES, INC.

ATTN: MR. DAVID H: SIMON

B11 MORSE AVENUE

SCHAUMBURG

IL 60193 
NDT SERVICES INC.

ATTN: MR. RÓDNEY LILLARD

CAGUAS ${ }^{B O X} 4952$, SUITE 370 PR 007264952

NDT SPECIAL 07100701

ATTN: MR. L. J INCHNEIDER JR

1739: SOUTH CARFERRY DRIVE

MILWAUKEE CARFERRY DRIVE WI 53207

\section{4}

DISTRICT

ATTN: MR. J. H. MUELLER

BROWNVILLE

NE 68321

07100711

NEN LIFE SCIENCES COMPANY

ATTN: MR. LEONARD R. SMITH

549 ALBANY STREET

MA 02118

NEUTRON 07100121

ATTN: MR. FRANK SCHWOERER

22301 MT. EPHRAIM ROAD

DICKERSON

MD 20842

\section{NEW YORK 07100240 \\ YORK POWER AUTHORITY}

ATTN: MR. W. A. JOSIGER

WHITE PLAINSET

07100240

NY 10601

NEW YORK POWER AUTHORITY, INDIAN POINT UNIT. \# 3

ATTN: MR PAUL SAUNDERS

BÜCHANAN

NY 10511 

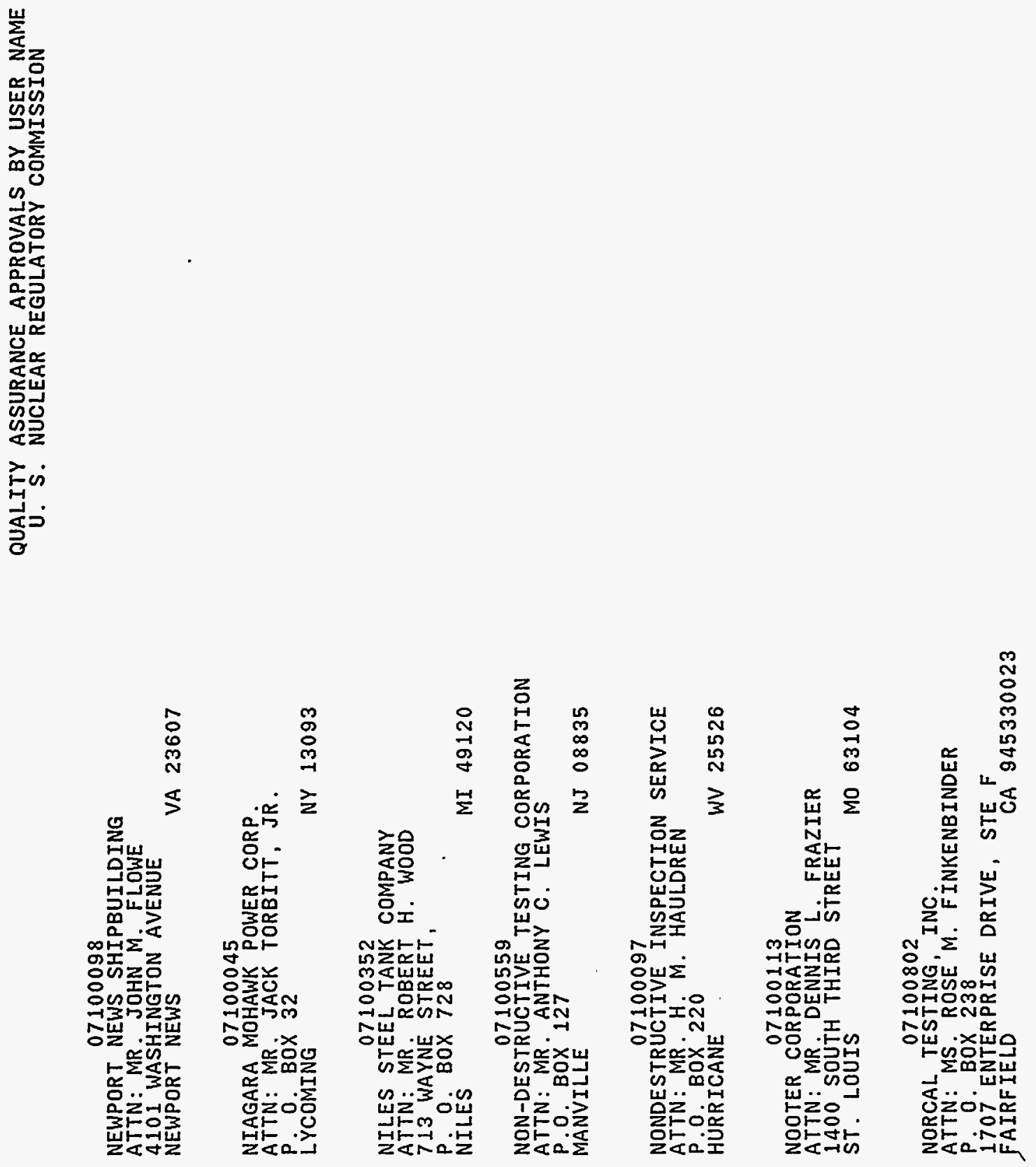


\section{3}

NORDION INTERNATIONAL INC.

ATTN: MR. P. A. GRA

KANATA, CANADA

$K 2 K 1 \times 8$

07100075

NORFOLK SHIPBUILDING \& DRYDOCK COMPANY

ATTN: MR. CARL A. CHERRY

NORF́OLK

VA 235012100

07100514

NORTH AMERICAN INSPECTION, INC.

5260 WEST

WHITEHALL

PA 18052

07100835

NORTH ATLANTIC ENERGY SERVICE CORPORATION

ATTN: MR. TED C FEIGENBAUM

P. O. BOX 300

SEABRंOOK

NH 03874

07100207

NORTHEAST NUCLEAR ENERGY COMPANY

ATTN: MR. J.F. OPEKA

P. O. BOX 270

CT 061410270

07100083

NORTHERN STATES POWER CO.

ATTN: MR : MARVIN ENGEN

MONTICELLO

07100016

NORTHWEST AIRLINES

ATTN: MR J JEFF A. REGISTER

5101 NORTHWEST DRIVE

M/S C 8840

MN 551113034 
NORTHWEST INSPECTION AND TESTING SERVICES, INC.

ATTN: MR. WILLIAM H. SHAMEL

IDAHO FALLS

ID 83405

NOVA DATA 07100628

ATTN. MR JOHN TAYLABS

204 NORTH MAIN STREET, \#201

HOPEWELL WAIN STREET, "VÁ 23860

\section{9}

NUCLEAR CONTAINERS, INC

ATTN: MR. WILLIAM M. ARNOLD

125 IODENT WAY, SUITE

ELIZABBETHTON

TN 37643

NUCLEAR 07100249

作

ATTN: MR. ANDREW M. MAXIN

ERWIN

TN 37650

07100825

OCEAN STATE TECHNICAL SERVICES

ATTN: MR. JAMES J. GIARRUSSO

2440 MENDON ROAD

CUMBERLAND

RI 028643799

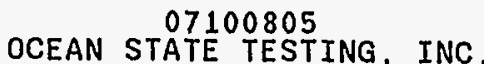

ATTN: MR. FRANK L. MCCANN

TREE

RI 02852

07100770

OHM REMEDIATION SERVICES CORPORATION

ATTN: MS ROBERTA SUMMERS

250 BERRYHILL ROAD, SUITE 400

COLUMBIA

SC 29210 
QUALITY ASSURANCE APPROVALS BY USER NAME

$U$. S. NUCLEAR REGULATORY COMMISSION

OMAHA PUB100256

OMAHA PUBLIC POWER DISTRICT

ATTN: MR . W. G. GATES

444 SOUTH 16 TH STREET MALL
OMAHA

07100202

PACIFIC GAS \& ELECTRIC CO.

ATTN: MR . GREGORY $M$. RUEGER

SAN FRANCISCO

07100773

PACIFIC RIM CONSULTING AND INSPECTION CORP

ATTN: MR TERRY R. TAMBERG

375 KOAPAKA STREET NO. H-465

HONOLULU

$H-465$
$H T$ g 6819

07100682

PACIFIC TECHNICAL INDUSTRIES, INC.

ATTN: MR. DOUGLAS L. NALLEY

1414127 TH PLACE, N.E.

NELIEVUE

WA 98005

07100838

PACIFIC TESTING LABORATORIES

ATTN: MR EMERYE. ROBERTS

3257 16TH AVENUE WEST

SEATTLE

WA 98119

07100678

PACKAGING TECHNOLOGY, INC

ATTN: MR. RICHARD T' HAELSIG

4507-D PACIFIC HIGHWAY EAST

TACOMA

07100842

PANHANDLE NDT AND INSPECTION

ATTN: MR. ORVIL COUCH

P. ODOX 1474

BÖRGER

TX 79008 
07100729

PAUL SINN TESTING SERVICES

ATTN: MR. PAUL W. SINN

MOX 185

IL 62257

07100056
PECO ENERGY COMPANY

ATTN: MR. G. A: HUNGER, JR.

965 CHESTERBRंOOK BLVD.

PA 190875691

07100525

PECO ENERGY COMPANY, NUCLEAR GROUP HEADQUARTERS

ATTN: MR G A A HUNGER

WAYNE

WAYNE

PA 190875691

07100471

PENNSYLVANIA POWER AND LIGHT COMPANY

ATT

ALLENTOWN PA 181011179

07100470

PENNSYLVANIA STATE UNIVERSITY

ATTN: MR . DAVID A. SHIRLEY

UNIVERSITY PARK

PA 168021503

07100821

PENSACOLA TESTING LABORATORIES, INC

ATTN: MR . PATRICK A. WHEELER

217 EAST BRENT LANE

FL 32503

\section{1}

PERRY NUCLEAR POWER PLANT

MGR . REGULATORY AFFAIRS SECT.

10 CENTER ROAD

OH 44081 
QUALITY ASSURANCE APPROVALS BY USER NAME
U. S. NUCLEAR REGULATORY COMMISSION

07100667

PITT-DES MOINES INC

ATTN: MR. J.F STRUNK

PITTSBURGH

PA 15225

07100327

PORTLAND GENERAL ELECTRIC CO. TROJAN PLANT ATTN: MR STEPHEN M. QUENNOZ

PORTLAND

07100221

PRECISION COMPONENTS CORP

ATTN: MR. FRANCIS G. KARCHNAK

P. BOX 15101

PA 17405

07100829

PRIVATE FUEL STORAGE LIMITED LIABILITY COMPANY

ATTN: MR. JOHN D. PARKYN

P.O BOX C4010

WI 546024010

07100781
PROFESSIONAL QUALITY TESTING COMPANY

ATTN: MR. JOHN C. CHABAL

NEW KENSINGTON

PA 15068

07100440

PROFESSIONAL SERVICE INDUSTRIES, INC

ATTN: MR DAVID S PRICE

LOMBARD

IL 60148

07100656

PROFESSIONAL WELDING ASSOCIATES, INC.

ATTN: MR GARY MARQUARDT

E3349 CITY HIGHWAY

WI 54216 
07100630
PROGRESS SERVICES, INC.
ATTN: MS M. BECKER
4170 COLUMBIA ROAD

MEDINA

OH 44256

PSI ENERGY 0700640

ATTN: MR - GENE DAVIS

1000 EAST MAIN STREET

PLAINF IELD

IN 46168

\section{6}

PUBLIC SERVICE COMPANY OF COLORADO

ATTN: MR FRERICK J. BORST

16805 WELD COUNTY ROAD $19-1 / 2$
PLATTEVILLE

07100328

PUBLIC SERVICE ELECTRIC AND GAS COMPANY

ATTN: MR RUSSELL AVESON

HANCOBX 236

NJ 08038

07100577
PURDUE UNIVERSITY

ATTN: MR. JAMES F. SCHWEITZER

CIVIL ENGINEER ING BUILDING

WEST LAFAYETTE

IN 47907

OC LABORAT100429

QC LABORATORIES, INC.

ATTN: MR R D.' STICKLER

ADLLYTIRLING ROAD

FL 330201199

QSL INSPECTION 07100721

ATTN: MR MICHAEL J. LANGE

7 .

PA 19006 
QUALITY ASSURANCE APPROVALS BY USER NAME

$U$. S. NUCLEAR REGULATORY COMMISSION

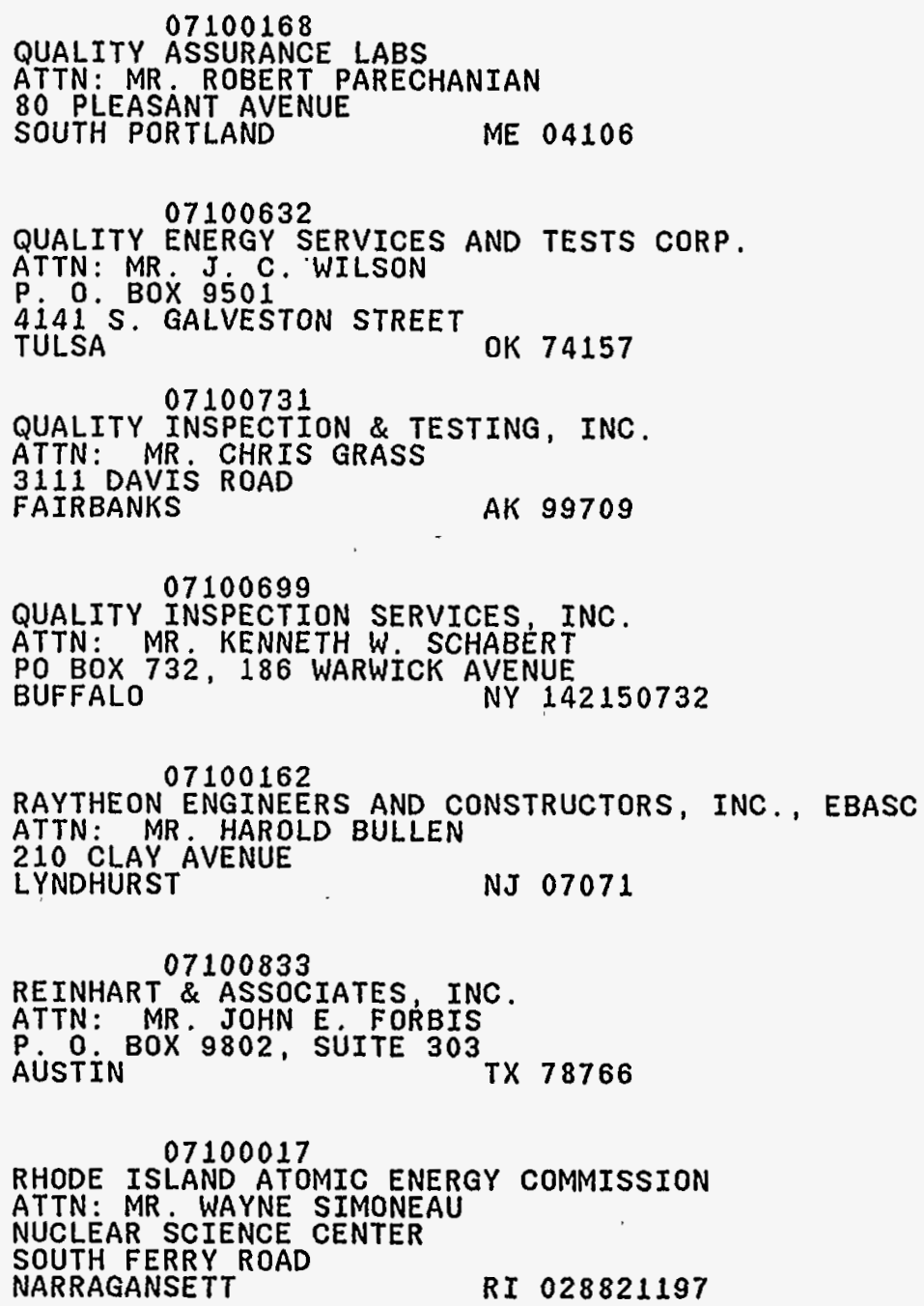

AK 99709

07100699

QUALITY INSPECTION SERVICES, INC

ATTN: MR. KENNETH W. SCHABERT

PO BOX 732, 186 WARWICK AVENU

BUFFALO

NY 142150732

\section{2}

RAYTHEON ENGINEERS AND CONSTRUCTORS, INC., EBASC ATTN: MR HAROLD BULLEN

AYT CLAY AVENUE

NJ 07071$$
07100833
$$

REINHART \& ASSOCIATES, INC.

ATTN: MR. JOHN E. FORBIS

AUSTIN $30 X$ 9802, SUITE 303 TX 7876

07100017

RHODE ISLAND ATOMIC ENERGY COMMISSION

ATTN: MR. WAYNE SIMONEAU

NUCLEAR SCIENCE CENTER

SOUTH FERRY ROAD

NARRAGANSETT

RI 028821197 


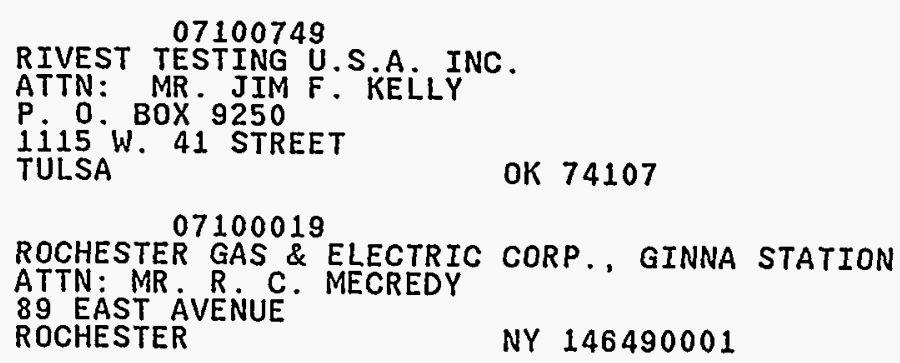


07100496

SCIENTIFIC ECOLOGY GROUP, INC.

ATTN: MR J F MCCARTER

1560 BEAR CREEK ROAD,

OAK RIDGE

TN 37831

07100501

SCIENTIFIC INSPECTION TECHNOLOGY, INC

ATTN: MR DAVID $W$. IVEY

A BOX 385

TN 37343

07100609

SCIENTIFIC MEASUREMENT SYS. IN

ATTN: MR. G. A. MEYER

AUSTIN

TX 78758

07100629

SCIENTIFIC TECHNICAL, INC

ATTN: MR. BOBBY L. DENNIS

$804 F$ INDUSTRIAL AVE.

CHESAPEAKE

VA 23324

SENIOR E7100579

SENIOR ENGINEERING COMPANY

ATTN: MR GARY L. STILES

2702 W. 9 TH ST

MO 64801

07100641

SGS INDUSTRIAL SERVICES

ATTN: MR : TOM $W$. CUTHBERTSON

2506 DAVIS STREET

SAN LEANDRO

CA 94577

SHAFER VALI100600

SHAFER VALVE COMPANY

ATTN:: MR $:$ CRAIG MCCOMBS

2500 PARK AVE. WEST

MANSF IELD

OH 44906 


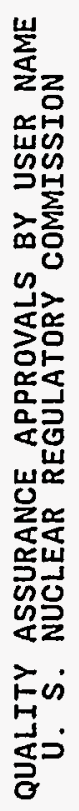

$\vec{\Xi}$

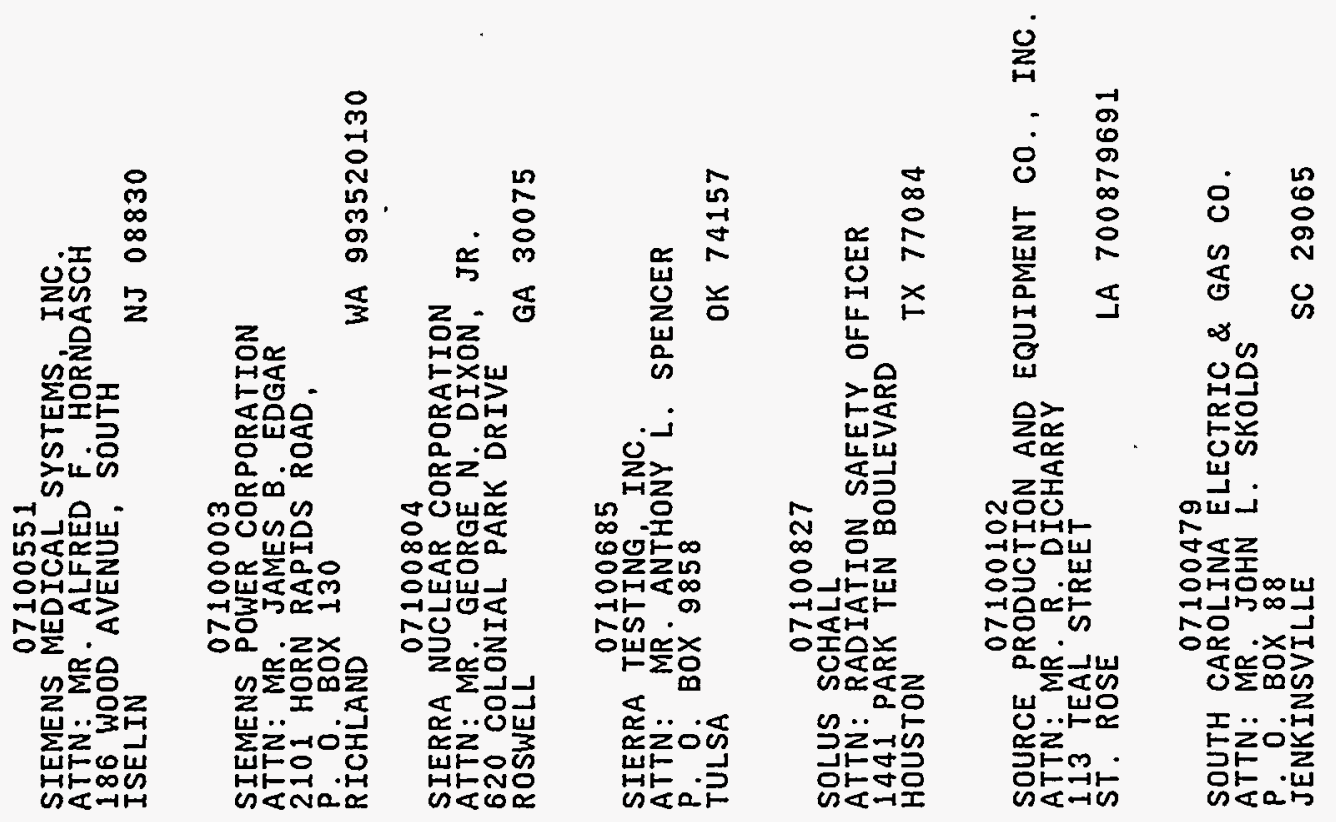


07100174

EOISON COMPANY

ATTN: MR. WALTER C. MARSH

IRVINE

CA 92718

07100333

SOUTHERN NUCLEAR OPERATING COMPANY, FARLEY PROJE

ATTN: LICENSING MANAGER

P. 0. BOX 1295

BIRMINGHAM

AL 35201

07100495

SOUTHWEST X-RAY CORPORATION

ATTN: MR. MICHAEL MCINTIRE

ROCK SPRINGS

WY 82902

07100215

SPACE SCIENCE SERVICES, INC.

ATTN: MS . NINA WALKER

ROUTE 6 BOX 210

AL 36303

07100612

SPEC CONSULTANTS INC

ATTN: MR . LOUIS C. HASS

510 PLEASANT VALLEY RD

TRAFFORD

PA 15085

07100222

ST. LOUIS TESTING LABORATORIES, INC.

ATTN: MR ROBIN SINN

2810 CLARK AVE

ST. LOUIS

MO 63103

07100226

STATE UNIVERSITY OF NEW YORK AT BUFFALO

ATTN: MR DANIEL W. SULLIVAN

ROTARY ROAD

S435 MAIN STREET

BUFFALO

NY 14214 


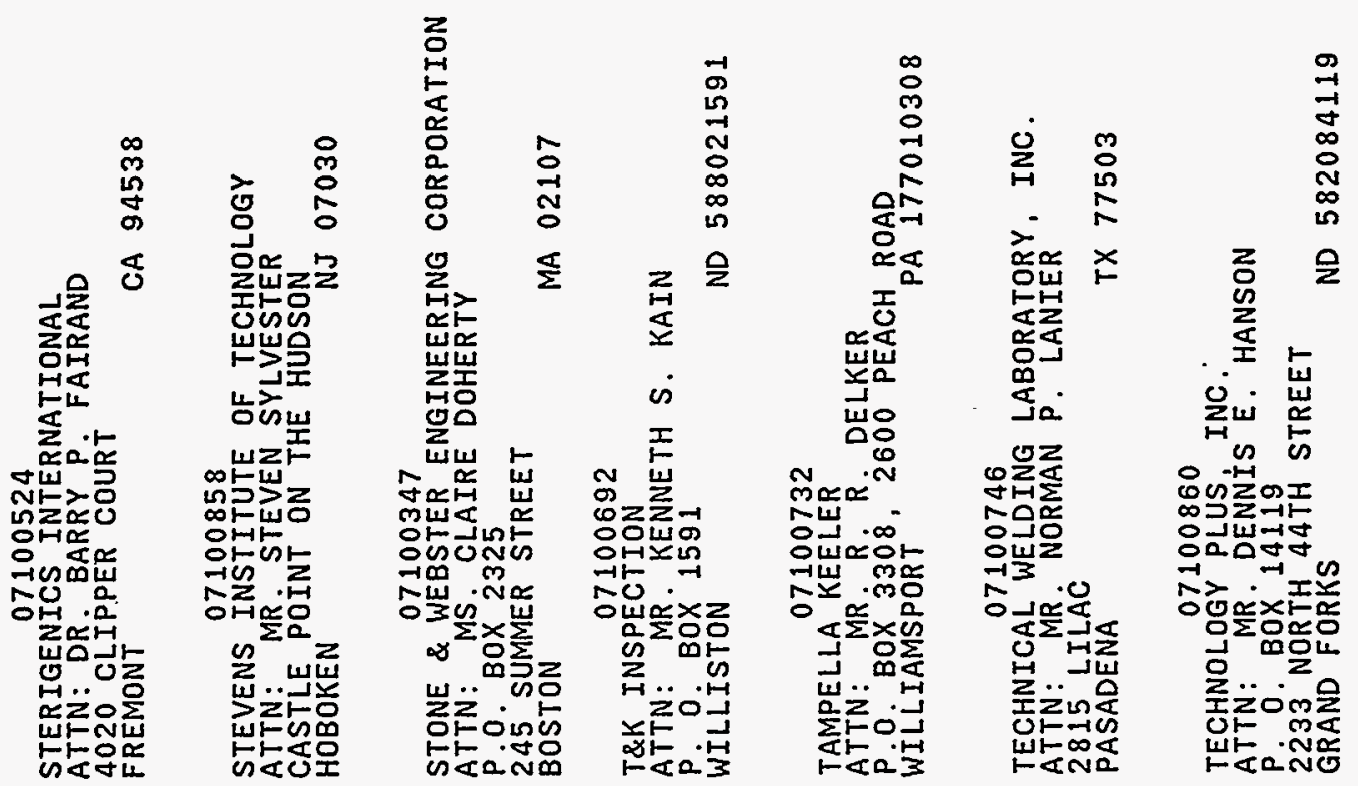


07100691

TEI ANALYTTCAL SERVICES, INC.

ATTN: MR. G. E. WEI

3. WEST

PA 15301

07100023

TENNESSEE GAS PIPELINE COMPANY

ATTN: MR. DAVID $L$. CULBERTSON

5510 SOUTH RICE AVE.

TX 77081

\section{7}

TENNESSEE VALLEY AUTHORITY

ATTN: MR. RAUL R. BARON

1101 MARKËT STREET

CHATTANOOGA

TN 374022801

07100131

TESTING INSTITUTE OF ALASKA, INC

ATTN: MR DONALD M. LOCKMAN

2114 RAILROAD AVE.

ANCHORAGE

AK 99501

TESTING 07100843

ATTN: MR R ICES \& INSPECTION, INC.

3030 MAIN STREET

SAN DIEGO

CA 92113

\section{3}

TESTING TECHNOLOGIES, INC.

ATTN: MS. JUDY CASE

WOODBR IDGE

VA 22191

\section{9}

TESTMASTER INSPECTION COMPANY

ATTN: MR. W. B. CARR

P. O BOX 31

$\mathrm{OH} 43551$ 


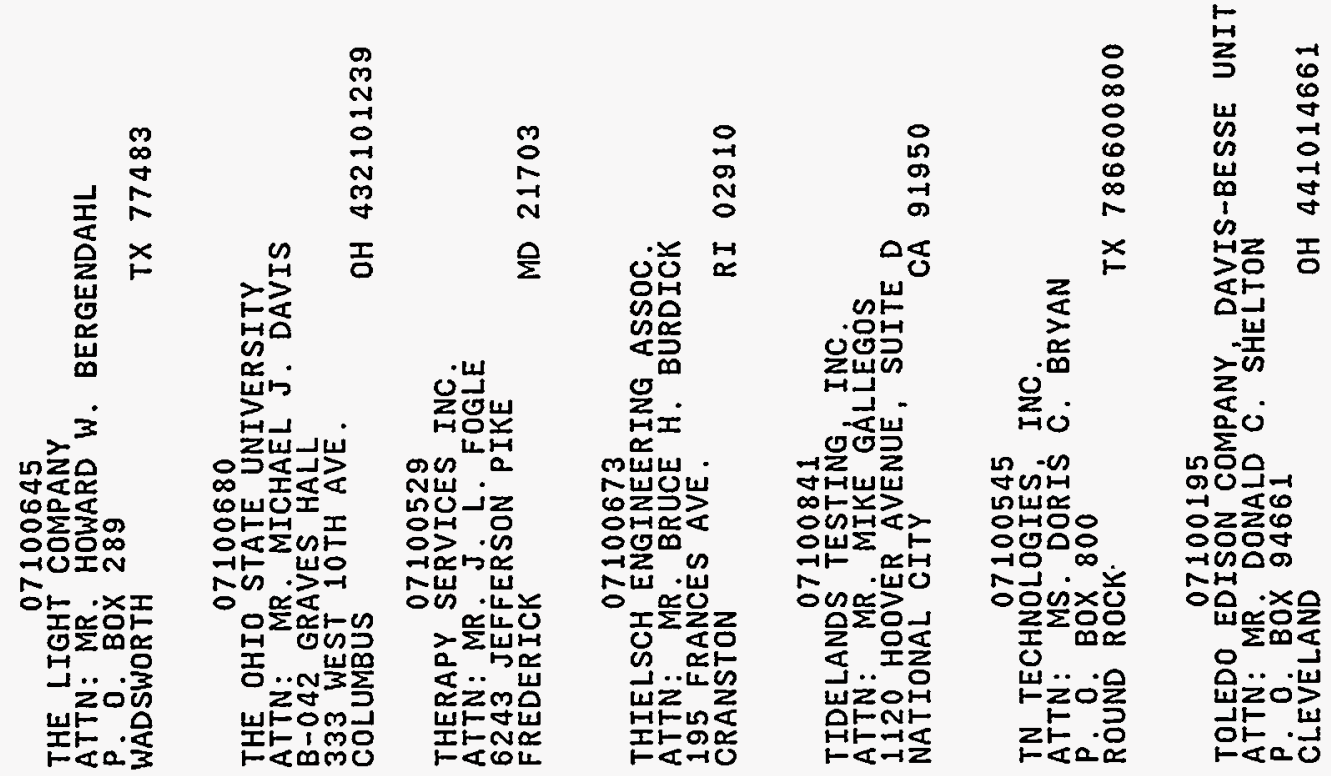




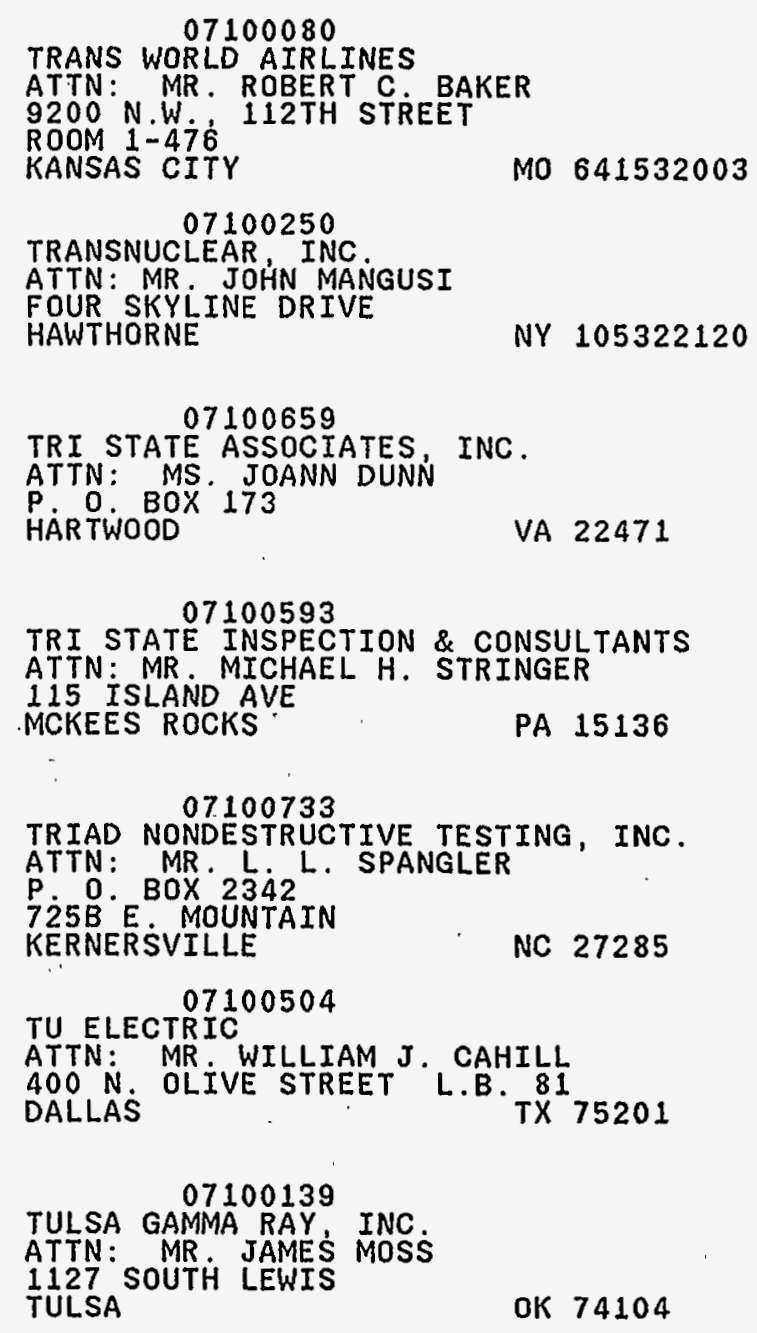




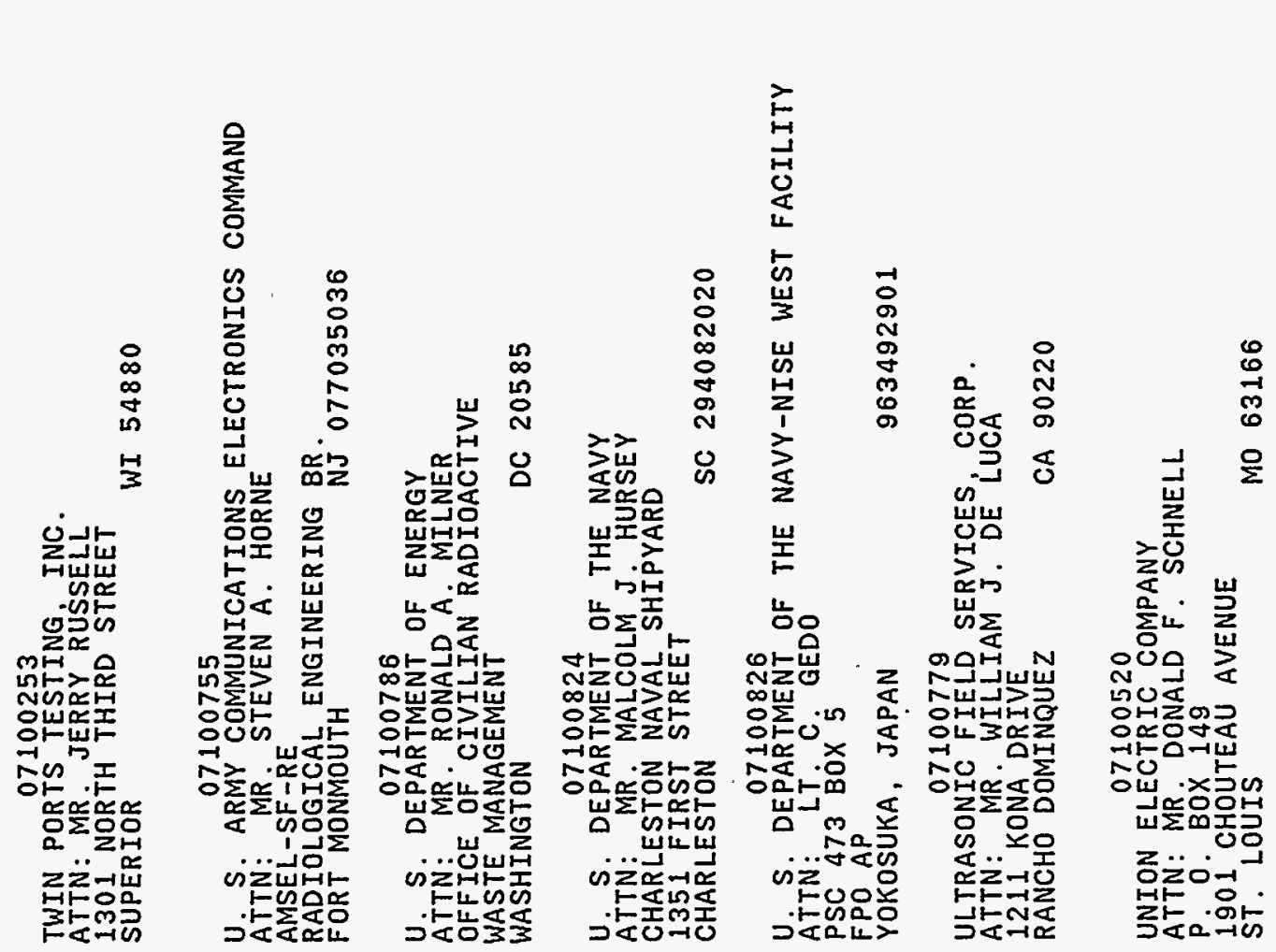




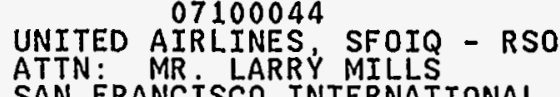

07100832

UNITED STATES ENRICHMENT CORPORATION

ATTN: MS BETH DARROUGH

6903 ROCKLEDGE DRIVE

2 DEMOCRAC

MD 20817

07100578

UNIVERSITY OF FLORIDA, NUCLEAR REACTOR FACILITY

ATTN: MR. W.G. VERNETSON

NUCLEAR REACTOR BUILDING
GAINESVILLE

FL 32611

07100482
UNIVERSITY OF MASSCHUSETTS
ATTN: MR

N414 MORRILL SCIENCE CENTER

AMHERST

MA 01003

UNTVERSTT100348

UNIVERSITY OF MICHIGAN, FORD NUCLEAR REACTOR

ATN: MR RONALD F FLMING

2301 BONTSTEEL BLVD.

07100834

MI 481092100

UNIVERSITY OF MINNESOTA

ATTN: MR JEROME W. STAIGER

$410 \mathrm{CHURCH}$ STREET, $\dot{S}$

MINNEAPOLIS

MN 55455

OF MISSOURI

A7N: MR HATEM KHOUAJA

1870 MINER CIRCLE

MO 654090630 


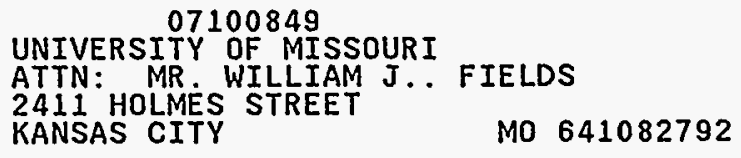

07100108

UNIVERSITY OF MISSOURI-COLUMBIA

ATTN: MR. WALT A. MEYER, JR.

RESEARCH REACTOR FACILITY

COLUMBIA

07100859

UNIVERSITY OF TULSA

ATTN: DR ALLEN R SOLTOW

DEPARTMENT OFLENYRTICS

TULSA

OK 741043189

\section{0}

UNIVERSITY OF VIRGINIA, NUCLEAR REACTOR FACILITY

ATTN: MR J J.P. FARRAR

ENG INEERING

CHARLOTTESVILLE

VA 229032442

VA POLY 07100554

VA POLY. INSTITUTE AND STATE UNIVERSITY

ATANATION DOUGLAS

BLACKSBURG

07100777

VALLEY INDUSTRIAL X-RAY AND INSPECTION SERVICES

ATTN: MR LARRY WILLIAMS

BAKERSFIELD

CA 93308

07100724

INSPECTION SERVICE, INC.

ATTN: MR. ROBERT W. KRATZER

$P$ BOX 3245

PA 18042 


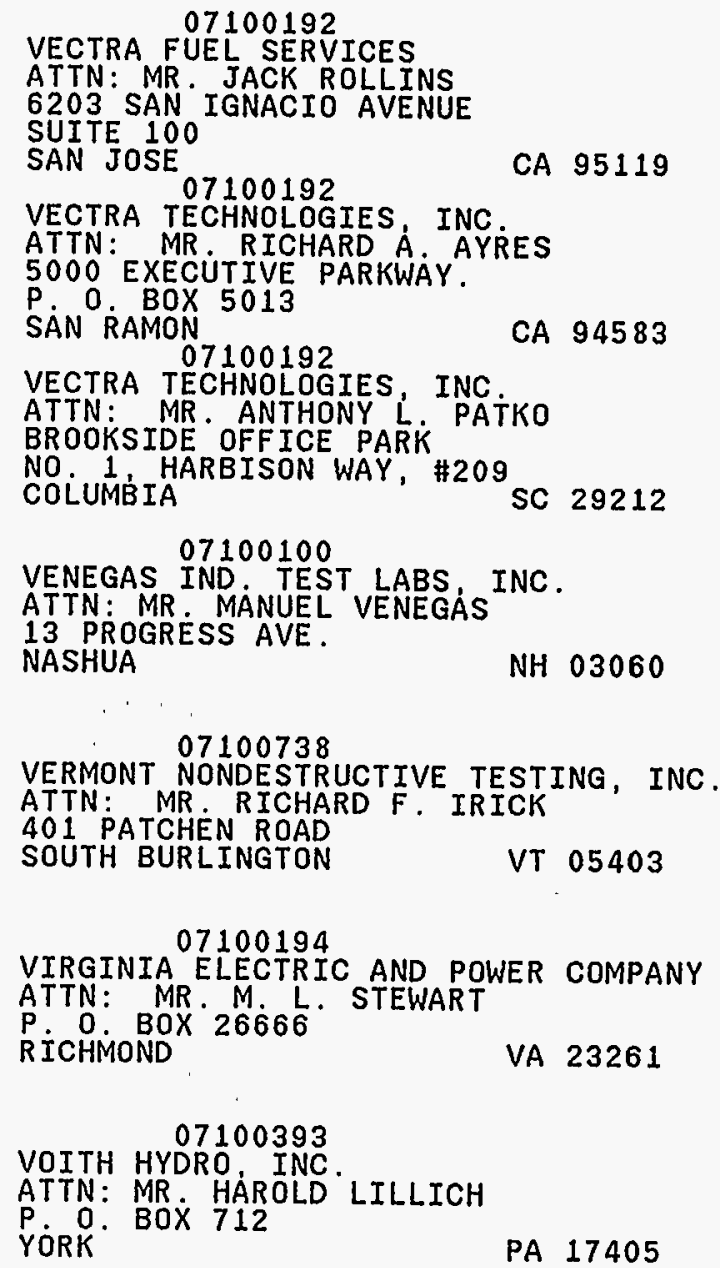

VA 23261

VOITH HYDRO

ATTN: MR. HÁROLD LILLICH

P. O. BOX 712

PA 17405

07100538 
WALASHEK INDUSTRIES

ATTN: MR . CARL K YAMAMOTO

607 ALA MOANA BLVD. PIER

HONOLULU

HI 96813

\section{5}

WASH. PUB. PWR SUPPLY SYSTEM

ATTN: MR $\mathrm{J}_{9} \cdot \mathrm{V}$. PARRISH

3000 GEORGE WASHINGTON WAY

RICHLAND

WA 99352

07100757

WELDTEK TESTING LABS. INC.

ATTN: MR GARY D. CABBLE

2241 HIGHWAY 78 WEST

OXFORD

AL 36203

WELLESLEY 07100845

ATTN: T. KAYE PETERMAN

DEPT. OF BIOLOGICAL SCIENCES

106 CENTRAL STREET

MA 021818283

07100846

WEST VIRGINIA UNIVERSITY HOSPITALS

ATTN : MR. ERIC RAUDENBUSH

MORGANTOWN

WV 265068150

WESTERN 07100780 INDUSTRIAL X-RAY INSPECTION COMPANY, INC

ATTN: MR. LARRY D. WICKS

5354 HIGHWAY 89 NORTH,

EVANSTON

WY 82931

WESTERN $\begin{aligned} & 07100532 \\ & X-R A Y\end{aligned}$

ATTN: MR JAMES E. PETERS

3803 BRANSON ROAD

POTEAU

OK 74953 
QUALITY ASSURANCE APPROVALS BY USER NAME

$U$. S. NUCLEAR REGULATORY COMMISSION

07100765

WESTEX COMPANY

ATTN: MR . KEN GRAYBILL

P. 0 . BOX 5587

CA 93031

07100708

WESTINGHOUSE ELECTRIC CORP., ENERGY SYS. BUS. UN ATTN: MR. A. J. NARDI

PITTSBURGH

PA 15230

07100638

WESTINGHOUSE ELECTRIC CORP., NUCLEAR MFG. DIV.

ATTN: MR. A. J. NARDI

PITTSBURGH

PA 152300355

WILLIAMS 07100847

ATTN: DR ANNE R. SKINNER

DEPARTMENT OF CHEMISTRY

WILLIAMSTOWN

MA 012672692

07100339

WISCONSIN CENTRIFUGAL INC.

AT

SOS EAST ST.

WI 531883898

07100378

WISCONSIN ELECTRIC POWER CO.

ATT: MR C W. FAY

P. 0 BOX 2046

MILWAUKEE

WI 53201

07100262

WISCONSIN INDUSTRIAL TESTING, INC.

ATTN: MR TERRY WALLANDER

BROOKFIELD

WI 53005 


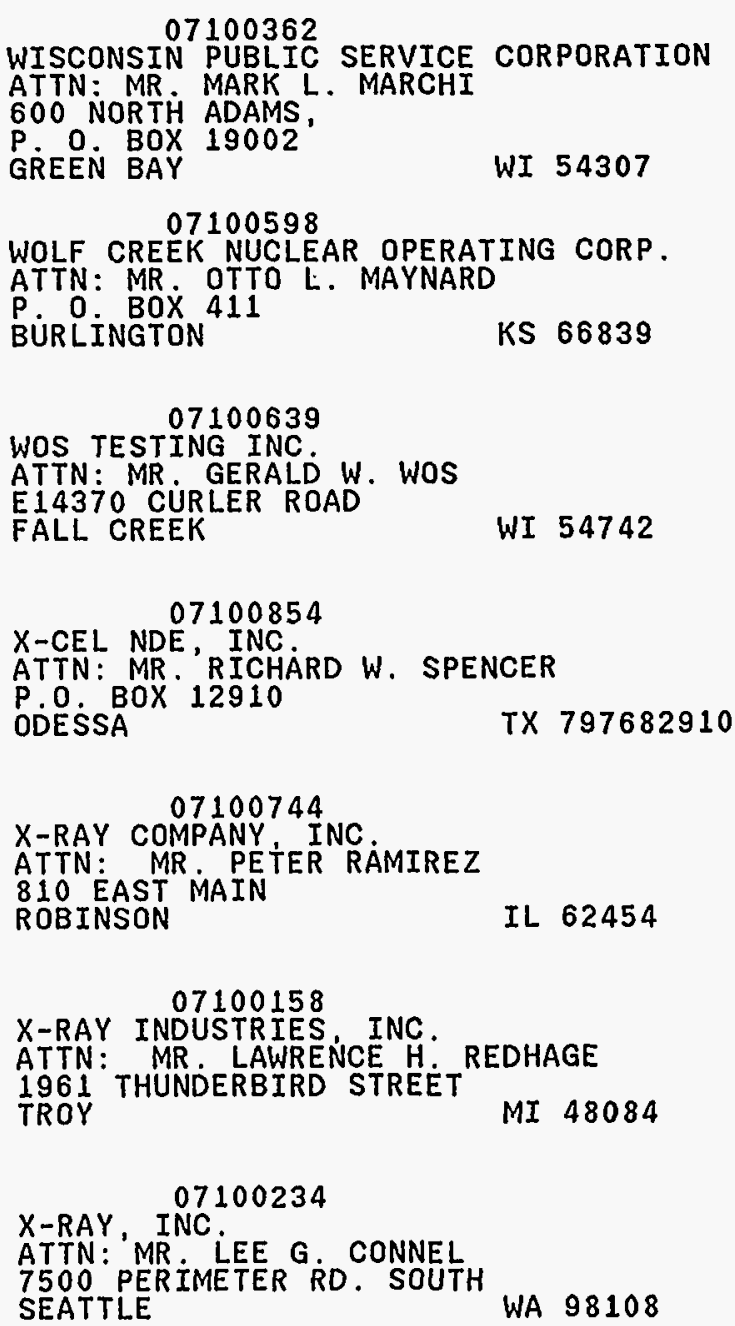

TX 797682910

X-RAY COMPANY 0710074

X-RAY COMPANY INC

810 EAST MAIN

ROBINSON

IL 62454

X-RAY INDUSTO0158

$X$-RAY INDUSTRIES, INC

. REDHAGE

TROY THUNDERBIRD STREET MI 48084

$X$-RAY, INC 07100234

ATTN: 'MR LEE G CONNEL
7500 PERIMETER RO. SOUTH

SEATTLE WA 98108 

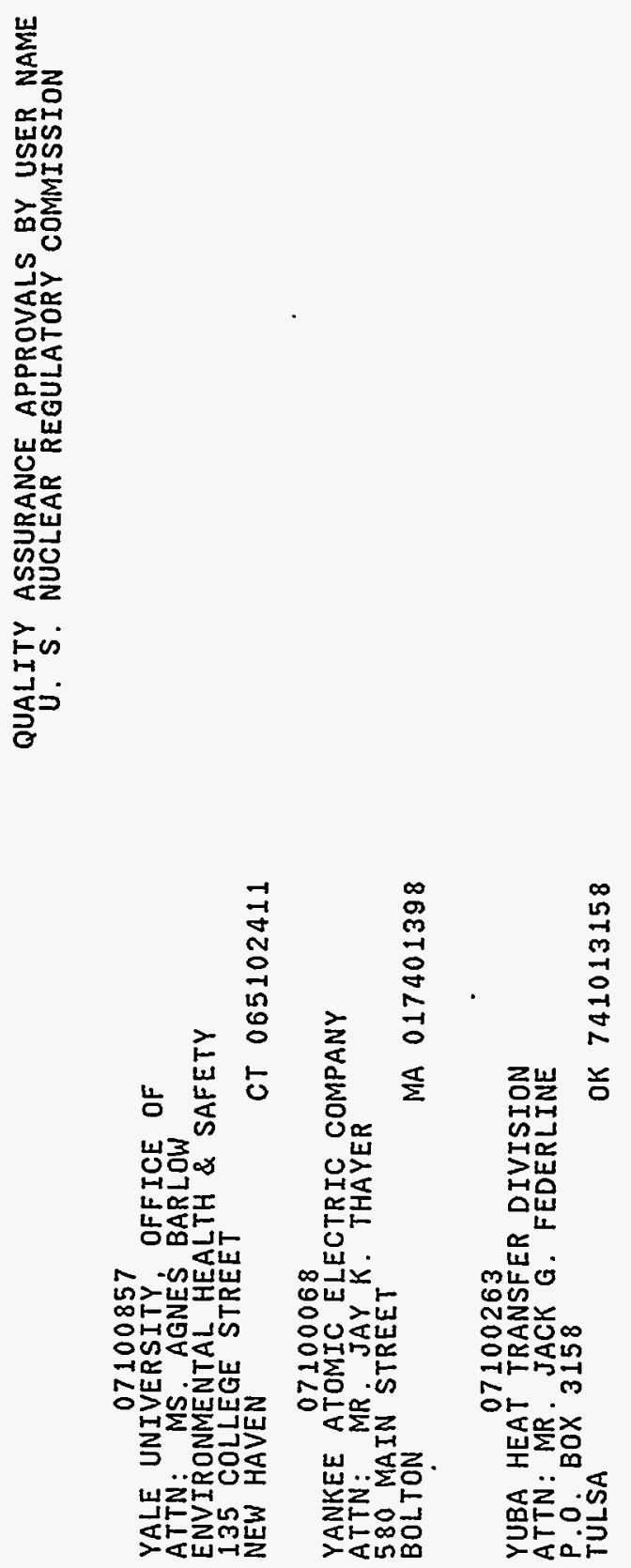


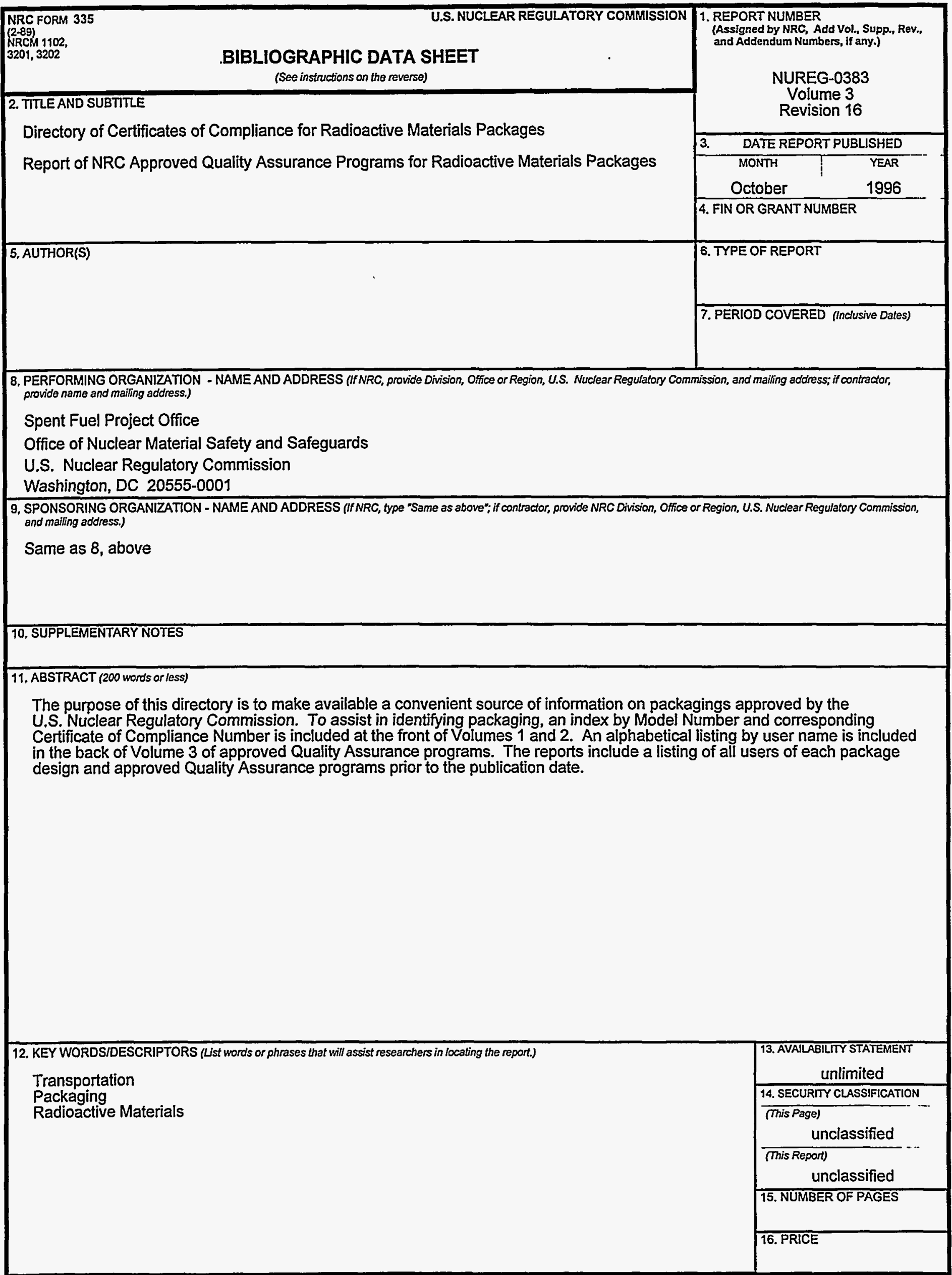




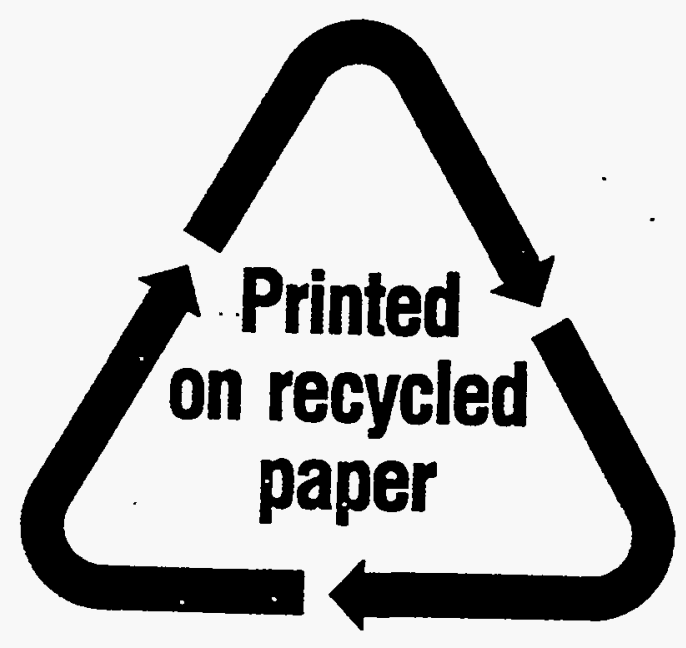

Federal Recycling Program 